JOURNAL OF THE AMERICAN MATHEMATICAL SOCIETY

Volume 20, Number 3, July 2007, Pages 701-751

S 0894-0347(06)00530-3

Article electronically published on April 11, 2006

\title{
UNITARY SHIMURA CORRESPONDENCES FOR SPLIT REAL GROUPS
}

\author{
J. ADAMS, D. BARBASCH, A. PAUL, P. TRAPA, AND D. A. VOGAN, JR.
}

\section{INTRODUCTION}

Let $G$ be the split real form of a connected reductive algebraic group $\mathbb{G}$. Let $\theta$ be a Cartan involution of $G$ and $K=G^{\theta}$ the corresponding maximal compact subgroup. Let $B$ be a Borel subgroup of $G$ with unipotent radical $N$. Then

$$
A=B \cap \theta B
$$

is a $\theta$-stable split Cartan subgroup of $G$. Notice the departure from standard notation for real reductive groups, in which $A$ often refers to the identity component of a maximal split torus. We will write this identity component as $A^{0}$. In our notation, the Iwasawa decomposition is $G=K A^{0} N$. Write

$$
M=B \cap K=A \cap K \simeq \mathbb{Z}_{2}^{n} ;
$$

then

$$
B=A N=M A^{0} N .
$$

Let $\mathfrak{a}=\operatorname{Lie}(A)$. For $\nu \in \mathfrak{a}_{\mathbb{C}}^{*}=\operatorname{Hom}_{\mathbb{R}}(\mathfrak{a}, \mathbb{C})$, the character $e^{\nu}$ of $A^{0}$ extends naturally to a character of $B$ trivial on $M N$. Let

$$
I_{G}(\nu)=\operatorname{Ind}_{B}^{G}\left(e^{\nu}\right)
$$

(normalized induction) be the corresponding induced representation, a (possibly nonunitary) spherical principal series representation. Let $J_{G}(\nu)$ be the irreducible spherical composition factor of $I_{G}(\nu)$, i.e., the unique irreducible composition factor containing the trivial representation $K$. Define the spherical complementary series of $G$ :

$$
\mathbf{C S}(G)=\left\{\nu \in \mathfrak{a}_{\mathbb{C}}^{*} \mid J_{G}(\nu) \text { is unitary }\right\} \subset \mathfrak{a}_{\mathbb{C}}^{*} .
$$

(More precisely, we require that $J_{G}(\nu)$ be infinitesimally equivalent to a unitary representation.) This is a closed, Weyl group-invariant subset of the complex vector space $\mathfrak{a}_{\mathbb{C}}^{*}$.

Received by the editors September 6, 2005.

2000 Mathematics Subject Classification. Primary 22E46.

Key words and phrases. Complementary series.

The first author was supported in part by NSF grant 0532393

The second author was supported in part by NSF grants 0070561 and 0300172 .

The fourth author was supported in part by NSF grant 0300106.

The fifth author was supported in part by NSF grants 9721441 and 0532088 .

This work began during a visit in 2002 to the Institute for Mathematical Sciences, National University of Singapore. The visit was supported by the Institute and the National University of Singapore. We are grateful to our colleagues at NUS for their generous hospitality. 
Apart from the exceptional groups of Type E, $\mathbf{C S}(G)$ has been explicitly computed ([V2], [V3, Ba1], Ci] ).

The goal of this paper is to consider a natural analogue of the spherical complementary series for certain nonlinear groups, and to relate them to spherical complementary series for linear groups. We begin by introducing the nonlinear groups in question.

Definition 1.1. Assume that $\mathbb{G}$ is simple and simply connected. In this case the split real form $G$ is a connected Lie group. It turns out that $G$ admits a unique nontrivial two-fold cover $\widetilde{G}$. That is, we have a short exact sequence of Lie groups

$$
1 \rightarrow\{ \pm 1\} \rightarrow \widetilde{G} \rightarrow G \rightarrow 1
$$

with $\widetilde{G}$ connected. (Such coverings have been studied by many people for a long time. There are general results about coverings of algebraic groups in [St. A detailed description of coverings of real algebraic groups may be found in $[\mathrm{A}$.) The group $\widetilde{G}$ is the nonlinear group we wish to study.

Write $\widetilde{H}$ for the inverse image in $\widetilde{G}$ of a subgroup $H$ of $G$. A representation $\pi$ of $\widetilde{H}$ is called genuine if $\pi(-1)=-I$. If $\pi$ is irreducible, then $\pi$ is genuine if and only if $\pi$ does not factor to $H$.

The subgroups $A^{0}$ and $N$ of $B$ are connected and simply connected and hence lift to subgroups of $\widetilde{G}$. We therefore have a semidirect product decomposition

$$
\widetilde{B}=\widetilde{A} N=\widetilde{M} A^{0} N
$$

with $N$ normal. Fix an irreducible genuine representation $\tilde{\delta}$ of $\widetilde{M}$. We also assume that $\tilde{\delta}$ is pseudospherical (Definition 4.9); this additional assumption is automatic except for $\operatorname{Sp}(2 n, \mathbb{R})$ and $\operatorname{Spin}(2 n+1,2 n)$. For any $\nu \in \mathfrak{a}^{*}, \tilde{\delta} \otimes e^{\nu}$ defines a representation of $\widetilde{B}$. The pseudospherical principal series representation is

$$
I_{\widetilde{G}}(\tilde{\delta}, \nu)=\operatorname{Ind}_{\widetilde{B}}^{\widetilde{G}}\left(\tilde{\delta} \otimes e^{\nu}\right) .
$$

Just as in the spherical case, this principal series representation has a distinguished irreducible composition factor $J_{\widetilde{G}}(\tilde{\delta}, \nu)$ containing a certain $\widetilde{K}$-type (Proposition 5.2). Define the pseudospherical complementary series of $\widetilde{G}$ :

$$
\mathbf{C S}(\tilde{\delta}, \widetilde{G})=\left\{\nu \in \mathfrak{a}_{\mathbb{C}}^{*} \mid J_{\widetilde{G}}(\tilde{\delta}, \nu) \text { is unitary }\right\} .
$$

Again this is a closed Weyl group-invariant subset of the vector space $\mathfrak{a}_{\mathbb{C}}^{*}$. It is independent of the choice of the pseudospherical representation $\tilde{\delta}$ (cf. Lemma 5.4).

We are interested in comparing $\mathbf{C S}(\tilde{\delta}, \widetilde{G})$ to the spherical complementary series of a linear group $G^{\ell}$. For example if $G=\operatorname{SL}(2, \mathbb{R})$, then (if we identify $\mathfrak{a}_{\mathbb{C}}^{*}$ with $\mathbb{C}$ in such a way that the roots are \pm 2 )

$$
\mathbf{C S}(G)=i \mathbb{R} \cup[-1,1] .
$$

Here the imaginary axis corresponds to unitarily induced spherical principal series representations, the open interval $(-1,1)$ to Bargmann's complementary series representations, and the endpoints \pm 1 to the trivial representation. Similarly,

$$
\mathbf{C S}(\tilde{\delta}, \widetilde{G})=i \mathbb{R} \cup[-1 / 2,1 / 2] .
$$

In this case the endpoints correspond to a summand of a metaplectic representation of $\widetilde{\mathrm{SL}}(2, \mathbb{R})$. The two sets $\mathbf{C S}(G)$ and $\mathbf{C S}(\tilde{\delta}, \widetilde{G})$ are identical except for a scale factor of $1 / 2$. 
In order to formulate such a comparison in general, we must first describe the linear group $G^{\ell}$. What we seek to achieve is that representation theory on the nonlinear group $\widetilde{G}$ should be formally similar to representation theory on the linear group $G^{\ell}$. (The most important point will be to relate the reducibility hyperplanes for the pseudospherical principal series $I_{\widetilde{G}}(\tilde{\delta}, \nu)$ to the reducibility hyperplanes of the spherical principal series $I_{G^{\ell}}\left(\nu^{\ell}\right)$.) We therefore refer to $G^{\ell}$ as a linear reincarnation of $\widetilde{G}$. It will be a split real form of a simple algebraic group $\mathbb{G}^{\ell}$, with a split Borel subgroup and maximal torus $\mathbb{B}^{\ell} \supset \mathbb{A}^{\ell}$.

Write $B^{\ell}, A^{\ell}, M^{\ell}$, and $\delta^{\ell}$ (an arbitrary character of $M^{\ell}$ ) for the objects on $G^{\ell}$ corresponding to $B, A, M$, and $\delta$ on $G$. We say that a principal series representation $I_{G^{\ell}}\left(\delta^{\ell}, \nu^{\ell}\right)$ of $G^{\ell}$ is pseudospherical if its restriction to the identity component $\left(G^{\ell}\right)^{0}$ of $G^{\ell}$ is spherical. The corresponding complementary series $\mathbf{C S}\left(\delta^{\ell}, G^{\ell}\right)$ is independent of $\delta^{\ell}$ and is equal to the spherical complementary series $\mathbf{C S}\left(\left(G^{\ell}\right)^{0}\right)$. (This easy fact will be proved in Lemma 4.20.)

The construction of $\mathbb{G}^{\ell}$ will provide a natural covering homomorphism

$$
\Psi: \mathbb{A}^{\ell} \rightarrow \mathbb{A} \text {. }
$$

The differential $d \Psi$ is an isomorphism which carries each coroot of $\mathbb{G}^{\ell}$ to a multiple (usually two) of a coroot of $\mathbb{G}$. In particular, $d \Psi$ identifies the Weyl groups $W$ and $W^{\ell}$.

The group $G^{\ell}$ and the homomorphism $\Psi$ are analogues of the construction of Sa] and Sa2] in the $p$-adic case. See Remark 7.3 .

Here is our main conjecture.

Conjecture 1.3. Suppose $\widetilde{G}$ is a double cover of a split real form of a simply connected complex algebraic group (Definition 1.1). Let $G^{\ell}$ be the split linear group described in Definitions 1.6 and 1.7. Fix a pseudospherical representation $\delta^{\ell}$ of $M^{\ell}$, and a genuine pseudospherical representation $\tilde{\delta}$ of $\widetilde{M}$. Then $d \Psi^{t}$ (cf. (1.2)) induces a bijection

$$
\mathbf{C S}(\tilde{\delta}, \widetilde{G}) \rightarrow \mathbf{C S}\left(\delta^{\ell}, G^{\ell}\right) .
$$

In the case of one root length or $G_{2}, G^{\ell}$ is locally isomorphic to $G$ and $d \Psi$ is multiplication by 2 . If we replace $G^{\ell}$ with $G$ (which does not affect Conjecture 1.3), then the correspondence takes $I_{\widetilde{G}}(\tilde{\delta}, \nu)$ to $I_{G}(2 \nu)$. In this case the conjecture says

$$
J_{\widetilde{G}}(\tilde{\delta}, \nu) \text { is unitary } \Leftrightarrow J_{G}(2 \nu) \text { is unitary } \quad\left(G \text { simply laced or } G_{2}\right) \text {. }
$$

This is known a posteriori for $\mathrm{SL}(n)$ ([Hu1], V2] ) and $G_{2}$ ([V3]); the case of $\mathrm{SL}(2)$ follows from the classical description of complementary series given above. In the case of two root lengths differing by a factor of two, $G^{\ell}$ is locally isomorphic to the dual group of $G$.

Theorem 1.4. If $G$ is a classical group, then $d \Psi^{t}$ induces an injection

$$
\mathbf{C S}(\tilde{\delta}, \widetilde{G}) \hookrightarrow \mathbf{C S}\left(\delta^{\ell}, G^{\ell}\right) .
$$

The case of $G=\operatorname{Sp}(2 n, \mathbb{R})$ is particularly interesting. In this case, $G^{\ell}=$ $\mathrm{SO}(n+1, n) ; \Psi: A^{\ell} \simeq A$ is an isomorphism, given by the identity map in the usual coordinates. (One can find in Chapitre 6 of $\mathrm{Bo}$ ] an embedding of each simple root system in a Euclidean space $\mathbb{R}^{n}$. It is these embeddings that we call "usual coordinates".) The correspondence $J(\tilde{\delta}, \nu) \rightarrow J\left(\nu^{\ell}\right)$ agrees with the Howe dual pair 
correspondence. In this case we can prove Conjecture 1.3, which takes the following form. Recall that the maximal compact subgroup $K$ of $\operatorname{Sp}(2 n, \mathbb{R})$ is $\mathrm{U}(n)$. Its preimage $\widetilde{K}$ in $\widetilde{\mathrm{Sp}}(2 n, \mathbb{R})$ has a genuine character $\operatorname{det}^{1 / 2}$ whose square is the determinant character of $\mathrm{U}(n)$. This character of $\widetilde{K}$ is the representation mentioned in Definition 1.1 as characterizing the distinguished composition factor $J(\tilde{\delta}, \nu)$ of $I(\tilde{\delta}, \nu)$. That is, the irreducible representations of $\widetilde{\mathrm{Sp}}(2 n, \mathbb{R})$ containing $\operatorname{det}^{1 / 2}$ are precisely the various $J(\tilde{\delta}, \nu)$.

Theorem 1.5. Suppose $\pi$ is an irreducible representation of $\widetilde{\operatorname{Sp}}(2 n, \mathbb{R})$ containing the $\widetilde{K}$-type $\operatorname{det}^{1 / 2}$. Let $\theta(\pi)$ denote the representation of $\mathrm{SO}(n+1, n)$ corresponding to $\pi$ via the Howe correspondence. Then $\pi$ is unitary if and only if $\theta(\pi)$ is unitary.

This paper uses many of the ideas of Ba1 and uses the main result of that paper in the proof of Theorem 1.4. The main technique is to reduce the question of unitarity of a representation $\pi$ to the computation of the signature of its Hermitian form restricted to the $K$-isotypic subspaces for certain "petite" representations of $K$ (Definition 4.9). On these $K$-types the Hermitian form is given by a formal computation involving representations of Weyl groups. The resulting Weyl group calculations for $\widetilde{G}$ and $G^{\ell}$ are closely related, and this is enough to give the injection of Theorem 1.4. This is all discussed in Section 13, For $\widetilde{\operatorname{Sp}}(2 n)$ we use the dual pair correspondence to obtain the reverse inclusions (Section 14).

We conclude this introduction with a little more information about the definition of $G^{\ell}$. Here is a precise description in case $G$ is simply laced: that is, in case the roots of $G$ all have the same length. The description applies equally to groups of type $G_{2}$.

Definition 1.6. Suppose $\mathbb{G}$ is simple and simply connected, with maximal torus $\mathbb{A}$ having Lie algebra $\mathfrak{a}$. The cocharacter lattice of $\mathbb{A}$ is

$$
X_{*}(\mathbb{A})=R^{\vee} \subset \mathfrak{a},
$$

the lattice of coroots. The character lattice is

$$
X^{*}(\mathbb{A})=P \subset \mathfrak{a}^{*},
$$

the lattice of integral weights. The root lattice $R$ is a sublattice in $P$ of finite index, and the integral coweight lattice $P^{\vee}$ contains $R^{\vee}$ with finite index.

An algebraic group covered by the simply connected group $\mathbb{G}$ is specified precisely by its cocharacter lattice $L$. This can be any lattice between $R^{\vee}$ and $P^{\vee}$ :

$$
R^{\vee} \subset L \subset P^{\vee} .
$$

When $\mathbb{G}$ is simply laced or of type $G_{2}$, we define $\mathbb{G}^{\ell}$ to be the group covered by $\mathbb{G}$ and having cocharacter lattice

$$
L=\frac{1}{2} R^{\vee} \cap P^{\vee}
$$

Clearly $L$ contains $R^{\vee}$ (since $R^{\vee}$ is contained in both $\frac{1}{2} R^{\vee}$ and $P^{\vee}$ ), and $L$ is contained in $P^{\vee}$. Write $\mathbb{A}^{\ell}$ for the torus of $\mathbb{G}^{\ell}$ with cocharacter lattice $L$. Multiplication by 2 defines a $W$-equivariant inclusion

$$
\Psi: L \rightarrow R^{\vee},
$$

and therefore by functoriality an algebraic map of tori

$$
\Psi: \mathbb{A}^{\ell} \rightarrow \mathbb{A} .
$$


This is the map of (1.2). Write $G^{\ell}$ for a split real form of $\mathbb{G}^{\ell}$, containing the split real form $A^{\ell}$ of $\mathbb{A}^{\ell}$. The mapping $\Psi$ defines

$$
\Psi: A^{\ell} \rightarrow A,
$$

which carries $M^{\ell}$ into $M$ and $\left(A^{\ell}\right)^{0}$ isomorphically onto $A^{0}$, in a $W$-equivariant way. Composition with the differential $d \Psi$ defines a map on characters in the other direction:

$$
d \Psi^{t}: \mathfrak{a}^{*} \rightarrow\left(\mathfrak{a}^{\ell}\right)^{*} .
$$

If we identify $\mathfrak{a}$ with $\mathfrak{a}^{\ell}$ (as we may, since the corresponding groups are locally isomorphic), then $d \Psi^{t}$ is just multiplication by 2 .

In the same way, we have a map on characters

$$
\Psi^{t}:\{\text { characters of } M\} \rightarrow\left\{\text { characters of } M^{\ell}\right\} .
$$

We will see in Lemma 6.4 below that $\Psi^{t}$ induces a natural simply transitive action of the group of pseudospherical characters of $M^{\ell}$ on the set of pseudospherical genuine representations of $\widetilde{M}$. If we fix a single pseudospherical genuine representation of $\widetilde{M}$, this means that we get a bijection

$$
\text { \{genuine pseudosph. chars. of } \widetilde{M}\} \leftrightarrow\left\{\text { pseudosph. chars. of } M^{\ell}\right\} \text {. }
$$

This bijection can be incorporated into Conjecture 1.3 it adds nothing to the difficulty of proving the conjecture, but makes the statement more attractive. (One may hope that the bijection will also be relevant to global statements relating automorphic forms on linear and nonlinear groups.)

The description of $G^{\ell}$ in the remaining cases (Definition 6.1 below) is more complicated; here are some of the main points.

Definition 1.7. Suppose $\mathbb{G}$ is a simply connected simple group not of type $G_{2}$; that is, that any two root lengths differ by a factor of one or two. We will define in Section $\left[\right.$ a connected simple algebraic group $\mathbb{G}^{\ell}$ with root system dual to that of $\mathbb{G}$. We let $G^{\ell}$ be a split real form. Because $G^{\ell}$ has the dual root system to that of $G$, the Weyl groups $W$ and $W^{\ell}$ may be identified. The construction of $\mathbb{G}^{\ell}$ will provide a natural Weyl group-equivariant covering map

$$
\Psi: A^{\ell} \rightarrow A .
$$

Just as in the simply laced case, $\Psi^{t}$ induces a natural simply transitive action (explained in Lemma 6.4) of the group of pseudospherical representations of $M^{\ell}$ on the set of pseudospherical genuine representations of $\widetilde{M}$.

\section{Representations of covers of Abelian groups}

In this section $H$ is an abelian Lie group with finite component group $H / H^{0}$. We consider the representation theory of a (possibly nonabelian) two-fold cover $\widetilde{H}$ of $G$. This means that there is a short exact sequence

$$
1 \rightarrow\{ \pm 1\} \rightarrow \widetilde{H} \stackrel{p}{\rightarrow} H \rightarrow 1 .
$$

A representation $\pi$ of $\widetilde{H}$ is called genuine if $\pi(-1)=-I$. Write $\Pi_{g}(\widetilde{H})$ and $\Pi_{g}(Z(\widetilde{H}))$ for the set of equivalence classes of irreducible genuine representations of $\widetilde{H}$ and $Z(\widetilde{H})$ respectively. 
Proposition 2.2. For every $\chi \in \Pi_{g}(Z(\widetilde{H}))$ there is a unique representation $\pi \in$ $\Pi_{g}(\widetilde{H})$ for which $\left.\pi\right|_{Z(\widetilde{H})}$ is a multiple of $\chi$. Denote this representation by $\pi(\chi)$.

The map $\chi \rightarrow \pi(\chi)$ defines a bijection $\Pi_{g}(Z(\widetilde{H})) \rightarrow \Pi_{g}(\widetilde{H})$. The dimension of $\pi(\chi)$ is $n$, and $\operatorname{Ind}_{Z(\widetilde{H})}^{\widetilde{H}}(\chi)=n \pi$, where $n=|\widetilde{H} / Z(\widetilde{H})|^{\frac{1}{2}}$.

Proof. The key point is that if $\pi$ is a genuine representation of $\widetilde{H}$, then the character $\theta_{\pi}$ of $\pi$ is supported on $Z(\widetilde{H})$, as we now show. Suppose $g \in \widetilde{H}-Z(\widetilde{H})$. Choose $h \in \widetilde{H}$ so that $h g h^{-1} \neq g$. Since $H$ is abelian, $p\left(h g h^{-1}\right)=p(h) p(g) p(h)^{-1}=p(g)$, so $h g h^{-1}=-g$. Computing $\theta_{\pi}$ of both sides we have $\theta_{\pi}\left(h g h^{-1}\right)=\theta_{\pi}(g)=$ $\chi(-1) \theta_{\pi}(g)$. Since $\chi$ is genuine, $\chi(-1)=-1$, so $\theta_{\pi}(g)=0$.

Therefore every irreducible genuine representation of $\widetilde{H}$ is determined by its restriction to $Z(\widetilde{H})$, and any two irreducible representations with the same central character are isomorphic. Fix $\chi \in \Pi_{g}(Z(\widetilde{H}))$. Let $I(\chi)=\operatorname{Ind}_{Z(\widetilde{H})}^{\widetilde{H}}(\chi)$. This has central character $\chi$, so there exists an irreducible representation $\pi(\chi)$, and $I(\chi)=c \pi(\chi)$ for some integer $c$. The dimension of $I(\chi)$ is $|\widetilde{H} / Z(\widetilde{H})|$, so

$$
\left.I(\chi)\right|_{Z(\widetilde{H})}=|\widetilde{H} / Z(\widetilde{H})| \chi, \quad \operatorname{dim} \pi(\chi)=|\widetilde{H} / Z(\widetilde{H})| / c .
$$

By Frobenius reciprocity, it follows that

$$
\operatorname{dim} \operatorname{Hom}_{\widetilde{H}}(I(\chi), I(\chi))=\operatorname{dim} \operatorname{Hom}_{Z(\widetilde{H})}\left(\left.I(\chi)\right|_{Z(\widetilde{H})}, \chi\right)=|\widetilde{H} / Z(\widetilde{H})| .
$$

On the other hand, $I(\chi)$ is a direct sum of $c$ copies of one irreducible representation (the complete reducibility following from finiteness of $H / H^{0}$ ). Schur's lemma therefore implies that

$$
\operatorname{dim} \operatorname{Hom}_{\widetilde{H}}(I(\chi), I(\chi))=c^{2} .
$$

Comparing these two formulas gives $c=n$, and the remaining claims are immediate.

For $x, y \in H$ let $\tilde{x}, \tilde{y}$ be inverse images of $x, y$ in $\widetilde{H}$, and let $\{x, y\}=\tilde{x} \tilde{y} \tilde{x}^{-1} \tilde{y}^{-1}$. This is independent of the choices of $\tilde{x}, \tilde{y}$, and is contained in $\operatorname{ker}(p)=\{ \pm 1\}$. The center of $\widetilde{H}$ is the inverse image of the radical of this form: if we define

$$
Z_{0}(H)=p(Z(\widetilde{H}))
$$

then it follows that

$$
Z_{0}(H)=\{x \in H \mid\{x, y\}=1 \text { for all } y \in H\} .
$$

\section{The CENTER of $\widetilde{A}$}

We return now to the setting of Definition $1.1 \mathbb{G}$ is a connected, simple, simply connected, algebraic group, with split real form $G$ and nontrivial two-fold cover $\widetilde{G}$. The split torus $\widetilde{A}=\widetilde{M} A^{0}$ of $\widetilde{G}$ is a two-fold cover of $A$. To study principal series of $\widetilde{G}$ we need to understand the irreducible representations of $\widetilde{A}$. By Proposition 2.2, we need to compute the center of $\widetilde{A}$. Because the identity component $A^{0}$ of $\widetilde{A}$ is automatically central, it suffices to compute the center of $\widetilde{M}$. By (2.3) we need to compute the radical of the commutator pairing (defined in Section 21).

Let $R^{\vee}$ be the coroot lattice of $\mathbb{A}$, and

$$
P=\operatorname{Hom}_{\mathbb{Z}}\left(R^{\vee}, \mathbb{Z}\right)
$$


the dual lattice of integral weights. Write $\langle$,$\rangle for the canonical pairing P \times R^{\vee} \rightarrow \mathbb{Z}$. Since $\mathbb{G}$ is simply connected, the character and cocharacter lattices of $\mathbb{A}$ are

$$
P=X^{*}(\mathbb{A}), \quad R^{\vee}=X_{*}(\mathbb{A}) .
$$

Consequently

$$
\mathbb{A}=R^{\vee} \otimes_{\mathbb{Z}} \mathbb{C}^{\times}, \quad A=R^{\vee} \otimes_{\mathbb{Z}} \mathbb{R}^{\times}
$$

via the homomorphism $\lambda^{\vee} \otimes x \mapsto \lambda^{\vee}(x)$. In particular, the subgroup $M$ of $A$ (consisting of all elements of order 2) may be identified as

$$
M=R^{\vee} / 2 R^{\vee}
$$

via the homomorphism $\lambda^{\vee} \mapsto \lambda^{\vee}(-1)$.

We recall the definition of the Hilbert symbol over $\mathbb{R}$ : for $x, y \in \mathbb{R}^{\times}$we have

$$
(x, y)_{\mathbb{R}}= \begin{cases}-1 & x<0, y<0, \\ 1 & \text { otherwise }\end{cases}
$$

The rational vector space $R^{\vee} \otimes_{\mathbb{Z}} \mathbb{Q}$ carries a Weyl group-invariant positive definite quadratic form $(\cdot, \cdot)$, unique up to a positive scalar multiple (which we will fix in a moment). By duality this form induces a positive quadratic form (still written $(\cdot, \cdot))$ on $P \otimes_{\mathbb{Z}} \mathbb{Q}$, which contains the roots. In this dual form, the roots have at most two (squared) lengths. Roots of the greater (or only) root length are called $l o n g$, and the other roots (if any) are called short. We normalize the quadratic form so that the greater (or only) root length is 2 :

$$
(\alpha, \alpha)=2 \text { if } \alpha \text { is a long root. }
$$

Because the natural pairing of a root with its coroot is equal to 2 , this requirement is equivalent to

$$
\left(\alpha^{\vee}, \alpha^{\vee}\right)=2 \text { if } \alpha \text { is a long root. }
$$

The other root length is equal to 1 in types $B_{n}, C_{n}$, and $F_{4}$, and equal to $2 / 3$ in type $G_{2}$. Short coroots have (squared) length 2 ; the other coroot length is 6 for $G_{2}$ and 4 in types $B_{n}, C_{n}$, and $F_{4}$.

We emphasize that in the simply laced case, all roots are called long.

With this normalization, the quadratic form takes integer values on the coroot lattice $R^{\vee}$. By $\mathrm{Ma}$, the commutator for $\widetilde{A}$ is given on $A$ by

$$
\left\{\gamma^{\vee} \otimes x, \lambda^{\vee} \otimes y\right\}=(x, y)_{\mathbb{R}}^{\left(\gamma^{\vee}, \lambda^{\vee}\right)} \quad\left(\gamma^{\vee}, \lambda^{\vee} \in R^{\vee} ; x, y \in \mathbb{R}^{\times}\right) .
$$

The quadratic form $($,$) is positive definite, and in particular nondegenerate. It$ therefore induces an isomorphism (from left to right)

$$
R^{\vee} \otimes \mathbb{Q} \simeq \operatorname{Hom}_{\mathbb{Q}}\left(R^{\vee} \otimes \mathbb{Q}, \mathbb{Q}\right) .
$$

Since $P \simeq \operatorname{Hom}_{\mathbb{Z}}\left(R^{\vee}, \mathbb{Z}\right)$, we obtain an isomorphism (in the opposite direction)

$$
\Gamma: P \otimes \mathbb{Q} \simeq R^{\vee} \otimes \mathbb{Q} .
$$

Tracing through the definitions we see that

$$
\left\langle\lambda, \gamma^{\vee}\right\rangle=\left(\Gamma(\lambda), \gamma^{\vee}\right) \quad\left(\lambda \in P, \gamma^{\vee} \in R^{\vee}\right) .
$$

In particular we conclude

$$
\Gamma(P)=\left\{\gamma^{\vee} \in R^{\vee} \otimes \mathbb{Q} \mid\left(\gamma^{\vee}, \alpha^{\vee}\right) \in \mathbb{Z} \text { for all } \alpha^{\vee} \in R^{\vee}\right\} .
$$


Except in the case of $C_{n}$ or $G_{2}, \Gamma$ is the identity in the usual coordinates ([Bo], Chapitre 6 ). For $C_{n}$ (respectively $G_{2}$ ) we have $\Gamma=1 / 2($ respectively $1 / 3$ ).

Let

$$
c=\max _{\alpha, \beta} \frac{(\alpha, \alpha)}{(\beta, \beta)} \in\{1,2,3\} .
$$

Let $t(\alpha)=1$ if $\alpha$ is long, and $c$ if $\alpha$ is short.

Lemma 3.5. For all roots $\alpha$,

$$
\begin{aligned}
\Gamma(\alpha) & =\frac{1}{t(\alpha)} \alpha^{\vee} \\
& =\frac{2 \alpha^{\vee}}{\left(\alpha^{\vee}, \alpha^{\vee}\right)} .
\end{aligned}
$$

Proof. Fix a long root $\beta$ so that $\left\|\beta^{\vee}\right\|^{2}=2$. That $\Gamma$ commutes with the simple reflection $s_{\alpha}$ is equivalent to $\Gamma(\alpha)=\tau(\alpha) \alpha$ for some $\tau(\alpha) \in \mathbb{Q}$. By $(3.4)(\mathrm{b})$ we have

$$
\begin{aligned}
2 & =\left\langle\alpha, \alpha^{\vee}\right\rangle=\left(\tau(\alpha) \alpha^{\vee}, \alpha^{\vee}\right) \\
& =\tau(\alpha)\left(\left\|\alpha^{\vee}\right\|^{2} /\left\|\beta^{\vee}\right\|^{2}\right)\left\|\beta^{\vee}\right\|^{2} \\
& =2 \tau(\alpha)\|\beta\|^{2} /\|\alpha\|^{2},
\end{aligned}
$$

so $\tau(\alpha)=\|\alpha\|^{2} /\|\beta\|^{2}$. For the final equality note that

$$
\|\alpha\|^{2} /\|\beta\|^{2}=\left\|\beta^{\vee}\right\|^{2} /\left\|\alpha^{\vee}\right\|^{2}=2 /\left\|\alpha^{\vee}\right\|^{2} .
$$

From 3.4)(b and Lemma 3.5 we have the following useful formulas (for $\alpha$ any root):

$$
\begin{aligned}
t(\alpha)\langle\alpha, \Gamma(\lambda)\rangle & =\left\langle\lambda, \alpha^{\vee}\right\rangle \quad\left(\lambda \in P \otimes_{\mathbb{Z}} \mathbb{Q}\right), \\
t(\alpha)\left\langle\alpha, \gamma^{\vee}\right\rangle & =\left(\alpha^{\vee}, \gamma^{\vee}\right) \quad\left(\gamma^{\vee} \in R^{\vee}\right) .
\end{aligned}
$$

Note that 3.6)(a) implies that for $\lambda \in P \otimes_{\mathbb{Z}} Q,\langle\alpha, \Gamma(\lambda)\rangle \in \mathbb{Z}$ if and only if $\frac{1}{t(\alpha)}\left\langle\lambda, \alpha^{\vee}\right\rangle \in \mathbb{Z}$. In particular if $\mathbb{G}$ is simply laced we conclude

$$
\Gamma(R)=R^{\vee}, \quad \Gamma(P)=P^{\vee} .
$$

Another convenient formula for the commutator is Lemma 4.8 below.

The following lemma is similar to [Sa, Section 2.2] and [Sa2, page 117].

Lemma 3.8. In the identification (3.2) of $M$ with $R^{\vee} / 2 R^{\vee}$, the preimage of the center of $\widetilde{M}$ is

$$
Z_{0}(M) \simeq\left[2 \Gamma(P) \cap R^{\vee}\right] / 2 R^{\vee} .
$$

Proof. By (3.3) the commutator on $M$ is given by

$$
\left\{\gamma^{\vee}(-1), \lambda^{\vee}(-1)\right\}=(-1)^{\left(\gamma^{\vee}, \lambda^{\vee}\right)} \quad\left(\gamma^{\vee}, \lambda^{\vee} \in R^{\vee}\right) .
$$

By (3.4)(c) we conclude that $\gamma^{\vee}(-1) \in Z_{0}(M) \Leftrightarrow \gamma^{\vee} \in 2 \Gamma(P)$, which gives (3.9).

It is sometimes convenient to use the following commutator formula.

Lemma 3.10. Suppose $g \in A, \alpha^{\vee}$ is a coroot, and $x \in \mathbb{R}^{\times}$. Then

$$
\left\{g, \alpha^{\vee}(x)\right\}=(\alpha(g), x)_{\mathbb{R}}^{t(\alpha)},
$$

where $t(\alpha)=1$ if $\alpha$ is long, and $c$ if it is short. 
Proof. It is enough to check this for $g=\beta^{\vee}(y)$ for $\beta^{\vee} \in R^{\vee}$. The left-hand side is then $(y, x)_{\mathbb{R}}^{\left(\beta^{\vee}, \alpha^{\vee}\right)}$. The right-hand side is

$$
\left(\alpha\left(\beta^{\vee}(y)\right), x\right)_{\mathbb{R}}^{t(\alpha)}=\left(y^{\left\langle\alpha, \beta^{\vee}\right\rangle}, x\right)_{\mathbb{R}}^{t(\alpha)}=(x, y)_{\mathbb{R}}^{t(\alpha)\left\langle\alpha, \beta^{\vee}\right\rangle} .
$$

The result follows from 3.6$)(\mathrm{b})$.

In the simply laced case the result is particularly simple.

Lemma 3.12. If $G$ is simply laced or of type $G_{2}$, then $Z(\widetilde{M})=Z(\widetilde{G})$ and $Z(\tilde{A})=$ $Z(\tilde{G}) A^{0}$.

Proof. The hypothesis means that $t(\alpha)$ is always odd, and therefore it can be dropped from (3.11) (since the values of the Hilbert symbol are \pm 1 ). It follows immediately that $Z_{0}(M)=\{m \in M \mid \alpha(m)=1$ for all $\alpha\}=Z(G)$. Similarly $Z_{0}(A)=\{g \in A \mid \alpha(g)>0$ for all $\alpha\}$. It is easy to see this is precisely $Z(G) A^{0}$.

Using Lemmas 3.8 and 3.12, it is easy to compute $Z_{0}(M)$ in each case. Its cardinality (equal to the number of irreducible genuine representations of $\widetilde{M}$ ) is given in Table 1 in Section 6 .

Example 3.13. Let $G=\operatorname{Spin}(n, n)$ or $\operatorname{Spin}(n+1, n)$. In the usual coordinates $P=\mathbb{Z}^{n} \cup\left(\mathbb{Z}+\frac{1}{2}\right)^{n}$ and $R^{\vee}=\mathbb{Z}_{e}^{n}$ (elements of $\mathbb{Z}^{n}$ for which the sum of the coordinates is even). We conclude

$$
2 \Gamma(P) \cap R^{\vee}= \begin{cases}2 \mathbb{Z}^{n} \cup(2 \mathbb{Z}+1)^{n} & n \text { even, } \\ 2 \mathbb{Z}^{n} & n \text { odd, }\end{cases}
$$

and $\left|Z_{0}(M)\right|=4$ or 2 respectively.

Let $\lambda^{\vee}=(2,0, \ldots, 0)$ in $R^{\vee}$, and $\gamma_{ \pm}^{\vee}=(1,1, \ldots, \pm 1)$ (which is in $R^{\vee}$ if $n$ is even). Then $Z_{0}(M)$ contains $\lambda^{\vee}(-1)$, and also $\gamma_{ \pm}^{\vee}(-1)$ if $n$ is even.

Example 3.14. Let $G$ be the split group of type $F_{4}$. Then in the usual coordinates, representatives for $2 \Gamma(P) \cap R^{\vee} / 2 R^{\vee}$ are $0,(2,0,0,0),( \pm 1,1,1,1)$.

\section{PSEudospherical PRINCIPAL SERIES}

In this section we recall some additional structure for split real groups. In order to treat at the same time the nonlinear group $\widetilde{G}$ of Definition 1.1 and the linear group $G^{\ell}$ (whose definition will be completed in Section 6), it is convenient for this section to weaken our assumptions on $G$ and to work in the following setting.

Definition 4.1. Assume that $G$ is a split real form of a connected reductive algebraic group, with Cartan involution $\theta$, Borel subgroup $B$, and split maximal torus

$$
A=B \cap \theta B \subset B .
$$

Assume that $\widetilde{G}$ is a (possibly trivial) two-fold cover of $G$; that is, that we have a short exact sequence of Lie groups

$$
1 \rightarrow\{ \pm 1\} \rightarrow \widetilde{G} \rightarrow G \rightarrow 1 .
$$

In general we write $\widetilde{H}$ for the preimage in $\widetilde{G}$ of any subgroup $H$ of $G$. A representation $\pi$ of $\widetilde{H}$ is called genuine if $\pi(-1)=-I$. Write $N$ for the unipotent radical 
of $B$ and $A^{0}$ for the identity component of $A$. Because these groups are simply connected, their coverings must split. We identify

$$
N=\text { identity component of } \widetilde{N} \text {, }
$$

and similarly for $A^{0}$.

If we choose $\widetilde{G}$ to be the trivial cover $G \times\{ \pm 1\}$, then genuine representations of $\widetilde{G}$ may be identified with representations of $G$. Therefore a discussion of "genuine representations of $\widetilde{G}$ " in the setting of Definition 4.1 covers both split linear groups and the nonlinear groups of Definition 1.1 .

Let $N(\widetilde{A})$ be the normalizer of $\widetilde{A}$ in $\widetilde{G}$, and let $W=N(\widetilde{A}) / \widetilde{A}$ (the Weyl group of $\widetilde{A}$ in $\widetilde{G}$ ). This group is independent of the covering and may be identified (through its action on the Lie algebra $\mathfrak{a}$ ) with the Weyl group of the root system of $G$. Because $\widetilde{A}$ may be nonabelian, the action of $N(\widetilde{A})$ on $\widetilde{A}$ may not factor to an action of $W$; but we do obtain an action of $W$ on $Z(\widetilde{A})$. In any case $N(\widetilde{A})$ acts on $\Pi_{g}(\widetilde{A})$ (the set of genuine irreducible representations of $\widetilde{A}$ ) by

$$
(g \cdot \pi)(a)=\pi\left(g^{-1} a g\right) \quad\left(\pi \in \Pi_{g}(\widetilde{A}), g \in N(\widetilde{A}), a \in \widetilde{A}\right) .
$$

This factors to an action of $W$ on $\Pi_{g}(\widetilde{A})$ in all cases: if $g \in \widetilde{A}$, then $\pi \simeq g \cdot \pi$, with intertwining operator $\pi\left(g^{-1}\right)$. For $w \in W$ and $\chi$ a genuine character of $Z(\widetilde{A})$ we have (in the notation of Proposition 2.2)

$$
\pi(\chi)^{w} \simeq \pi\left(\chi^{w}\right)
$$

Corresponding statements hold with $\widetilde{M}$ in place of $\widetilde{A}$.

We denote (real) Lie algebras by the corresponding Gothic letters $\mathfrak{g}, \mathfrak{k}, \mathfrak{a}$, etc.

Let $\alpha$ be a root of $\mathfrak{a}$ in $\mathfrak{g}$. Choose a Lie algebra homomorphism

$$
\phi_{\alpha}: \mathfrak{s l}(2, \mathbb{R}) \longrightarrow \mathfrak{g}
$$

and define

$$
Z_{\alpha}=\phi_{\alpha}\left(\begin{array}{cc}
0 & 1 \\
-1 & 0
\end{array}\right) \in \mathfrak{k}
$$

as in [V4, 4.3.6]. (This means that $\phi_{\alpha}$ carries the diagonal torus in $\mathfrak{s l}(2, \mathbb{R})$ into $\mathfrak{a}$, the upper triangular matrices into the root space $\mathfrak{g}_{\alpha}$, and $\mathfrak{s o}(2)$ into $\mathfrak{k}$. In particular, $\phi_{\alpha}$ intertwines the negative transpose automorphism of $\mathfrak{s l}(2, \mathbb{R})$ with the Cartan involution of $\mathfrak{g}$.) The homomorphism $\phi_{\alpha}$ is unique up to conjugation of $\mathfrak{s l}(2, \mathbb{R})$ by the diagonal matrix with entries $i$ and $-i$. As a consequence, the Lie algebra element $Z_{\alpha}$ is defined only up to sign.

Exponentiating we obtain homomorphisms

$$
\widetilde{\Phi}_{\alpha}: \widetilde{\mathrm{SL}}(2, \mathbb{R}) \rightarrow \widetilde{G}, \quad \Phi_{\alpha}: \operatorname{SL}(2, \mathbb{R}) \rightarrow G .
$$

Here $\widetilde{\mathrm{SL}}(2, \mathbb{R})$ is the unique nontrivial two-fold cover of $\operatorname{SL}(2, \mathbb{R})$. If $\widetilde{G}$ is linear, then $\widetilde{\Phi}_{\alpha}$ factors through $\operatorname{SL}(2, \mathbb{R})$; otherwise this may or may not be the case.

Definition 4.4. A root $\alpha$ is said to be metaplectic if $\widetilde{\Phi}_{\alpha}$ does not factor to $\operatorname{SL}(2, \mathbb{R})$.

Lemma 4.5 (for example, $\underline{\mathrm{A}}$, Theorem 1.6]). Suppose $\widetilde{G}$ is the nontrivial double cover of the group of real points of a simply connected simple split group. If $G$ is not of type $G_{2}$, then $\alpha$ is metaplectic if and only if $\alpha$ is long. If $G$ is of type $G_{2}$, then all roots are metaplectic. 
Let

$$
\widetilde{m}_{\alpha}=\exp _{\widetilde{G}}\left(\pi Z_{\alpha}\right) \in \widetilde{M} .
$$

Changing the choice of $\phi_{\alpha}$ replaces $Z_{\alpha}$ by $-Z_{\alpha}$, and so replaces $\widetilde{m}_{\alpha}$ by $\widetilde{m}_{\alpha}^{-1}$. The image of $\widetilde{m}_{\alpha}$ in $G$ is

$$
m_{\alpha}=\exp _{G}\left(\pi Z_{\alpha}\right) \in M .
$$

If $\alpha$ is not metaplectic - for example if $\widetilde{G}$ is linear - then $\widetilde{m}_{\alpha}$ has order 2 and is independent of the choice of $\Phi_{\alpha}$, as in [V4, 4.3.6]. Almost as the definition of the coroot $\alpha^{\vee}$, we have

$$
\Phi_{\alpha}\left(\begin{array}{cc}
t & 0 \\
0 & t^{-1}
\end{array}\right)=\alpha^{\vee}(t)
$$

In particular,

$$
m_{\alpha}=\alpha^{\vee}(-1) \in A \text {. }
$$

If $\alpha$ is metaplectic, then $\widetilde{m}_{\alpha}$ does depend on the choice of $\Phi_{\alpha}$; changing the choice replaces $\widetilde{m}_{\alpha}$ by $\widetilde{m}_{\alpha}^{-1}$. More explicitly, recall that $\{ \pm 1\}$ is the kernel of the covering map from $\widetilde{G}$ to the linear group $G$. Then $\widetilde{m}_{\alpha}$ has order $4, \widetilde{m}_{\alpha}^{2}=-1$ and $\widetilde{m}_{\alpha}^{-1}=-\widetilde{m}_{\alpha}$. Let

$$
h_{1}=\exp \left(\begin{array}{cc}
0 & \pi \\
-\pi & 0
\end{array}\right), \quad h_{2}=\exp \left(\begin{array}{cc}
0 & -\pi \\
\pi & 0
\end{array}\right)
$$

be the two elements of $\widetilde{\mathrm{SL}}(2, \mathbb{R})$ lying over $-I \in \mathrm{SL}(2, \mathbb{R})$. Then

$$
\widetilde{m}_{\alpha}=\widetilde{\Phi}_{\alpha}\left(h_{1}\right) \text {. }
$$

We have $h_{i}^{4}=1$ and $h_{2}=h_{1}^{-1}$. (4.7).

The following formula is an immediate consequence of (3.3), Lemma 3.5 and

Lemma 4.8. Suppose $\widetilde{G}$ is the nontrivial double cover of the group of real points of a simply connected simple split group. For all roots $\alpha, \beta$,

$$
\left\{m_{\alpha}, m_{\beta}\right\}= \begin{cases}(-1)^{\left\langle\alpha, \beta^{\vee}\right\rangle} & \alpha, \beta \text { metaplectic }, \\ 1 & \text { otherwise. }\end{cases}
$$

Definition 4.9. Suppose $\widetilde{G}$ is a split group as in Definition 4.1.

(1) An irreducible representation $\tilde{\delta}$ of $\widetilde{M}$ is pseudospherical if -1 is not an eigenvalue of $\tilde{\delta}\left(\widetilde{m}_{\alpha}\right)$ for any $\alpha$.

(2) An irreducible representation $\mu$ of $\widetilde{K}$ is

$$
\begin{cases}\text { pseudospherical } & \\
\text { fine } & \text { if }|\gamma| \leq\left\{\begin{array}{l}
\frac{1}{2} \\
1 \\
\text { petite }
\end{array}\right.\end{cases}
$$

respectively, for every root $\alpha$ and every eigenvalue $\gamma$ of $d \mu\left(i Z_{\alpha}\right)$. More generally, we say that a $K$-type $\mu$ is level $k$ if $|\gamma| \leq k$ for every $\alpha$ and every eigenvalue $\gamma$ of $d \mu\left(i Z_{\alpha}\right)$.

(3) A representation of $\widetilde{G}$ is pseudospherical if it contains a pseudospherical $\widetilde{K}$-type. 
Because $Z_{\alpha}$ is defined using a generator for the Lie algebra of $\mathrm{SO}(2)$ or its double cover, every eigenvalue $\gamma$ of $d \mu\left(i Z_{\alpha}\right)$ belongs to $\frac{1}{2} \mathbb{Z}$ (if $\alpha$ is metaplectic) or to $\mathbb{Z}$ (if not).

Remark 4.10. Suppose $\tilde{\delta}$ is genuine. If $\alpha$ is metaplectic, then condition (1) in Definition 4.9 is automatic: $\widetilde{m}_{\alpha}^{2}=-1$ and the only possible eigenvalues of $\tilde{\delta}\left(\widetilde{m}_{\alpha}\right)$ are $\pm i$, independent of the choice of $\widetilde{m}_{\alpha}$. In particular if $\widetilde{G}$ is simply laced or of type $G_{2}$, then every irreducible genuine representation of $\widetilde{M}$ is pseudospherical.

Suppose $\alpha$ is not metaplectic. Because $\widetilde{m}_{\alpha}^{2}=1, \tilde{\delta}\left(\widetilde{m}_{\alpha}\right)$ has eigenvalues \pm 1 . Note that the inverse image in $\widetilde{G}$ of $m_{\alpha} \in G$ consists of $\pm \widetilde{m}_{\alpha}$. If $\tilde{\delta}$ is genuine, then $\tilde{\delta}\left(-\widetilde{m}_{\alpha}\right)=-\tilde{\delta}\left(\widetilde{m}_{\alpha}\right)$. Thus the definition of pseudospherical depends on the fact that we have specified $\widetilde{m}_{\alpha}$ to be the unique inverse image of $m_{\alpha}$ that is in the image of $\Phi_{\alpha}$.

Lemma 4.11. Suppose that $\widetilde{G}$ is as in Definition 4.1, and $\tilde{\delta}$ is an irreducible genuine representation of $\widetilde{M}$.

(1) Suppose $\beta$ is a root of $A$ in $G$ that is not metaplectic (Definition 4.4), so that $\widetilde{\Phi}_{\beta}: \mathrm{SL}(2, \mathbb{R}) \rightarrow \widetilde{G}$. Then the element $\widetilde{m}_{\beta}$ of (4.6) has order at most 2 , and belongs to $Z(\widetilde{M})$.

(2) The representation $\tilde{\delta}$ is pseudospherical if and only if $\tilde{\delta}\left(\widetilde{m}_{\beta}\right)=1$ for all nonmetaplectic roots $\beta$. This is a condition on the central character $\left.\tilde{\delta}\right|_{Z(\widetilde{M})}$.

(3) The action of the Weyl group $W$ on irreducible genuine representations of $\widetilde{M}$ fixes each pseudospherical representation.

(4) Define $Z_{0}(M)$ to be the preimage in $M$ of $Z(\widetilde{M})$, and $\bar{Z}_{0}(M)$ its quotient by the subgroup generated by elements $m_{\beta}$ for $\beta$ not metaplectic. Then there is a natural simply transitive action of the group of characters of $\bar{Z}_{0}(M)$ on the genuine pseudospherical representations of $\widetilde{M}$. The character $\bar{\chi}$ acts on $\tilde{\delta}$ by tensoring the central character of $\tilde{\delta}$ with the pullback of $\bar{\chi}$ to $Z(\widetilde{M})$. Equivalently, one can choose any extension of $\bar{\chi}$ to a character $\chi$ of $M$, and tensor $\tilde{\delta}$ with the pullback of $\chi$ to $\widetilde{M}$.

Proof. In (1), that $\widetilde{m}_{\beta}$ has order at most 2 is immediate from the definition of metaplectic (see (4.6) ). If $\widetilde{m} \in \widetilde{M}$, then

$$
\operatorname{Ad}(\widetilde{m})\left(Z_{\alpha}\right)=\alpha(\widetilde{m}) Z_{\alpha}= \pm Z_{\alpha} .
$$

Multiplying by $\pi$ and exponentiating gives

$$
\widetilde{m} \widetilde{m}_{\alpha} \widetilde{m}^{-1}=\widetilde{m}_{\alpha}^{\alpha(\widetilde{m})} .
$$

If $\widetilde{m}_{\beta}$ has order at most 2 , then this reads

$$
\widetilde{m} \widetilde{m}_{\beta} \widetilde{m}^{-1}=\widetilde{m}_{\beta}^{ \pm 1}=\widetilde{m}_{\beta}
$$

proving that $\widetilde{m}_{\beta}$ is central in $\widetilde{M}$.

For (2), Remark 4.10 shows that the condition of being pseudospherical depends only on the nonmetaplectic roots $\beta$; the rest of the statement follows from (1).

For (3), suppose that $\chi \in \Pi_{g}(Z(\widetilde{M}))$ is the central character of a pseudospherical representation of $\widetilde{M}$. By Proposition 2.2 , it is enough to show that $s_{\alpha} \chi=\chi$ for every simple reflection $s_{\alpha} \in W$. To see this, define

$$
\widetilde{\sigma}_{\alpha}=\exp _{\widetilde{G}}\left(\pi Z_{\alpha} / 2\right) \text {. }
$$


The image of $\widetilde{\sigma}_{\alpha}$ in $G$ is

$$
\sigma_{\alpha}=\exp _{G}\left(\pi Z_{\alpha} / 2\right)=\Phi_{\alpha}\left(\begin{array}{cc}
0 & 1 \\
-1 & 0
\end{array}\right)
$$

a representative for the reflection $s_{\alpha}$ in the Weyl group. We wish to show that

$$
\chi\left(\widetilde{\sigma}_{\alpha}^{-1} \widetilde{m} \widetilde{\sigma}_{\alpha}\right)=\chi(\widetilde{m})
$$

for all $\widetilde{m} \in Z(\widetilde{M})$.

Multiplying (4.12) by $\pi / 2$ and exponentiating gives

$$
\widetilde{m} \widetilde{\sigma}_{\alpha} \widetilde{m}^{-1}=\widetilde{\sigma}_{\alpha}^{\alpha(\widetilde{m})}= \begin{cases}\widetilde{\sigma}_{\alpha} & \text { if } \alpha(\widetilde{m})=1, \\ \widetilde{\sigma}_{\alpha} \widetilde{m}_{\alpha}^{-1} & \text { if } \alpha(\widetilde{m})=-1 .\end{cases}
$$

We multiply this equation by $\widetilde{\sigma}_{\alpha}^{-1}$ on the left and by $\widetilde{m}$ on the right, obtaining

$$
\widetilde{\sigma}_{\alpha}^{-1} \widetilde{m} \widetilde{\sigma}_{\alpha}= \begin{cases}\widetilde{m} & \text { if } \alpha(\widetilde{m})=1, \\ \widetilde{m}_{\alpha}^{-1} \widetilde{m} & \text { if } \alpha(\widetilde{m})=-1 .\end{cases}
$$

The left side of (4.15) is therefore

$$
\chi\left(\widetilde{\sigma}_{\alpha}^{-1} \widetilde{m} \widetilde{\sigma}_{\alpha}\right)= \begin{cases}\chi(\widetilde{m}) & \text { if } \alpha(\widetilde{m})=1, \\ \chi\left(\widetilde{m}_{\alpha}^{-1} \widetilde{m}\right) & \text { if } \alpha(\widetilde{m})=-1 .\end{cases}
$$

We now consider two possibilities. If $\alpha$ is not metaplectic, then $\chi\left(\widetilde{m}_{\alpha}\right)=1$ by $(2)$, so both cases of (4.16) are equal to $\chi(\widetilde{m})$, as we wished to show.

If $\alpha$ is metaplectic, then $\widetilde{m}_{\alpha}^{-1}=-\widetilde{m}_{\alpha}$ (see the discussion after (4.7)). Now (4.13) and the assumption that $\widetilde{m}$ is central in $\widetilde{M}$ imply that $\alpha(\widetilde{m})=1$. We are therefore in the first case of (4.16), so we have $\chi(\widetilde{m})$, as we wished to show.

Part (4) follows from the description of genuine representations of $\widetilde{M}$ in Proposition 2.2

Remark 4.17. For a discussion of fine $K$-types for linear groups see [V4, 4.3]. Petite $K$-types are discussed in Section 12 .

Here is a characterization of petite representations of $\widetilde{K}$ in terms of their highest weights. Because the group $\widetilde{K}$ may be disconnected, a little care is required in talking about highest weights. If $\widetilde{G}$ is nonlinear, then $\widetilde{K}$ is connected, and the group $T_{K}$ in the following lemma is a maximal torus. If $\widetilde{G}$ is linear, then its Cartan subgroups are abelian; so $T_{K}$ is an abelian subgroup of $K$ containing a maximal torus. We write $X^{*}\left(T_{K}\right)$ for the abelian group of characters of $T_{K}$; this maps by restriction onto the lattice $X^{*}\left(T_{K}^{0}\right)$ of characters of the maximal torus. Each imaginary root $\beta$ gives rise to a three-dimensional root subalgebra of $\mathfrak{g}$, which is isomorphic either to $\mathfrak{s l}(2, \mathbb{R})$ (if $\beta$ is noncompact) or to $\mathfrak{s u}(2)$ (if $\beta$ is compact). These isomorphisms $\psi_{\beta}$ may be chosen to satisfy

$$
\psi_{\beta}(\mathfrak{s o}(2)) \subset \mathfrak{t}_{K}
$$

in both cases, and we may calculate the coroot as

$$
\beta^{\vee}=\psi_{\beta}\left(\begin{array}{cc}
0 & i \\
-i & 0
\end{array}\right) \in i \mathfrak{t}_{K} .
$$


If $\beta$ is noncompact, the map $\psi_{\beta}$ may exponentiate only to the double cover $\widetilde{\mathrm{SL}}(2, \mathbb{R})$, and so the coroot $\beta^{\vee}$ may take half-integer values on the differential of a character of $T_{K}$. This explains how half integers can arise in the lemma.

Lemma 4.18. Suppose $\widetilde{G}$ is a split group as in Definition 4.1, and suppose $\mu$ is an irreducible representation of $\widetilde{K}$. Choose a fundamental $\theta$-stable Cartan subgroup $T_{f}$ of $\widetilde{G}$. Thus $T_{K}=T_{f} \cap \widetilde{K}$ contains a maximal torus of $\widetilde{K}$. Let $\lambda \in X^{*}\left(T_{K}\right)$ be a highest weight of $\mu$ (with respect to some choice of positive roots). If $G$ is of type $B_{2 n+1}$ or $G_{2}$, let

$$
C=\left\{\text { noncompact imaginary roots } \beta \text { of } T_{f} \text { in } G\right\} .
$$

Otherwise let

$C=\left\{\right.$ imaginary roots $\beta$ of $T_{f}$ in $G \mid \beta$ is noncompact long, or compact short $\}$.

If $\beta \in C$, then $\left\langle\lambda, \beta^{\vee}\right\rangle \in \frac{1}{2} \mathbb{Z}$ is defined. The representation $\mu$ is level $k$ (Definition 4.9) if and only if

$$
\left\langle\lambda, \beta^{\vee}\right\rangle \leq k \quad \text { for all } \beta \in C .
$$

Proof. If $\alpha$ is a root of $\mathfrak{a}$, then $Z_{\alpha} \in \mathfrak{t}_{K}$ for some fundamental Cartan subalgebra $\mathfrak{t}_{f}$. The definition of level is in terms of the eigenvalues of the elements $i Z_{\alpha}$; so we need to identify these elements in terms of the coroots of imaginary roots. This can be done using the theory of Cayley transforms ( $[\mathrm{Sc}$, Proposition 2.16; [Kn], pages 417-420; V4, 4.3.6]).

A Cayley transform $c$ from $\mathfrak{a}$ to $\mathfrak{t}_{f}$ may be defined by a maximal set $S$ of strongly orthogonal roots of $\mathfrak{a}$. For each root $\alpha$ in this set, the definition of $c$ identifies $i Z_{\alpha}$ with a noncompact imaginary coroot (of the same length). If $\alpha$ is a long root of $\mathfrak{a}$, then we may choose $S$ containing $\alpha$, and it follows that $i Z_{\alpha}$ is conjugate to the coroot for a long noncompact imaginary root. If $\alpha$ is short the same argument applies in type $B_{2 n+1}$ or $G_{2}$.

The remaining cases are groups of type $B_{2 n}, C_{n}$, or $F_{4}$, in which a maximal strongly orthogonal set of roots must consist entirely of long roots. In this case one can also construct $\mathfrak{t}_{f}$ from $\mathfrak{a}$ using a different maximal set $S^{\prime}$ of orthogonal roots, including exactly two short roots $\alpha$ and $\alpha^{\prime}$. In this case, a calculation in type $B_{2}$ shows that $i Z_{\alpha}$ is conjugate to the coroot corresponding to a short compact imaginary root.

So we may suppose that $i Z_{\alpha}$ is equal to an imaginary coroot $\beta^{\vee}$. The eigenvalue of $i Z_{\alpha}$ on the $\gamma$-weight space of $\mu$ is $\left\langle\gamma, \beta^{\vee}\right\rangle$. If in addition $\beta$ is $K$-dominant (as we may as well assume, since $S$ is invariant under the compact Weyl group), then $\left\langle\gamma, \beta^{\vee}\right\rangle \leq\left\langle\lambda, \beta^{\vee}\right\rangle$. The result follows.

Lemma 4.19. Suppose $G$ is a split linear group as in Definition 4.1. Let $\delta$ be a character of $M$, and let $\mu$ be an irreducible representation of $K$.

(1) The character $\delta$ is pseudospherical if and only if $\delta\left(m_{\alpha}\right)=1$ for every root $\alpha$ (cf. (4.6) ); equivalently, if and only if $\delta$ restricted to $M \cap G^{0}$ is trivial.

(2) The representation $\mu$ is pseudospherical if and only if its restriction to $K \cap$ $G^{0}=K^{0}$ is trivial.

(3) The natural surjections

$$
M \rightarrow G / G^{0}, \quad K \rightarrow G / G^{0}
$$

identify the characters of the component group of $G$ with the pseudospherical representations of $M$ and $K$. 
Proof. The first condition in (1) is clear from the definition of pseudospherical; or it is a special case of Lemma 4.11. Since $M \cap G^{0}$ is generated by the elements $\left\{m_{\alpha}\right\}$, the second condition in (1) follows. Computing in $\mathrm{SO}(2)$ (mapped into $K$ by the homomorphism $\Phi_{\alpha}$ of (4.30), we see that $d \mu\left(i Z_{\alpha}\right)$ can have only integer eigenvalues. Therefore $\mu$ pseudospherical implies $d \mu\left(i Z_{\alpha}\right)=0$. The root space decomposition of $\mathfrak{g}$ shows that the various $\left\{Z_{\alpha}\right\}$ span the Lie algebra of $K$, and (2) follows. Statement (3) is an elementary consequence of (1) and (2).

From the point of view of unitarity, studying pseudospherical representations of a linear group is equivalent to studying spherical representations, as the next lemma shows. We have included pseudospherical (and not just spherical) representations of linear groups primarily because of the bijection of Lemma 6.4.

Lemma 4.20. (1) Suppose $G$ is a Type I group, and $H \subset G$ is a subgroup of finite index. Then an irreducible representation $\pi$ of $G$ is unitary if and only if any, equivalently every, summand of $\pi$ restricted to $H$ is unitary.

(2) Suppose $G$ is a split linear group. Then for all pseudospherical characters $\delta$ of $M$ and $\nu \in \mathfrak{a}^{*},\left.I_{G}(\delta, \nu)\right|_{G^{0}} \simeq I_{G^{0}}(\nu)$, and $\left.J_{G}(\delta, \nu)\right|_{G^{0}} \simeq J_{G^{0}}(\nu)$. In particular, $\mathbf{C S}(\delta, G)=\mathbf{C S}\left(G^{0}\right)$ (notation as in Definition 1.1).

(3) Suppose $G, G^{\prime}$ are split linear groups with a finite number of connected components, which are locally isomorphic, and $\delta, \delta^{\prime}$ are pseudospherical. Then (if we identify $\mathfrak{a}$ and $\mathfrak{a}^{\prime}$ by the local isomorphism) $\mathbf{C S}(\delta, G)=\mathbf{C S}\left(\delta^{\prime}, G^{\prime}\right)$.

Proof. Statement (1) is elementary, and (2) follows from the fact that $G=G^{0} A$. A spherical representation of $G$ factors to $G / Z$ for any finite subgroup $Z$ of the center of $G$, since $Z$ is contained in $K$, and (3) follows easily.

A description of the pseudospherical representations for certain disconnected groups is found in Table 3. The pseudospherical representations of nonlinear groups will be discussed in Section 5

\section{Pseudospherical RePresentations of NONLINEAR Groups}

In this section $\mathbb{G}$ is simple, simply connected, with split real form $G$, and $\widetilde{G}$ is the nontrivial two-fold cover of $G$. Our goal is to describe precisely the pseudospherical representations of $\widetilde{M}$ and of $\widetilde{K}$. More general results (applicable to arbitrary coverings of arbitrary real reductive groups) may be found in WW, 11.A.2]. In our special setting it is possible to get some additional explicit information about the representations, which we will use in the proof of Theorem 1.4. For this reason we give proofs of the results.

We first compute the pseudospherical representations of $\widetilde{M}$. Recall (Proposition 2.2) that the irreducible genuine representations of $\widetilde{M}$ are parametrized by genuine characters of $Z(\widetilde{M})$.

Lemma 5.1. (1) Suppose $G \nsucceq \operatorname{Spin}(2 n+1,2 n)$ or $\operatorname{Sp}(4 n, \mathbb{R})$. Restriction to $Z(\widetilde{G})$ defines a canonical bijection between the genuine pseudospherical representations of $\widetilde{M}$ and the genuine characters of $Z(\widetilde{G})$.

(2) If $G=\operatorname{Spin}(2 n+1,2 n)$ or $\operatorname{Sp}(4 n, \mathbb{R})$, then $\widetilde{M}$ has exactly two genuine pseudospherical representations. These have the same restriction to $Z(\widetilde{G})$.

The pseudospherical representations of $\widetilde{M}$ are listed in Table 1 . 
Proof. Suppose $G$ is simply laced or of type $G_{2}$. Then every root is metaplectic, and therefore (Remark 4.10) every irreducible genuine representation of $\widetilde{M}$ is pseudospherical. On the other hand, $Z(\widetilde{G})=Z(\widetilde{M})$ in this case (Lemma 3.12), and the result follows.

The case of $\operatorname{Sp}(2 n, \mathbb{R})$ is well-known; see Section 12.2. There are two pseudospherical one-dimensional representations $\operatorname{det}^{ \pm 1 / 2}$ of $\widetilde{K}$, whose restrictions $\tilde{\delta}_{ \pm}$are the pseudospherical representations of $\widetilde{M}$. These satisfy $\tilde{\delta}_{+}^{*} \simeq \tilde{\delta}_{-}$. If $n$ is odd, then $Z(\widetilde{G}) \simeq \mathbb{Z} / 4 \mathbb{Z}$ and $\tilde{\delta}_{ \pm}$have distinct characters when restricted to $Z(\widetilde{G})$. If $n$ is even, then $Z(\widetilde{G}) \simeq \mathbb{Z} / 2 \mathbb{Z} \times \mathbb{Z} / 2 \mathbb{Z}$ and $\tilde{\delta}_{ \pm}$have the same central character.

Let $G=\operatorname{Spin}(n+1, n), n \geq 2$. See Example 3.13. For all short roots $\alpha, m_{\alpha}$ is the unique nontrivial central element of $G$. Because $\alpha$ is not metaplectic, it follows that

$$
Z(\widetilde{G})=\left\{1,-1, \widetilde{m}_{\alpha},-\widetilde{m}_{\alpha}\right\} \simeq(\mathbb{Z} / 2 Z)^{2} .
$$

Therefore every genuine pseudospherical representation of $\widetilde{M}$ has the same character when restricted to $Z(\widetilde{G})$ (trivial on $\widetilde{m}_{\alpha}$, and -1 on -1 ); in fact a genuine representation of $\widetilde{M}$ is pseudospherical if and only if it has this restriction. Let $\chi$ be a character of $Z_{0}(M)$ such that $\chi\left(m_{\alpha}\right)=-1$; regard $\chi$ as a (nongenuine) character of $Z(\widetilde{M})$. Then for any genuine character $\widetilde{\xi}$ of $Z(\widetilde{M})$, precisely one of $\widetilde{\xi}$ and $\chi \otimes \widetilde{\xi}$ is pseudospherical. From Table 1 we conclude that $\widetilde{G}$ has two genuine pseudospherical representations if $n$ is even, and one if $n$ is odd. The result follows in this case.

Suppose $G$ is of type $F_{4}$. In this case $Z(G)$ is trivial and $Z_{0}(M)$ is generated by $\left\{m_{\alpha} \mid \alpha\right.$ short $\}$ (cf. Example 3.14$)$. It follows that $Z(\widetilde{M})$ is an elementary two-group and has exactly one genuine pseudospherical representation.

Proposition 5.2. Let $\left(\tilde{\delta}, F_{\tilde{\delta}}\right)$ be a pseudospherical representation of $\widetilde{M}$. There is a unique pseudospherical representation $\left(\mu(\tilde{\delta}), F_{\tilde{\delta}}\right)$ of $\widetilde{K}$ such that $\left.\mu(\tilde{\delta})\right|_{\widetilde{M}}=\tilde{\delta}$. The map $\tilde{\delta} \rightarrow \mu(\tilde{\delta})$ is a bijection between the pseudospherical representations of $\widetilde{M}$ and $\widetilde{K}$.

Proof. Essentially this may be found in Section 6 of V0, but we repeat the argument for completeness. If $\tilde{\delta}$ is not genuine, this is Lemma 4.19] so assume $\tilde{\delta}$ is genuine.

Fix a root $\alpha$. Since $\widetilde{m}_{\alpha}^{4}=1$, the possible eigenvalues of $\tilde{\delta}\left(\widetilde{m}_{\alpha}\right)$ are $\{ \pm 1, \pm i\}$. Since $\tilde{\delta}$ is pseudospherical, -1 does not occur. Therefore $F_{\tilde{\delta}}$ is the sum of the three eigenspaces $F_{\tilde{\delta}}(\alpha, 1)$ and $F_{\tilde{\delta}}(\alpha, \pm i)$. Let $\log$ be the principal branch of the logarithm; recall that

$$
\log (1)=0, \quad \log (i)=i \pi / 2, \quad \log (-i)=-i \pi / 2 .
$$

Recall (4.6) that $\widetilde{m}_{\alpha}=\exp \left(\pi Z_{\alpha}\right)$, so (if $\mu$ exists) $d \mu\left(Z_{\alpha}\right)$ must preserve the $\gamma$ eigenspace $F_{\tilde{\delta}}(\alpha, \gamma)$, and must act there with eigenvalues $\lambda \in \frac{1}{\pi} \log (\gamma)+2 i \mathbb{Z}$. The requirement that $\mu$ be pseudospherical means that $|i \lambda| \leq 1 / 2$. This means that we are forced to define

$$
d \mu\left(Z_{\alpha}\right) v=\frac{\log (\gamma)}{\pi} v \quad\left(v \in F_{\tilde{\delta}}(\alpha, \gamma)\right) .
$$

A little more informally,

$$
d \mu\left(Z_{\alpha}\right)=\frac{\log \left(\tilde{\delta}\left(\widetilde{m}_{\alpha}\right)\right)}{\pi}
$$


This defines $d \mu\left(Z_{\alpha}\right) \in \operatorname{Aut}\left(F_{\tilde{\delta}}\right)$. Note that the eigenvalues of $d \mu\left(i Z_{\alpha}\right)$ are equal to 0 or $\pm 1 / 2$, so $\mu$ is pseudospherical if it is a representation.

The root space decomposition of $\mathfrak{g}$ shows that the $Z_{\alpha}$ are a basis of $\mathfrak{k}$, so $d \mu$ is a well-defined map $\mathfrak{k} \rightarrow \operatorname{Aut}\left(F_{\tilde{\delta}}\right)$.

Uniqueness of $\mu$ is now clear: if $\mu$ is pseudospherical, this is the only possible choice of $d \mu$. For existence, we must show first that the linear map $d \mu$ is a representation of $\mathfrak{k}$. That is, for any two roots $\alpha$ and $\beta$, we must show that

$$
d \mu\left(\left[Z_{\alpha}, Z_{\beta}\right]\right)=\left[d \mu\left(Z_{\alpha}\right), d \mu\left(Z_{\beta}\right)\right] .
$$

This reduces to a rank two calculation, as follows. Let $H$ be the subgroup of $G$ generated by the root subgroups for $\pm \alpha$ and $\pm \beta$, and $\widetilde{H}$ its inverse image in $\widetilde{G}$. The pseudospherical representation $\tilde{\delta}$ of $\widetilde{M}$ is the sum of irreducible genuine pseudospherical representation $\tilde{\delta}_{H}$ of $\widetilde{M}_{H}=\widetilde{M} \cap \widetilde{H}$. If we can prove our claim for $\widetilde{H}$ - that is, show that each $\tilde{\delta}_{H}$ defines a representation $d \mu_{H}$ of $\mathfrak{h} \cap \mathfrak{k}$ by the formula (5.3) - then the commutation relation we want for $Z_{\alpha}$ and $Z_{\beta}$ follows. Evidently $H$ is a connected split semisimple group of type $A_{1} \times A_{1}, A_{2}, B_{2}$, or $G_{2}$. In the first case the claim is obvious. In the remaining cases, the assumption that $G$ is simply connected implies that $H$ must also be simply connected; so $H$ is $\mathrm{SL}(3, \mathbb{R})$, $\operatorname{Sp}(4, \mathbb{R})$, or the split real form of $G_{2}$.

If the covering $\widetilde{H}$ is trivial, then none of the roots of $H$ can be metaplectic, so $\tilde{\delta}_{H}$ is trivial on all $\widetilde{m}_{\gamma}$. It follows that $d \mu_{H}$ is trivial on $\mathfrak{h} \cap \mathfrak{k}$, and in particular $d \mu_{H}$ is a representation.

We may therefore assume that the covering $\widetilde{H}$ is not trivial; so it is the covering of Definition 1.1. We need to consider each genuine pseudospherical representation $\tilde{\delta}_{H}$ of $\widetilde{M}_{H}$ and show that $d \mu_{H}$ is a Lie algebra representation. If $\mu^{\prime}$ is any irreducible genuine pseudospherical representation of $\widetilde{K}_{H}=\widetilde{H} \cap \widetilde{K}$, then the arguments above show that $\mu^{\prime}$ restricts to an irreducible genuine pseudospherical representation $\delta^{\prime}$ of $\widetilde{M}_{H}$, and that $d \mu^{\prime}$ is the linear map defined by $\delta^{\prime}$. This establishes our claim for all such $\delta^{\prime}$. It therefore suffices to show that the number of irreducible genuine pseudospherical representations of $\widetilde{K}_{H}$ is equal to the number of irreducible genuine pseudospherical representations of $\widetilde{M}_{H}$. These numbers (one, two, and one in the cases $A_{2}, B_{2}$, and $G_{2}$ ) may be found in Tables 1 and 2. (They are computed using Lemma 4.18 and Lemma 6.3 below.)

This proves that $d \mu$ is indeed a representation of the Lie algebra $\mathfrak{k}$. To check that $d \mu$ exponentiates to the group $\widetilde{K}$, the simplest approach is to use the fact that $\widetilde{K}$ is simply connected except in type $C_{n}$. We will give a different argument, which can be generalized to show (for any reductive group $G$ ) that "(k, $M)$-modules" satisfying some simple compatibility conditions must always exponentiate to $K$.

It is enough to restrict attention to a maximal torus in $\widetilde{K}$. We can construct such a torus using a maximal strongly orthogonal set of roots $\alpha_{1}, \ldots, \alpha_{n}$. These roots define by (4.3) a group homomorphism

$$
\widetilde{\Phi}=\widetilde{\Phi}_{\alpha_{1}} \times \cdots \times \widetilde{\Phi}_{\alpha_{n}}: \widetilde{\mathrm{SO}}(2)^{n} \rightarrow \widetilde{K} .
$$

The group

$$
\widetilde{T}=\widetilde{\Phi}\left(\widetilde{\mathrm{SO}}(2)^{n}\right)
$$

is a maximal torus in $\widetilde{K}$. By construction (because the eigenvalues involved were half integers) it is clear that $d \mu$ exponentiates to a representation $\mu_{\mathrm{SO}}$ of $\widetilde{\mathrm{SO}}(2)^{n}$. 
Write $x_{i}$ for an element of order 4 in $\widetilde{\mathrm{SO}}(2)_{i}$; that is,

$$
\widetilde{\Phi}\left(x_{i}\right)=\widetilde{m}_{\alpha_{i}} .
$$

Because $\alpha_{i}$ is a well-defined character of $\widetilde{T}$, corresponding to the character 2 of $\mathrm{SO}(2)_{i}$, it follows that

$$
\operatorname{ker} \widetilde{\Phi}=Q \subset\left\langle x_{i} \mid 1 \leq i \leq n\right\rangle=(\mathbb{Z} / 4 \mathbb{Z})^{n} .
$$

In order to show that $\mu_{\widetilde{\mathrm{SO}}}$ descends to $\widetilde{T}$, we must show that it is trivial on $Q$. But we also have a short exact sequence

$$
1 \rightarrow Q \rightarrow\left\langle x_{i}\right\rangle \rightarrow\left\langle\tilde{m}_{\alpha_{i}}\right\rangle \rightarrow 1 .
$$

Since $\mu_{\widetilde{\mathrm{SO}}}\left(x_{i}\right)=\tilde{\delta}\left(\widetilde{m}_{\alpha_{i}}\right)$, it follows that $\mu_{\widetilde{\mathrm{SO}}}$ descends to $\tilde{\delta}$ on $\left\langle\widetilde{m}_{\alpha_{i}}\right\rangle$, and therefore must be trivial on $Q$. Therefore $\mu$ exponentiates to $\widetilde{T}$ and consequently to $\widetilde{K}$.

Suppose $g \in \mathbb{G}(\mathbb{C})$ normalizes $G$. Because the covering $\widetilde{G}$ is canonical, the action of $g$ on $G$ lifts uniquely to an action on $\widetilde{G}$.

Lemma 5.4. $\operatorname{Norm}_{\mathbb{G}(\mathbb{C})}(G)$ acts transitively on the irreducible genuine pseudospherical representations of $\widetilde{M}$ and $\widetilde{K}$.

Proof. The simplest proof is to check in each case that $\operatorname{Norm}_{\mathbb{G}(\mathbb{C})}(G)$ acts transitively on the irreducible genuine pseudospherical $\widetilde{K}$-types (cf. Table 2). For example if $G=\mathrm{SL}(2 n)$, then the action of the element $\operatorname{diag}(-\zeta, \zeta, \ldots, \zeta)$, where $\zeta^{2 n}=-1$,

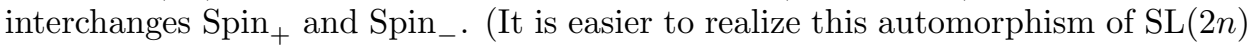
as conjugation by $\operatorname{diag}(-1,1, \ldots, 1) \in \mathrm{GL}(2 n)$.) It is helpful to keep in mind that the map $\mu \rightarrow \mu^{*}$ (sending a representation of $\widetilde{K}$ to its dual) may be realized as the action of an element of $\mathbb{G}(\mathbb{C})$.

The significance of this result is that it implies that $\mathbf{C S}(\tilde{\delta}, \widetilde{G})$, for $\tilde{\delta}$ pseudospherical, is independent of $\tilde{\delta}$. Compare Lemma 4.20,

Finally we can complete the definition of the representations $J(\tilde{\delta}, \nu)$ appearing in the introduction.

Definition 5.5. Suppose $\tilde{\delta}$ is an irreducible genuine pseudospherical representation of $\widetilde{M}$. Let $\mu(\tilde{\delta})$ be the irreducible representation of $\widetilde{K}$ given by Lemma 4.19 and Proposition 5.2. Notice that Frobenius reciprocity implies that $\mu(\tilde{\delta})$ occurs with multiplicity one in $I(\tilde{\delta}, \nu)$. We let $J(\tilde{\delta}, \nu)$ be the unique irreducible constituent of $I(\tilde{\delta}, \nu)$ containing $\mu(\tilde{\delta})$.

It can be shown (compare $[\mathrm{V} 4,6.6 .15])$ that $J(\tilde{\delta}, \nu)$ coincides with the (unique) Langlands subquotient of $I(\tilde{\delta}, \nu)$.

As a consequence of Proposition 5.2, Lemma 4.11, and Harish-Chandra's subquotient theorem, the representation $J(\tilde{\delta}, \nu)$ is the unique irreducible representation of $\widetilde{G}$ containing the $K$-type $\mu(\tilde{\delta})$ and having infinitesimal character $\nu$. We will use this observation to calculate theta correspondences in Section 14.

The following proposition summarizes the conclusions of this section.

Proposition 5.6. There are canonical bijections between the following sets:

(1) irreducible genuine pseudospherical representations of $\widetilde{M}$;

(2) irreducible genuine pseudospherical representations of $\widetilde{K}$; and 
(3) genuine pseudospherical principal series representations of $\widetilde{G}$ with fixed infinitesimal character.

If $G \not 千 \operatorname{Sp}(4 n, \mathbb{R})$ or $\operatorname{Spin}(2 n+1,2 n)(n \geq 1)$, then these sets are also in canonical bijection with

(4) genuine characters of $Z(\widetilde{G})$.

\section{The Group $G^{\ell}$}

We continue with the notation of the preceding section.

Definition 6.1. In order to describe $G^{\ell}$, we begin with its root system $\Delta^{\ell}$ :

$$
\Delta^{\ell}= \begin{cases}\Delta & G \text { simply laced or type } G_{2}, \\ \Delta^{\vee} & G \text { not of type } G_{2} .\end{cases}
$$

Of course the simply laced groups fall into both cases; but then $\Delta$ is naturally isomorphic to $\Delta^{\vee}$ by the map $\Gamma$ (cf. (3.7)). Notice that there is a natural bijection $\alpha \leftrightarrow \alpha^{\ell}$ between $\Delta$ and $\Delta^{\ell}$, defined by

$$
\alpha^{\ell}= \begin{cases}\alpha & G \text { simply laced or type } G_{2}, \\ \alpha^{\vee} & G \text { not of type } G_{2} .\end{cases}
$$

This bijection provides an identification of Weyl groups

$$
W(\Delta) \simeq W\left(\Delta^{\ell}\right), \quad s_{\alpha} \leftrightarrow s_{\alpha^{\ell}} .
$$

Write

$$
R^{\ell} \subset P^{\ell} \subset\left(\mathfrak{a}^{\ell}\right)^{*}
$$

for the root lattice, integral weight lattice, and ambient vector space of $\Delta^{\ell}$; similarly we write

$$
\left(R^{\ell}\right)^{\vee} \subset\left(P^{\ell}\right)^{\vee} \subset \mathfrak{a}^{\ell}
$$

for the coroot and integral coweight lattices.

To complete the definition of $G^{\ell}$, we must describe its coweight lattice $L$,

$$
\left(R^{\ell}\right)^{\vee} \subset L \subset\left(P^{\ell}\right)^{\vee} \text {. }
$$

The definition is

$$
L= \begin{cases}\left\{\gamma \in P^{\vee} \mid 2 \gamma \in R^{\vee}\right\} & G \text { simply laced or type } G_{2}, \\ \left\{\gamma \in P \mid 2 \Gamma(\gamma) \in R^{\vee}\right\} & G \text { not of type } G_{2} .\end{cases}
$$

Notice first of all that $\left(P^{\ell}\right)^{\vee}$ is equal to $P^{\vee}$ in the first case and to $P$ in the second case, so $L$ is actually a sublattice of $\left(P^{\ell}\right)^{\vee}$. Next, the two definitions agree in the simply laced case, since we are then using $\Gamma$ to identify $P$ with $P^{\vee}$. Finally, the fact that $L \supset\left(R^{\ell}\right)^{\vee}$ is obvious in the first case and is a consequence of Lemma 3.5 in the second.

We can now define a linear reincarnation of $\widetilde{G}$ as a complex simple algebraic group $\mathbb{G}^{\ell}$ with root system $\Delta^{\ell}$ and coweight lattice $L$. There is in particular a maximal torus

$$
\mathbb{A}^{\ell}=\mathbb{C}^{\times} \otimes_{\mathbb{Z}} L .
$$

We fix a split real form $G^{\ell}$ of $\mathbb{G}^{\ell}$ with split maximal torus

$$
A^{\ell}=\mathbb{R}^{\times} \otimes_{Z} L .
$$


Define a $W$-equivariant lattice homomorphism

$$
\Psi: L \rightarrow R^{\vee}
$$

by

$$
\Psi(\gamma)= \begin{cases}2 \gamma & G \text { simply laced or type } G_{2}, \\ 2 \Gamma(\gamma) & G \text { not of type } G_{2} .\end{cases}
$$

On the level of coroots, we can calculate from Lemma 3.5 that

$$
\Psi\left(\left(\alpha^{\ell}\right)^{\vee}\right)= \begin{cases}2 \alpha^{\vee} & \text { if } \alpha \text { is metaplectic, } \\ \alpha^{\vee} & \text { if } \alpha \text { is not metaplectic. }\end{cases}
$$

This map induces by functoriality a $W$-equivariant group homomorphism

$$
\Psi: A^{\ell} \rightarrow A
$$

Lemma 6.3. The image of the group homomorphism

$$
\Psi: A^{\ell} \rightarrow A
$$

(Definition 6.1) is equal to $Z_{0}(A)$. The map $\Psi$ restricts to a surjection

$$
\Psi: M^{\ell} \rightarrow Z_{0}(M) .
$$

Proof. Exactly as in (3.2), we have $M^{\ell}=L / 2 L$. It follows that the image $\Psi\left(M^{\ell}\right)$ is

$$
2 L / 2 R^{\vee} \subset R^{\vee} / 2 R^{\vee}=M .
$$

The second assertion is now just a reformulation of Lemma 3.8 if $G$ is not of type $G_{2}$. When $G$ is of type $G_{2}, L=R^{\vee}, \Psi$ is squaring, and the image $\Psi\left(M^{\ell}\right)$ is trivial. Since $Z_{0}(M)$ is also trivial in this case (Lemma 3.12 ), the second assertion is proved in general. The first is an immediate consequence.

Choose a maximal compact subgroup $K^{\ell}$ of $G^{\ell}$, so that $A^{\ell}=M^{\ell}\left(A^{\ell}\right)^{0}$ with $M^{\ell}=A^{\ell} \cap K^{\ell}$ as usual. We count the pseudospherical representations of $M^{\ell}$ (and therefore of $K^{\ell}$ and of the component group of $G^{\ell}$, by Lemma 4.19) in Table 3 .

Lemma 6.4. (1) Suppose that $G$ is simply laced or of type $G_{2}$. Then the kernel of the group homomorphism

$$
\Psi: M^{\ell} \rightarrow Z_{0}(M)
$$

(Lemma 6.3) is the subgroup generated by the various $m_{\alpha^{\ell}}$ (defined in (4.6)); equivalently, it is the intersection $M^{\ell} \cap\left(G^{\ell}\right)^{0}$. Consequently the transpose map

$$
\Psi^{t}:\left\{\text { characters of } Z_{0}(M)\right\} \hookrightarrow\left\{\text { characters of } M^{\ell}\right\}
$$

has as image precisely the pseudospherical characters of $M^{\ell}$ (Definition 4.9).

(2) Suppose that $G$ is not of type $G_{2}$. Then the kernel of the group homomorphism

$$
\Psi: M^{\ell} \rightarrow Z_{0}(M)
$$

is the subgroup generated by the various $m_{\alpha^{\ell}}$ with $\alpha$ long. If $\beta$ is short, then

$$
\Psi\left(m_{\beta^{\ell}}\right)=m_{\beta}
$$


Consequently the transpose map

$$
\Psi^{t}:\left\{\text { characters of } Z_{0}(M)\right\} \hookrightarrow\left\{\text { characters of } M^{\ell}\right\}
$$

has as image precisely the characters of $M^{\ell}$ trivial on $m_{\alpha^{\ell}}$ with $\alpha$ long. In particular,

$\Psi^{t}:\left\{\right.$ characters of $Z_{0}(M)$ trivial on $m_{\beta}$ for $\beta$ short $\} \hookrightarrow\left\{\right.$ characters of $\left.M^{\ell}\right\}$

has as image precisely the pseudospherical characters of $M^{\ell}$.

(3) For any $G$, define $\bar{Z}_{0}(M)$ to be the quotient of $Z_{0}(M)$ by the subgroup generated by the elements $m_{\beta}$ with $\beta$ not metaplectic (Lemma 4.11). Then the transpose map

$$
\Psi^{t}:\left\{\text { characters of } \bar{Z}_{0}(M)\right\} \hookrightarrow\left\{\text { characters of } M^{\ell}\right\}
$$

has as image precisely the pseudospherical characters of $M^{\ell}$.

(4) There is a natural simply transitive action of the group of pseudospherical characters of $M^{\ell}$ on the set of genuine pseudospherical representations of $\widetilde{M}$, as follows. Given a pseudospherical character $\delta^{\ell}$ of $M^{\ell}$, choose a character $\delta$ of $M$ so that $\Psi^{t}(\delta)=\delta^{\ell}$. Regard $\delta$ as a (non-genuine) character of $\widetilde{M}$. Then if $\tilde{\delta}$ is any genuine pseudospherical representation of $\widetilde{M}$, we define

$$
\delta^{\ell} \cdot \tilde{\delta}=\delta \otimes \tilde{\delta}
$$

Proof. Parts (1) and (2) are a straightforward consequence of the calculation of $\Gamma$ in Lemma 3.5 and of Definition 6.1 we leave the details to the reader. Parts (3) and (4) then follow immediately from Lemma 4.11.

In Table 1, the column $\left|\Pi_{g}(\widetilde{M})\right|$ counts irreducible genuine representations of $\widetilde{M} ;\left|\Pi_{g s}(\widetilde{M})\right|$ counts irreducible genuine pseudospherical representations of $\widetilde{M}$; and

Dim is the dimension of an irreducible genuine representation of $\widetilde{M}$. In Table 3 , the column $\left|\Pi_{s}\left(M^{\ell}\right)\right|$ counts the irreducible pseudospherical representations of $M^{\ell}$.

TABLE 1. Genuine representations of $\widetilde{M}$

\begin{tabular}{lllll} 
Type & $G$ & $\left|\Pi_{g}(\widetilde{M})\right|$ & $\left|\Pi_{g s}(\widetilde{M})\right|$ & $\operatorname{Dim}$ \\
\hline$A_{2 m-1}$ & $\operatorname{SL}(2 m)$ & 2 & 2 & $2^{m-1}$ \\
$A_{2 m}$ & $\operatorname{SL}(2 m+1)$ & 1 & 1 & $2^{m}$ \\
$B_{2 m}$ & $\operatorname{Spin}(2 m+1,2 m)$ & 4 & 2 & $2^{m-1}$ \\
$B_{2 m-1}$ & $\operatorname{Spin}(2 m, 2 m-1)$ & 2 & 1 & $2^{m-1}$ \\
$C_{n}$ & $\operatorname{Sp}(2 n)$ & $2^{n}$ & 2 & 1 \\
$D_{2 m}$ & $\operatorname{Spin}(2 m, 2 m)$ & 4 & 4 & $2^{m-1}$ \\
$D_{2 m+1}$ & $\operatorname{Spin}(2 m+1,2 m+1)$ & 2 & 2 & $2^{m}$ \\
$E_{6}$ & $E_{6}\left(C_{4}\right)$ & 1 & 1 & 8 \\
$E_{7}$ & $E_{7}\left(A_{7}\right)$ & 2 & 2 & 8 \\
$E_{8}$ & $E_{7}\left(D_{8}\right)$ & 1 & 1 & 16 \\
$F_{4}$ & $F_{4}\left(A_{1} \times C_{3}\right)$ & 4 & 1 & 2 \\
$G_{2}$ & $G_{2}\left(A_{1} \times A_{1}\right)$ & 1 & 1 & 2 \\
\hline
\end{tabular}


TABLE 2. Pseudospherical representations of $\widetilde{K}$

\begin{tabular}{llll}
$G$ & $\widetilde{K}$ & $\tilde{\mu}$ & $\operatorname{Dim}(\tilde{\mu})$ \\
\hline $\operatorname{SL}(2 m)$ & $\operatorname{Spin}(2 m)$ & $\operatorname{Spin}_{ \pm}$ & $2^{m-1}$ \\
$\operatorname{SL}(2 m+1)$ & $\operatorname{Spin}(2 m+1)$ & $\operatorname{Spin}^{m}$ \\
$\operatorname{Spin}(2 m+1,2 m)$ & $\operatorname{Spin}(2 m+1) \times \operatorname{Spin}(2 m)$ & $1 \otimes \operatorname{Spin}_{ \pm}$ & $2^{m-1}$ \\
$\operatorname{Spin}(2 m, 2 m-1)$ & $\operatorname{Spin}(2 m) \times \operatorname{Spin}(2 m-1)$ & $1 \otimes \operatorname{Spin}^{m-1}$ & $2^{m}$ \\
$\operatorname{Sp}(2 n)$ & $\mathrm{U}(n)$ & $\operatorname{det}^{ \pm \frac{1}{2}}$ & 1 \\
$\operatorname{Spin}(2 m, 2 m)$ & $\operatorname{Spin}(2 m) \times \operatorname{Spin}(2 m)$ & $1 \otimes \operatorname{Spin}_{ \pm}$, & \\
& & $\operatorname{Spin}_{ \pm} \otimes 1$ & $2^{m-1}$ \\
$\operatorname{Spin}(2 m+1,2 m+1)$ & $\operatorname{Spin}(2 m+1) \times \operatorname{Spin}(2 m+1)$ & $1 \otimes \operatorname{Spin}$, & \\
& & $\operatorname{Sppin} \otimes 1$ & $2^{m}$ \\
$E_{6}$ & $\operatorname{Sp}(8)$ & $\mathbb{C}^{8}$ & 8 \\
$E_{7}$ & $\operatorname{SU}(8)$ & $\mathbb{C}^{8},\left(\mathbb{C}^{8}\right)^{*}$ & 8 \\
$E_{8}$ & $\operatorname{Spin}(16)$ & $\mathbb{C}^{16}$ & 16 \\
$F_{4}$ & $\operatorname{Sp}(6) \times \operatorname{Spin}(3)$ & $1 \otimes \operatorname{Spin}$ & 2 \\
$G_{2}$ & $\operatorname{Spin}(3) \times \operatorname{Spin}(3)$ & $\operatorname{Spin} \otimes 1$ & 2 \\
\hline
\end{tabular}

TABLE 3 . The linear reincarnation $G^{\ell}$

\begin{tabular}{llll}
$G$ & $G^{\ell}$ & $\left|\Pi_{s}\left(M^{\ell}\right)\right|$ & \\
\hline $\operatorname{SL}(n)$ & $\operatorname{PGL}(n)$ & 2 & $n$ even \\
& $\operatorname{SL}(n)$ & 1 & $n$ odd \\
$\operatorname{Spin}(n+1, n)$ & $\operatorname{PSp}(2 n)$ & 2 & $n$ even \\
& $\operatorname{Sp}(2 n)$ & 1 & $n$ odd \\
$\operatorname{Sp}(2 n)$ & $\operatorname{SO}(n+1, n)$ & 2 & \\
$\operatorname{Spin}(n, n)$ & $\operatorname{PSO}(n, n)$ & 4 & $n$ even \\
& $\operatorname{SO}(n, n)$ & 2 & $n$ odd \\
$E_{6}^{s c}$ & $E_{6}^{s c}$ & 1 & \\
$E_{7}^{s c}$ & $E_{7}^{a d}$ & 2 & \\
$E_{8}$ & $E_{8}$ & 1 & \\
$F_{4}$ & $F_{4}$ & 1 & \\
$G_{2}$ & $G_{2}$ & 1 & \\
\hline
\end{tabular}

\section{A CONJECTURAL Shimura CORRESPONDENCE}

Retain the notation of Definition 1.1 and Section 4 Fix an irreducible genuine pseudospherical representation $\tilde{\delta}$ of $\widetilde{M}$ and let $\mu(\tilde{\delta})$ denote its pseudospherical extension to $\widetilde{K}$ (Proposition [5.2). Recall from Definition [5.5 that $J(\tilde{\delta}, \nu)$ is defined to be the composition factor of $I(\tilde{\delta}, \nu)$ containing $\mu(\tilde{\delta})$. Let

$$
\mathbf{C S}(\tilde{\delta}, \widetilde{G})=\left\{\nu \in \mathfrak{a}_{\mathbb{C}}^{*} \mid J(\tilde{\delta}, \nu) \text { is unitary }\right\},
$$

the pseudospherical complementary series of $\widetilde{G}$. Lemma $4.11(3)$ implies that

$$
J(\tilde{\delta}, \nu) \simeq J\left(\tilde{\delta}, \nu^{\prime}\right)
$$

if and only if there exists $w \in W \operatorname{such}$ that $\nu^{\prime}=w \cdot \nu$. Consequently $\operatorname{CS}(\widetilde{G}, \tilde{\delta})$ is a closed $W$-invariant set. 
Conjecture 7.1. Let $G^{\ell}$ be given as in Section 6. Let $\Psi: A^{\ell} \rightarrow A$ be given by Lemma 6.3, with adjoint $d \Psi^{t}: \mathfrak{a}_{\mathbb{C}}^{*} \rightarrow\left(\mathfrak{a}^{\ell}\right)_{\mathbb{C}}^{*}$. Fix a pseudospherical representation $\delta^{\ell}$ of $M^{\ell}$. Then $d \Psi^{t}$ defines a bijection:

$$
\mathbf{C S}(\tilde{\delta}, \widetilde{G}) \rightarrow \mathbf{C S}\left(\delta^{\ell}, G^{\ell}\right) .
$$

That is, $J_{\widetilde{G}}(\tilde{\delta}, \nu)$ is unitary if and only if $J_{G^{\ell}}\left(\delta^{\ell}, d \Psi^{t}(\nu)\right)$ is unitary.

By Lemma 4.20, $\mathbf{C S}\left(\delta^{\ell}, G^{\ell}\right)$ is independent of $\delta^{\ell}$ and is equal to the spherical complementary series of the identity component $\mathbf{C S}\left(\left(G^{\prime}\right)^{0}\right)$ of any linear group $G^{\prime}$ locally isomorphic to $G^{\ell}$.

If $G$ is simply laced or $G_{2}$, then Conjecture 7.1 is equivalent to: $J_{\widetilde{G}}(\tilde{\delta}, \nu)$ is unitary if and only if the spherical representation $J_{G}(2 \nu)$ is unitary, i.e.,

$$
2 \mathbf{C S}(\tilde{\delta}, \widetilde{G})=\mathbf{C S}(G) .
$$

Example 7.2. Let $G=\operatorname{Sp}(2 n, \mathbb{R})$ and $G^{\ell}=\mathrm{SO}(n+1, n)$. As discussed in the introduction, $\Psi: A^{\ell} \simeq A$ is an isomorphism in this case, given by the identity map in the usual coordinates. See Section 12.2 .

Note that $\rho$ (one-half the sum of the positive roots) for $\mathrm{SO}(n+1, n)$, i.e., $\left(n-\frac{1}{2}, \ldots, \frac{3}{2}, \frac{1}{2}\right)$, is the image under $d \Psi^{t}$ of the Harish-Chandra parameter of the infinitesimal character of the oscillator representation of $\widetilde{\operatorname{Sp}}(2 n, \mathbb{R})$. The trivial representation, which is an isolated point in the unitary dual of $\mathrm{SO}(n+1, n)($ for $n \geq 2)$ corresponds to one of the components of the oscillator representation.

For example if $n=2$, the complementary series $\mathbf{C S}(\tilde{\delta}, \widetilde{\operatorname{Sp}}(4, \mathbb{R}))=\mathbf{C S}(\operatorname{SO}(3,2))$ looks like the diagram in Figure 1.

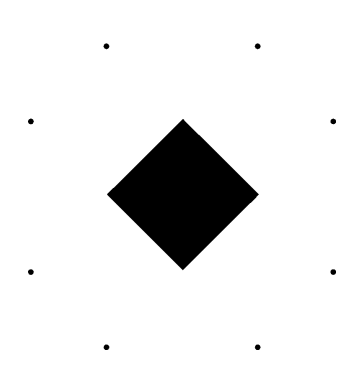

Figure 1. Spherical complementary series for $\mathrm{SO}(3,2)$

If $G$ is a classical group we will prove the inclusion

$$
d \Psi^{t}(\mathbf{C S}(\tilde{\delta}, \widetilde{G})) \subset \mathbf{C S}\left(\delta^{\ell}, G^{\vee}\right)
$$

of Conjecture 7.1 (Theorem [13.24), and equality for $\widetilde{G}=\widetilde{\mathrm{Sp}}(2 n)$ (Theorem 14.2).

Remark 7.3. We conclude this section with a discussion of the $p$-adic analog of Conjecture 7.1. Let $F$ be a $p$-adic field with ring of integers $R$, and let $G$ be a split simple algebraic group defined over $R$. Fix an integer $n$ relatively prime to $p$ such that $\mu_{n}(F)$, the $n$th roots of unity in $F$, has order $n$. It follows from [Ma that there is a "metaplectic" extension $\widetilde{G}$ of $G$ by $\mu_{n}(F)$ (see, for example, [Sa, 2.1]). Let $I$ denote an Iwahori subgroup of $G$, and $\widetilde{I}$ its preimage in $\widetilde{G}$. The extension splits canonically over $I$, and we use this splitting to regard $I$ as a subgroup of $\widetilde{G}$. The 
category of smooth representations of $G$ generated by their $I$-fixed vectors is equivalent to the category of representation of the Hecke algebra $\mathcal{H}(G)$ of $I$ bi-invariant compactly supported functions on $G$. Likewise, the category of smooth (suitably genuine) representations of $\widetilde{G}$ generated by their $I$-fixed vectors is equivalent to the category of representations of $\mathcal{H}(\widetilde{G})$, the (suitably genuine) $I$-invariant compactly supported functions on $\widetilde{G}$. Savin has predicted and investigated the existence of an isomorphism between $\mathcal{H}\left(G^{\ell}\right)$ and $\mathcal{H}(\widetilde{G})$. This is very subtle; the arguments offered in $\mathrm{Sa}$ may not be complete. When $G$ is simply laced, the situation is simpler and Savin has proved in $\left[\right.$ Sa2, Theorem 7.8] that $\mathcal{H}\left(G^{\ell}\right) \simeq \mathcal{H}(\widetilde{G})$. The conclusion in these cases is that there is an equivalence between the category of (suitably genuine) smooth representations of $\widetilde{G}$ generated by their $I$-fixed vectors and the category of smooth representations of $G^{\ell}$ generated by their $I^{\ell}$-fixed vectors. The $p$-adic analog of Conjecture 7.1 is that this equivalence should preserve unitarity (cf. [Hu2]).

\section{REDUCTION TO REAL INFINITESIMAL CHARACTER}

We continue in the setting of Conjecture 7.1. Recall from [ $\mathrm{Kn}$, Section 14.4] the notion of representations with real infinitesimal character. In our setting this is extremely straightforward: $J(\tilde{\delta}, \nu)$ has real infinitesimal character if and only if $\nu \in \mathfrak{a}^{*} \subset \mathfrak{a}_{\mathbb{C}}^{*}$, equivalently, if $\left\langle\nu, \alpha^{\vee}\right\rangle \in \mathbb{R}$ for all roots $\alpha$. In this case we say $\nu$ is real. Similarly, we say $\nu$ is purely imaginary if $\nu \in i \mathfrak{a}^{*}$, that is, if $i \nu$ is real. Many questions about the classification of unitary representations can be reduced to the case of real infinitesimal character. Our purpose in this section is to do that for Conjecture 7.1. Here is the statement.

Proposition 8.1. Suppose $G$ is a simply connected split real group as in Definition 1.1. For any connected subset $E$ of the Dynkin diagram of $G$, let $G_{E}$ be the subgroup of $G$ corresponding to the roots spanned by $E$; this is a simply connected split real group, with split torus $A_{E} \subset A$. Assume that Conjecture 7.1 holds for each group $G_{E}$ and real $\nu_{E} \in \mathfrak{a}^{*}$. Then Conjecture 7.1 holds for $G$.

Proof. That each $G_{E}$ is split is clear. That $G_{E}$ is simply connected follows from the fact that each fundamental weight for $G_{E}$ (corresponding to a vertex of its Dynkin diagram) is the restriction to $A_{E}$ of the corresponding fundamental weight for $G$. We write $\left(G^{\ell}\right)_{E}$ for the corresponding algebraic subgroup of $G^{\ell}$, and $\widetilde{G}_{E}$ for the preimage of $G_{E}$ in $\widetilde{G}$. The group $\widetilde{G}_{E}$ is a two-fold cover of $G_{E}$ as in Definition 4.1, so it is either trivial or equal to the distinguished nontrivial cover of Definition 1.1. Similarly, the group $\left(G^{\ell}\right)_{E}$ has the same Weyl group as $G_{E}$, but it may or may not be the group $\left(G_{E}\right)^{\ell}$ constructed in Definition 6.1. We will say more about these questions at the end of the proof.

We first describe how to relate the unitarity of pseudospherical representations of $\widetilde{G}$ to the unitarity of pseudospherical representations of the groups $\widetilde{G}_{E}$ with real infinitesimal character.

Fix $\nu \in \mathfrak{a}_{\mathbb{C}}^{*}$ and write $\nu=\nu_{\mathbb{R}}+i \nu_{I}$ with $\nu_{\mathbb{R}}, \nu_{I}$ real. After conjugating $\nu$ by $W$, we may assume that $\nu_{I}$ is dominant. Let $L$ be the Levi subgroup of $G$ corresponding to the roots $\left\{\alpha \mid\left\langle\nu_{I}^{\ell}, \alpha^{\vee}\right\rangle=0\right\}$, and let $P$ be the corresponding standard parabolic subgroup. Then the spherical representations $I_{\widetilde{L}}(\tilde{\delta}, \nu)$ and $J_{\widetilde{L}}(\tilde{\delta}, \nu)$ are defined, and

$$
I_{\widetilde{G}}(\tilde{\delta}, \nu)=\operatorname{Ind}_{\widetilde{P}}^{\widetilde{G}}\left(I_{\widetilde{L}}(\tilde{\delta}, \nu)\right) .
$$


Furthermore

$$
J_{\widetilde{G}}(\tilde{\delta}, \nu)=\operatorname{Ind}_{\widetilde{P}}^{\widetilde{G}}\left(J_{\widetilde{L}}(\tilde{\delta}, \nu)\right)
$$

and

$$
J_{\widetilde{G}}(\tilde{\delta}, \nu) \text { is unitary if and only if } J_{\widetilde{L}}(\tilde{\delta}, \nu) \text { is unitary. }
$$

This is contained in [Kn, Theorem 16.10]; the assumption there that the group is linear is not important.

Now let $E$ be the subset of the Dynkin diagram corresponding to $L$ (consisting of the simple roots vanishing on $\nu_{I}$ ). Write this set as the disjoint union of its connected components:

$$
E=E_{1} \cup \cdots \cup E_{r} .
$$

Let $Z \subset A$ be the center of $L$. Then the identity component of $\widetilde{L}$ is finitely covered by the identity component of the product group $Z \times \prod_{i=1}^{r} \widetilde{G}_{E_{i}}$. We have

$$
\mathfrak{a} \simeq \mathfrak{z} \oplus \sum_{i=1}^{r} \mathfrak{a}_{E_{i}}, \quad \nu=\nu_{Z}+\sum_{i=1}^{r} \nu_{i},
$$

with $\nu_{Z} \in \mathfrak{z}_{\mathbb{C}}^{*}$ and $\nu_{i} \in\left(\mathfrak{a}_{E_{i}}\right)_{\mathbb{C}}^{*}$. Fix some irreducible constituent $\tilde{\delta}_{i}$ of the restriction of $\tilde{\delta}$ to $\widetilde{M} \cap \widetilde{G}_{E_{i}}$. Standard facts about unitary representations of product groups show that

$$
J_{\widetilde{L}}(\tilde{\delta}, \nu) \text { unitary } \Leftrightarrow \nu_{Z} \text { imaginary and each } J_{\widetilde{G}_{E_{i}}}\left(\tilde{\delta}_{i}, \nu_{i}\right) \text { unitary. }
$$

The construction of $L$ shows also that each $\nu_{i}$ is real.

Equations (8.2) provide a reduction of the classification of unitary pseudospherical representations of $\widetilde{G}$ to parallel problems for simple Levi subgroups and real infinitesimal characters. We turn next to a similar reduction for $G^{\ell}$.

As in Conjecture [7.1] we fix a pseudospherical character $\delta^{\ell}$ of $M^{\ell}$, and define $\nu^{\ell}=d \Psi^{t}(\nu) \in\left(\mathfrak{a}^{\ell}\right)_{\mathbb{C}}^{*}$. Note that $d \Psi^{t}\left(\nu_{R}\right)=\nu_{R}^{\ell}$ and $d \Psi^{t}\left(\nu_{I}\right)=\nu_{I}^{\ell}$. The fundamental fact is that $d \Psi$ carries each coroot $\left(\alpha^{\ell}\right)^{\vee}$ for $G^{\ell}$ to a positive multiple of the corresponding coroot $\alpha^{\vee}$ for $G$. This means first of all that $\nu_{I}^{\ell}$ is dominant and that the Levi subgroup $L^{\ell}$ defined by $\nu_{I}^{\ell}$ corresponds to the subset $\left\{\alpha^{\ell} \mid \alpha \in E\right\}$ of the Dynkin diagram for $G^{\ell}$. The pseudospherical representations $I_{L^{\ell}}\left(\delta^{\ell}, \nu^{\ell}\right)$ and $J_{L^{\ell}}\left(\delta^{\ell}, \nu^{\ell}\right)$ are defined, and

$$
I_{G^{\ell}}\left(\delta^{\ell}, \nu^{\ell}\right)=\operatorname{Ind}_{P^{\ell}}^{G^{\ell}}\left(I_{L^{\ell}}\left(\delta^{\ell}, \nu^{\ell}\right)\right), \quad J_{G^{\ell}}\left(\delta^{\ell}, \nu^{\ell}\right)=\operatorname{Ind}_{P^{\ell}}^{G^{\ell}}\left(J_{L^{\ell}}\left(\delta^{\ell}, \nu^{\ell}\right)\right) .
$$

We have

$$
J_{G^{\ell}}\left(\nu^{\ell}\right) \text { is unitary if and only if } J_{L^{\ell}}\left(\nu^{\ell}\right) \text { is unitary. }
$$

Again this is found in $[\mathrm{Kn}$, Theorem 16.10]; this time what is unimportant is the assumption there that $G$ is connected.

Because the Dynkin diagrams of $G$ and $G^{\ell}$ are the same except for the orientation of arrows, the connected components of $E$ in the Dynkin diagram of $G^{\ell}$ are precisely the various $E_{i}$. Let $Z^{\ell}$ be the center of $L^{\ell}$. Then $L^{\ell}$ is finitely covered by $Z^{\ell} \times$ $\prod_{i=1}^{r}\left(G^{\ell}\right)_{E_{i}}$, and we have

$$
\mathfrak{a}^{\ell} \simeq \mathfrak{z}^{\ell} \oplus \sum_{i=1}^{r} \mathfrak{a}_{E_{i}}^{\ell} .
$$

The map $\Psi: A^{\ell} \rightarrow A$ of Definition 6.1 restricts to

$$
\Psi_{Z}: Z^{\ell} \rightarrow Z, \quad \Psi_{i}: A_{E_{i}}^{\ell} \rightarrow A_{E_{i}} .
$$


Accordingly we can write

$$
\nu^{\ell}=\nu_{Z}^{\ell}+\sum_{i=1}^{r} \nu_{i}^{\ell}, \quad \nu_{Z}^{\ell}=d \Psi_{Z}^{t}\left(\nu_{Z}\right) \in \mathfrak{z}_{\mathbb{C}}^{*}, \quad \nu_{i}^{\ell}=d \Psi_{i}^{t}\left(\nu_{i}\right) \in\left(\mathfrak{a}_{E_{i}}^{\ell}\right)_{\mathbb{C}}^{*}
$$

Just as in 8.2$)(\mathrm{b})$,

(8.3)(b) $J_{L^{\ell}}\left(\delta^{\ell}, \nu^{\ell}\right)$ unitary $\Leftrightarrow \nu_{Z}^{\ell}$ imaginary and each $J_{\left(G^{\ell}\right)_{E_{i}}}\left(\delta_{i}^{\ell}, \nu_{i}^{\ell}\right)$ unitary;

here $\delta_{i}^{\ell}$ is the restriction of the character $\delta^{\ell}$ to $M^{\ell} \cap\left(G^{\ell}\right)_{E_{i}}$.

Equations (8.3) reduce the classification of unitary pseudospherical representations of $G^{\ell}$ to parallel problems for simple Levi subgroups and real infinitesimal characters.

In order to complete the proof of Proposition 8.1, we must show that the unitarity criteria in (8.2) and (8.3) agree. Because $d \Psi$ preserves real and imaginary parts, we have immediately that

$$
\nu_{Z} \text { is imaginary } \Leftrightarrow \nu_{Z}^{\ell} \text { is imaginary. }
$$

Next, fix one of the $E_{i}$. There are two possibilities. If no root in $E_{i}$ is metaplectic, then the covering $\widetilde{G}_{E_{i}}$ is trivial, so $J_{(\widetilde{G})_{E_{i}}}\left(\tilde{\delta}_{i}, \nu_{i}\right)$ is unitary if and only if the spherical representation $J_{G_{E_{i}}}\left(\nu_{i}\right)$ is unitary. According to (6.2), the map $\Psi_{i}$ carries the coroots of $G_{E_{i}}^{\ell}$ onto those of $G_{E_{i}}$. Consequently $G_{E_{i}}$ finitely covers the identity component of $G_{E_{i}}^{\ell}$, and this covering identifies $\nu_{i}$ with $\nu_{i}^{\ell}$. It follows immediately that

$$
J_{\widetilde{G}_{E_{i}}}\left(\tilde{\delta}_{i}, \nu_{i}\right) \text { is unitary } \Leftrightarrow J_{\left(G^{\ell}\right)_{E_{i}}}\left(\delta_{i}^{\ell}, \nu_{i}^{\ell}\right) \text { is unitary. }
$$

Finally, assume that some root of $E_{i}$ is metaplectic. It follows that the covering $\widetilde{G}_{E_{i}}$ is nontrivial, so it must be the covering of Definition 1.1. The map $\Psi_{i}$ inherits the critical properties (6.2) from $\Psi$, and it follows easily that $\left(G^{\ell}\right)_{E_{i}}$ is locally isomorphic to the linear group $\left(G_{E_{i}}\right)^{\ell}$ constructed in Definition 6.1 The assertion,

$$
J_{\widetilde{G}_{E_{i}}}\left(\tilde{\delta}_{i}, \nu_{i}\right) \text { is unitary } \Leftrightarrow J_{\left(G^{\ell}\right)_{E_{i}}}\left(\delta_{i}^{\ell}, \nu_{i}^{\ell}\right) \text { is unitary, }
$$

in this case is equivalent to Conjecture 7.1 for the group $G_{E_{i}}$ and the real parameter $\nu_{i}$. The hypothesis of the proposition is that this statement holds.

Equations (8.4) show that the unitarity criteria for $\nu$ in $\widetilde{G}$ and $\nu^{\ell}$ in $G^{\ell}$ match perfectly. The proposition follows.

\section{Petite Representations of the Weyl group}

Suppose $R$ is a root system, with Weyl group $W$. Suppose $\alpha, \beta$ are adjacent roots. The subgroup $\left\langle s_{\alpha}, s_{\beta}\right\rangle$ of $W$ generated by $s_{\alpha}, s_{\beta}$ is isomorphic to the Weyl group of type $A_{2}, B_{2}$ or $G_{2}$, and so has a one-dimensional sign representation.

Definition 9.1. Let $\sigma$ be a representation of $W$. We say $\sigma$ is petite if

(1) for all pairs $\alpha, \beta$ of adjacent simple roots, the restriction of $\sigma$ to $\left\langle s_{\alpha}, s_{\beta}\right\rangle$ does not contain the sgn representation; and

(2) if $\alpha$ and $\beta$ are adjacent long roots inside a subsystem of type $G_{2}$, then the restriction of $\sigma$ to $\left\langle s_{\alpha}, s_{\beta}\right\rangle$ does not contain the sgn representation. 
Condition (2) excludes the one-dimensional character of $W\left(G_{2}\right)$ acting on the product of a set of long positive roots. The one-dimensional character of $W\left(G_{2}\right)$ on the product of a set of short positive roots is allowed; it would have been excluded if we omitted the word "simple" in (1). For root systems not containing factors of type $G_{2}$, the word "simple" in (1) has no effect since any pair of adjacent roots is (up to sign) conjugate by $W$ to a pair of simple roots.

The motivation for this terminology will be given in Section10 (cf. Lemma 10.9).

9.1. Petite Representations of Classical Weyl Groups. We recall some notation from [Ba1]. The classical Weyl groups are all realized as linear transformations of $\mathfrak{a} \simeq \mathbb{R}^{n}$. The hyperoctahedral group $W\left(B_{n}\right)=W\left(C_{n}\right)$ includes all $2^{n} \cdot n$ ! such transformations. The symmetric group $W\left(A_{n-1}\right) \simeq S_{n}$ changes no signs, and $W\left(D_{n}\right)$ is the subgroup of $W\left(B_{n}\right)$ changing an even number of signs.

For $(a, b)$ a partition of $n$ we let $(a, b)$ denote the corresponding irreducible representation of $W\left(A_{n-1}\right) \simeq S_{n}$, of dimension $\left(\begin{array}{l}n \\ a\end{array}\right)(n-2 a+1) /(n-a+1)$. (Representations attached to partititions into more than two parts contain the sign character of $S_{3}$, and so are not petite.) The representation $(n)$ is the trivial representation of $S_{n}$, and $(n-1,1)$ is the $(n-1)$-dimensional reflection representation. We use the same notation to denote $(a, b)$ pulled back to $W\left(B_{n}\right)=W\left(C_{n}\right)$ or $W\left(D_{n}\right)$ via the natural surjections to $S_{n}$.

Let $\chi_{n}$ denote the character of $W\left(B_{n}\right)$ satisfying $\chi\left(s_{\alpha}\right)=1$ (resp. -1 ) if $\alpha$ is long (resp. short). The Weyl group $W\left(D_{n}\right)$ is the kernel of $\chi_{n}$.

Fix nonnegative integers $a, b$ satisfying $a+b=n$. We embed $W\left(B_{a}\right) \times W\left(B_{b}\right)$ in $W\left(B_{n}\right)$ as usual. Let $(a) \times(b)=\operatorname{Ind}_{W\left(B_{a}\right) \times W\left(B_{b}\right)}^{W\left(B_{n}\right)}\left(\mathbf{1} \otimes \chi_{b}\right)$. These representations are irreducible and distinct, and $(a) \times(b) \simeq \chi_{n} \otimes((b) \times(a))$. The dimension of $(a) \times(b)$ is $\left(\begin{array}{l}n \\ a\end{array}\right)$.

We also write $(a) \times(b)$ for the restriction of $(a) \times(b)$ to $W\left(D_{n}\right)$. As representations of $W\left(D_{n}\right),(a) \times(b) \simeq(b) \times(a)$, and this representation is irreducible unless $a=b$. If $n$ is even, the representation $(n / 2) \times(n / 2)$ of $W\left(B_{n}\right)$ restricts to the direct sum of two irreducible representations $(n / 2) \times(n / 2)_{ \pm}$of $W\left(D_{n}\right)$, each of dimension $\left(\begin{array}{c}n \\ n / 2\end{array}\right) / 2$.

Proposition 9.2. Every irreducible petite representation of a classical Weyl group is isomorphic to $(a, b),(a) \times(b)$ or $(n / 2) \times(n / 2)_{ \pm}$.

We omit a proof of this proposition. Our only application of petite Weyl group representations is in the proof of Theorem 13.24 (cf. Proposition 12.11). For these purposes it is enough to use the condition of Proposition 9.2 as the definition of petite.

\section{Action of the Weyl group}

We work in the setting of Definition 4.1 so $\widetilde{G}$ is a linear or nonlinear group. Fix a genuine pseudospherical representation $\left(\tilde{\delta}, F_{\tilde{\delta}}\right)$ of $\widetilde{M}$ and let $\left(\mu_{0}, F_{\tilde{\delta}}\right)$ be its unique pseudospherical extension to $\widetilde{K}$ (Lemma 4.19 and Proposition [5.2).

Let $\widetilde{M}^{\prime}$ be the normalizer of $\widetilde{A}$ in $\widetilde{K}$. We have an exact sequence

$$
1 \rightarrow \widetilde{M} \rightarrow \widetilde{M^{\prime}} \rightarrow W \rightarrow 1 .
$$

The following proposition is a basic result in Clifford theory; for example, see CR, Theorem 51.7]. 
Lemma 10.2. There is a bijection between the irreducible representations of $W$ and the irreducible representations of $\widetilde{M}^{\prime}$ whose restriction to $\widetilde{M}$ contains $\tilde{\delta}$. The bijection may be constructed as follows.

If $\left(\sigma, E_{\sigma}\right)$ is an irreducible representation of $W$, let $E_{\tau}=E_{\sigma} \otimes F_{\tilde{\delta}}$, and define

$$
\tau(g)(v \otimes w)=\sigma(g)(v) \otimes \mu_{0}(g)(w) \quad\left(v \in F_{\tilde{\delta}}, w \in E_{\sigma}, g \in \widetilde{M}^{\prime}\right) .
$$

Conversely, suppose $\left(\tau, E_{\tau}\right)$ is an irreducible representation of $\widetilde{M}^{\prime}$ containing $\tilde{\delta}$. Define a representation $\sigma$ of $\widetilde{M}^{\prime}$ on $\operatorname{Hom}_{\widetilde{M}}\left(F_{\tilde{\delta}}, E_{\tau}\right)$ by

$$
\sigma(n)(\phi)=\tau(n) \circ \phi \circ \mu_{0}\left(n^{-1}\right) \quad\left(n \in \widetilde{M}^{\prime}, \phi \in \operatorname{Hom}_{\widetilde{M}}\left(F_{\tilde{\delta}}, E_{\tau}\right)\right) .
$$

Then $\sigma$ factors to a representation of $W$, and

$$
\operatorname{Hom}_{\widetilde{M}}\left(F_{\tilde{\delta}}, E_{\tau}\right) \otimes F_{\tilde{\delta}} \simeq E_{\tau}
$$

as representations of $\widetilde{M}^{\prime}$, via the map $\phi \otimes v \rightarrow \phi(v)$.

Remark 10.3. If we begin only with the exact sequence (10.1) and an irreducible representation $\tilde{\delta}$ of $\widetilde{M}$, then the bijection of the lemma depends on the existence and choice of a representation of $\widetilde{M}^{\prime}$ extending $\tilde{\delta}$. In our setting we can use the canonically defined extension $\mu_{0}$ of $\tilde{\delta}$ to $\widetilde{K}$. This is what makes the bijection of the theorem canonical.

Now consider the principal series representation $I(\tilde{\delta}, \nu)$. The $\mu$-isotypic component of $I(\tilde{\delta}, \nu)$ is given by

$$
\operatorname{Hom}_{\widetilde{K}}\left(V_{\mu}, I(\tilde{\delta}, \nu)\right) \otimes V_{\mu},
$$

which by Frobenius reciprocity is isomorphic to

$$
\operatorname{Hom}_{\widetilde{M}}\left(V_{\mu}, F_{\tilde{\delta}}\right) \otimes V_{\mu} .
$$

Definition 10.5. Suppose $\mu$ is a representation of $\widetilde{K}$. Write

$$
V_{\mu}[\tilde{\delta}]=\operatorname{Hom}_{\widetilde{M}}\left(V_{\mu}, F_{\tilde{\delta}}\right) \simeq \operatorname{Hom}_{\widetilde{K}}\left(V_{\mu}, I(\tilde{\delta}, \nu)\right) .
$$

The Weyl group representation $\sigma(\mu)$ attached to $\mu$ and $\tilde{\delta}$ is defined on this space as in Lemma 10.2 .

$$
\sigma(n)(\phi)=\mu_{0}(n) \circ \phi \circ \mu\left(n^{-1}\right) \quad\left(n \in \widetilde{M}^{\prime}, \phi \in V_{\mu}[\tilde{\delta}]\right) .
$$

The representation $\sigma$ is trivial on $\widetilde{M}$ and therefore factors to a representation of $W$ on $V_{\mu}[\tilde{\delta}]$.

The Weyl group representation $\sigma(\mu)$ need not be irreducible. According to Lemma 10.2. it is irreducible if and only if the representation of $\widetilde{M}^{\prime}$ on the $\tilde{\delta}$ isotypic subspace of $\mu$ is irreducible.

We are interested in the representations of $W$ attached to petite representations of $\widetilde{K}$.

Definition 10.7. Let $\Sigma_{\widetilde{K}}(\widetilde{G}, \tilde{\delta})$ be the set of irreducible petite representations of $\widetilde{K}$ containing the pseudospherical $\widetilde{M}$ representation $\tilde{\delta}$. Let

$$
\Sigma_{W}(\widetilde{G}, \tilde{\delta})=\left\{\sigma \in \widehat{W} \mid \sigma \subset V_{\mu}[\tilde{\delta}] \text { for some } \mu \in \Sigma_{\widetilde{K}}(\widetilde{G}, \tilde{\delta})\right\}
$$


Lemma 10.9 ([Ba1]). Suppose $G$ is a linear classical group, and $\delta$ a pseudospherical character of $M$. Then $\Sigma_{W}(G, \delta)$ is the set of petite representations of $W$.

(In fact, if $\mu$ is an irreducible petite representation of $K$ containing the pseudospherical $M$ representation $\delta$, then $V_{\mu}[\delta]$ is an irreducible petite representation of $W$.

Remark 10.10. The same result holds for exceptional groups (with a slight modification in type $G_{2}$ ), but the calculations necessary to prove this are too long to include here.

We discuss nonlinear groups in the next two sections.

\section{Construction of Petite $\widetilde{K}$-types: Simply laced CASE}

We continue with the setting of the previous section, where now $\widetilde{G}$ is a nonlinear group. We find a relationship between genuine petite representations of $\widetilde{K}$, pseudospherical representations of $\widetilde{M}$, and petite representations of $W$.

Proposition 11.1. Assume $G$ is simply laced, and that $\widetilde{G}$ is the nonlinear cover of Definition 1.1. Let $S=\{(\tilde{\delta}, \sigma)\}$ where $\tilde{\delta}$ is a genuine pseudospherical representation of $\widetilde{M}$ and $\sigma$ is a petite representation of $W$. There is a bijection between $S$ and the genuine irreducible petite representations of $\widetilde{K}$.

Explicitly, suppose $\mu$ is such a representation of $\widetilde{K}$. Necessarily the restriction of $\mu$ to $\widetilde{M}$ is a multiple of a genuine pseudospherical representation $\tilde{\delta}$ of $\widetilde{M}$. Let $\sigma(\mu)$ be the Weyl group representation attached to $\mu$ and $\tilde{\delta}$ (Definition 10.5). Then $\sigma(\mu)$ is irreducible, and $\mu$ corresponds to the pair $(\tilde{\delta}, \sigma(\mu))$.

Conversely, given a pair $(\tilde{\delta}, \sigma) \in S$, construct an irreducible representation $\tau$ of $\widetilde{M^{\prime}}$ by Lemma 10.2, Then $\tau$ extends uniquely to a genuine irreducible petite representation $\mu$ of $\widetilde{K}$.

Remark 11.2. A weaker version of Proposition 11.1 holds in the case of two root lengths, although the construction of $\mu$ is more complicated. (To begin with, $\mu$ need not restrict to a multiple of an irreducible representation of $\widetilde{M}$.) We give arguments for types $B_{n}$ and $C_{n}$ in Section 12 below.

Example 11.3. If $\sigma$ is trivial, then we recover the bijection between pseudospherical representations of $\widetilde{M}$ and $\widetilde{K}$ (Proposition 5.2 ).

Example 11.4. Let $G=\mathrm{SL}(3), \widetilde{G}=\widetilde{\mathrm{SL}}(3)$, so $K \simeq \mathrm{SO}(3)$ and $\widetilde{K} \simeq \mathrm{SU}(2)$. The groups $\widetilde{M}, \widetilde{M^{\prime}}$ and $W$ in (10.1) are the quaternion group of order 8 (cf. Proposition 5.2), the binary octahedral group of order 48 , and the symmetric group $S_{3}$, respectively. The binary octahedral group has three genuine irreducible representations $\tau_{+}, \tau_{-}$, and $\tau^{\prime}$, of dimensions 2,2 , and 4 respectively. Here $\tau_{+}$is the restriction to $\widetilde{M}^{\prime}$ of the two-dimensional representation of $\widetilde{K}$. The corresponding representation of $W$ is trivial (Lemma 10.2). The irreducible four-dimensional representation of $\widetilde{K}$ restricts to $\tau^{\prime}$ and corresponds to the reflection representation of $W$. The representation $\tau_{-}$of $\widetilde{M^{\prime}}$ corresponds to the sgn representation of $W$. Note that $\tau_{-}$is not the restriction of an irreducible representation of $\widetilde{K}$. 
Proof. First we show that the two maps are inverses of each other on the level of representations of $\widetilde{M^{\prime}}$. For one direction, suppose $\left(\mu, V_{\mu}\right)$ is a genuine representation of $\widetilde{K}$. By Lemma 5.1, $\mu$ restricts to a multiple of a genuine pseudospherical representation $\tilde{\delta}$ of $\widetilde{M}$, and

$$
V_{\mu} \simeq \operatorname{Hom}_{\widetilde{M}}\left(F_{\tilde{\delta}}, V_{\mu}\right) \otimes F_{\tilde{\delta}} \simeq V_{\mu}[\tilde{\delta}]^{*} \otimes F_{\tilde{\delta}}
$$

as representations of $\widetilde{M^{\prime}}$.

On the other hand, given $(\tilde{\delta}, \sigma)$ and defining $V_{\mu}=F_{\tilde{\delta}} \otimes E_{\sigma}$, we have

$$
V_{\mu}[\tilde{\delta}]^{*} \simeq \operatorname{Hom}_{\widetilde{M}}\left(F_{\tilde{\delta}}, F_{\tilde{\delta}} \otimes E_{\sigma}\right) \simeq E_{\sigma} .
$$

We are left with two things to show. First, given $(\tilde{\delta}, \sigma) \in S$, let $\tau$ be the irreducible representation of $\widetilde{M^{\prime}}$ from Lemma 10.2. We must show that $\tau$ extends uniquely to an irreducible petite representation $\mu$ of $\widetilde{K}$. Secondly, given an irreducible genuine petite representation $\mu$ of $K$, we must show that the representation $\sigma(\mu)$ of $W$ is petite.

For the first assertion, recall from (4.14) the element $\widetilde{\sigma}_{\alpha}=\exp \left(\pi Z_{\alpha} / 2\right) \in \widetilde{M}^{\prime}$. We have $\widetilde{\sigma}_{\alpha}^{2}=\widetilde{m}_{\alpha}$, and $\widetilde{\sigma}_{\alpha}$ is a representative in $\widetilde{M}^{\prime}$ for the reflection $s_{\alpha}$. Because $\alpha$ is metaplectic and $\tilde{\delta}$ is genuine, the possible eigenvalues of $\tau\left(\widetilde{m}_{\alpha}\right)$ are $\pm i$. Hence the possible eigenvalues of $\tau\left(\sigma_{\alpha}\right)$ are $\exp (i m \pi / 2)$, with $m= \pm 1 / 2$ or $\pm 3 / 2$. As in the proof of Proposition [5.2, if an extension $\mu$ of $\tau$ to $\widetilde{K}$ exists, then $d \mu\left(Z_{\alpha}\right)$ must act on the $\exp (i m \pi / 4)$ eigenspace of $\tau$ with eigenvalues $i(m+4 k)$, with $k$ integral. If in addition $\mu$ is petite, then (Definition 4.9) necessarily $|m+4 k| \leq 2$. This last requirement forces the integer $k$ to be zero. That is, $d \mu\left(Z_{\alpha}\right)$ must act on the $\exp (i m \pi / 2)$ eigenspace of $\tau\left(\widetilde{\sigma}_{\alpha}\right)$ by the scalar $i m$. Informally,

$$
d \mu\left(Z_{\alpha}\right)=2 \log \left(\tau\left(\widetilde{\sigma}_{\alpha}\right)\right) / \pi .
$$

This proves uniqueness. It remains to show that $d \mu$ is actually a representation of $\mathfrak{k}$ that exponentiates to $\widetilde{K}$. As in the proof of Proposition 5.2 this reduces to a rank two calculation. If $\left\langle\alpha, \beta^{\vee}\right\rangle=0$, then the corresponding three-dimensional subgroups commute with each other, so $\left[Z_{\alpha}, Z_{\beta}\right]=0, \widetilde{\sigma}_{\alpha}$ and $\widetilde{\sigma}_{\beta}$ commute, and the result is immediate.

Assume therefore that $\left\langle\alpha, \beta^{\vee}\right\rangle= \pm 1$. In this case the group $H$ defined in the proof of Proposition 5.2 is $\mathrm{SL}(3, \mathbb{R})$. This case was treated in Example 11.4. The petite hypothesis on $\sigma$ means that the restriction of $\sigma$ to the Weyl group of $H$ does not contain the sign representation. Therefore the restriction of $\tau$ to the binary octahedral group $\widetilde{M} \cap \widetilde{H}$ does not contain $\sigma_{-}$. The example therefore shows that $\tau$ extends uniquely to a petite representation of $\widetilde{K} \cap \widetilde{H}=\mathrm{SU}(2)$, a sum of two- and four-dimensional representations.

This completes the proof that $d \mu$ is a representation of $\mathfrak{k}$. That it exponentiates to $K$ can be proved as in Proposition 5.2

For the second assertion, suppose $\mu$ is a genuine petite $\widetilde{K}$-type. Then $\mu$ restricted to any rank 2 subgroup $\widetilde{H} \cap \widetilde{K}$ as above is a direct sum of the irreducible 2and 4-dimensional representations of SU(2). These correspond to the trivial and reflection representations of $S_{3}$ by Example 11.4, so the sign representation of $S_{3}$ does not appear. Therefore $\sigma(\mu)$ is a petite representation of $W$. This completes the proof. 


\section{Construction of petite $\widetilde{K}$-types: Classical groups}

In this section we enumerate petite representations for classical groups. A tilde over a simply connected split classical group (like $\widetilde{\operatorname{Spin}}(n+1, n))$ means the nonlinear double cover of Definition 1.1. The results for linear groups originated in Ba1]. One can find an improved and simplified account in Ba2, to which we will therefore also refer.

12.1. Petite $\widetilde{K}$-types for $\operatorname{SL}(n)$ and $\widetilde{\mathrm{SL}}(n)$. Let $G=\widetilde{\mathrm{SL}}(n)$, so $\widetilde{K}=\operatorname{Spin}(n)$. We parametrize the irreducible representations of $\operatorname{Spin}(n)$ by highest weights as usual. A typical highest weight is $\mu=\left(a_{1}, \ldots, a_{r}\right)$, with $a_{1} \geq \cdots \geq a_{r-1} \geq\left|a_{r}\right|$, with $r=[n / 2]$, and $a_{i} \in \mathbb{Z}$ for all $i$, or $a_{i} \in \mathbb{Z}+1 / 2$ for all $i$. If $n$ is odd, then $a_{r} \geq 0$.

For $a_{r} \geq 0$ and $\epsilon= \pm$, write $\left(a_{1}, \ldots, a_{r}\right)_{\epsilon}$ for the highest weight $\left(a_{1}, \ldots, a_{r-1}, \epsilon a_{r}\right)$ with the understanding that $\epsilon=+$ if $n$ is odd.

Let $\operatorname{Spin}_{\epsilon}$ be the irreducible representation with highest weight $(1 / 2, \ldots, 1 / 2)_{\epsilon}$. If $n$ is odd, only $\epsilon=+$ is allowed, and we write $\operatorname{Spin}=\operatorname{Spin}_{+}$.

Lemma 12.1 ([Ba1, $\mathrm{Ba} 2])$. Let $G=\mathrm{SL}(n, \mathbb{R})$. For $1 \leq k \leq n / 2$, the $K$-type $\mu_{k, \epsilon}$ with highest weight

$$
(\underbrace{2, \ldots, 2}_{k}, 0, \ldots)_{\epsilon}
$$

is petite. (Here $\epsilon=-$ is allowed only if $n$ is even, and $\mu_{k,+}=\mu_{k,-}$ unless $k=$ $n / 2$.) The corresponding $W$-representation (in the notation of Section 9.1 and Definition 10.5) is given by

$$
V_{\mu_{k, \epsilon}}[\mathbf{1}]=(n-k, k) .
$$

Moreoever, the $K$-types $\left\{\mu_{k, \epsilon} \mid 1 \leq k \leq[k / 2]\right\}$ detect spherical unitarity in the sense of Definition 13.11 below.

Now we consider $\widetilde{\mathrm{SL}}(n)$.

Lemma 12.2. Let $G=\widetilde{\mathrm{SL}}(n, \mathbb{R})$.

(1) Assume that $n$ is odd. Write $\widetilde{\delta}$ for the unique genuine pseudospherical representation of $\widetilde{M}$ arising as the restriction of the spin representations of $\widetilde{K}$; see Table 1, For $k \leq(n-1) / 2$, the $\widetilde{K}$-types with highest weights

$$
\mu_{k}=(\underbrace{3 / 2, \ldots, 3 / 2}_{k}, 1 / 2, \ldots, 1 / 2)
$$

exhaust the set $\Sigma_{\widetilde{K}}(\widetilde{G}, \tilde{\delta})$ of $\tilde{\delta}$-petite $\widetilde{K}$-types in the sense of Definition 10.7 , In the notation of Definition 10.5 ,

$$
V_{\mu_{k}}[\tilde{\delta}]=(n-k, k) .
$$

(2) Assume that $n$ is even. According to Table 1, there are two genuine pseudospherical representations $\tilde{\delta}_{ \pm}$; they are obtained (respectively) by restricting the two representations $\operatorname{Spin}_{ \pm}$to $\widetilde{M}$. For $k \leq n / 2$, the $\widetilde{K}$-types with highest weights

$$
\mu_{k, \epsilon}=(\underbrace{3 / 2, \ldots, 3 / 2}_{k}, 1 / 2, \ldots, 1 / 2)_{(-1)^{k} \epsilon}
$$


exhaust the set $\Sigma_{\widetilde{K}}\left(\widetilde{G}, \tilde{\delta}_{\epsilon}\right)$ of $\tilde{\delta}_{\epsilon}$-petite $\widetilde{K}$-types in the sense of Definition 10.7. In the notation of Definition 10.5,

$$
V_{\mu_{k, \epsilon}}\left[\tilde{\delta}_{\epsilon}\right]=(n-k, k) .
$$

Proof. The bijection between genuine petite $\widetilde{K}$-types and petite Weyl group representations is given by Proposition 11.1. The explicit calculation of this bijection follows from the methods of Ba1]. Except in a few cases the correspondence is determined by the dimensions.

Remark 12.3. Because we will encounter increasingly detailed statements of the form of Lemmas 12.1 and 12.2, it is useful to introduce some notation to make their statements more compact. We summarize the statement of Lemma 12.1 by saying that the following $K$-types are petite and detect spherical unitarity for $\operatorname{SL}(n, \mathbb{R})$ :

$$
\begin{array}{cc}
\mu & V_{\mu}[\mathbf{1}] \\
(\underbrace{2, \ldots, 2}_{k}, 0, \ldots)_{\epsilon} & (n-k, k) \quad k \leq n / 2
\end{array}
$$

Meanwhile, we summarize the statement of Lemma 12.2 by saying that the petite $\widetilde{K}$-types for $\widetilde{\mathrm{SL}}(n, \mathbb{R})$ and corresponding Weyl group representations are as follows:

$$
\begin{array}{ccclc}
\mu & \tilde{\delta} & V_{\mu}[\tilde{\delta}] & \\
(\underbrace{3 / 2, \ldots}_{k}, 1 / 2, \ldots)_{\epsilon} & \tilde{\delta}_{(-1)^{k} \epsilon} & (n-k, k) & k \leq n / 2 & n \text { even } \\
(\underbrace{3 / 2, \ldots}_{k}, 1 / 2, \ldots)_{+} & \tilde{\delta}_{+} & (n-k, k) & k \leq(n-1) / 2 & n \text { odd }
\end{array}
$$

12.2. Petite $\widetilde{K}$-types for $\operatorname{Sp}(2 n, \mathbb{R})$ and $\widetilde{\mathrm{Sp}}(2 n, \mathbb{R})$. Assuming the results of [Ba1, it is straightforward to prove the analogue of Proposition 11.1 for $\widetilde{\mathrm{Sp}}(2 n)$.

Let $K$ be a maximal compact subgroup of $\operatorname{Sp}(2 n, \mathbb{R})$, with inverse image $\widetilde{K}$ in $\widetilde{\operatorname{Sp}}(2 n, \mathbb{R})$. We identify the irreducible representations of $\widetilde{K}$ and $K$ with their highest weights. A highest weight may be written $\left(a_{1}, \ldots, a_{n}\right)$ with $a_{1} \geq \cdots \geq a_{n}$. Exponentiating to a weight for the torus in $\widetilde{K}$ means that either $a_{i} \in \mathbb{Z}$ for all $i$, or $a_{i} \in \mathbb{Z}+\frac{1}{2}$ for all $i$. The weight $(1, \ldots, 1)$ corresponds to the one-dimensional determinant character det of $K$.

Let $\mu_{ \pm}$be the genuine character of $\widetilde{K}$ with highest weight $\pm(1 / 2, \ldots, 1 / 2)$ or, equivalently, satisfying $\mu_{ \pm}^{2}=\operatorname{det}^{ \pm}$. Let $\tilde{\delta}_{ \pm}$be the restriction of $\mu_{ \pm}$to $\widetilde{M}$.

By Lemma 4.18 a $\widetilde{K}$-type $\mu=\left(a_{1}, \ldots, a_{n}\right)$ is petite if and only if $a_{1} \leq 2$, $a_{n} \geq-2$, and $a_{1}-a_{n} \leq 2$.

Lemma 12.4 ([Ba1], $\mathrm{Ba} 2])$. Retain the notation outlined in Remark 12.3, The following $K$-types for $\operatorname{Sp}(2 n, \mathbb{R})$ are petite and detect spherical unitarity:

$$
\begin{aligned}
& \mu \quad V_{\mu}[\mathbf{1}] \\
& (\underbrace{2, \ldots, 2}_{k}, 0, \ldots, 0) \quad(n-k) \times(k) \quad k \leq n \\
& (0, \ldots, 0, \underbrace{-2, \ldots,-2}_{k}) \quad(n-k) \times(k) \quad k \leq n \\
& (\underbrace{1, \ldots, 1}_{k}, 0, \ldots, 0, \underbrace{-1, \ldots,-1}_{k}) \quad(n-k, k) \quad k \leq n / 2
\end{aligned}
$$


Lemma 12.5. Retain the notation outlined in Remark 12.3 . Let $\widetilde{G}=\widetilde{\operatorname{Sp}}(2 n, \mathbb{R})$. The genuine petite $\widetilde{K}$-types and the associated Weyl group representations are as follows:

$$
\begin{aligned}
& \mu \quad \tilde{\delta} \quad V_{\mu}[\tilde{\delta}] \\
& (\underbrace{1 / 2, \ldots, 1 / 2}_{k},-3 / 2, \ldots,-3 / 2) \quad \tilde{\delta}_{+} \quad(n-k) \times(k) \\
& (3 / 2, \ldots, 3 / 2, \underbrace{-1 / 2, \ldots,-1 / 2}_{k}) \quad \tilde{\delta}_{-} \quad(n-k) \times(k) \\
& (\underbrace{3 / 2, \ldots, 3 / 2}_{k}, 1 / 2, \ldots, 1 / 2, \underbrace{-1 / 2, \ldots,-1 / 2}_{k}) \quad \tilde{\delta}_{+} \quad(n-k, k) \\
& (\underbrace{1 / 2, \ldots, 1 / 2}_{k},-1 / 2, \ldots,-1 / 2, \underbrace{-3 / 2, \ldots,-3 / 2}_{k}) \quad \tilde{\delta}_{-} \quad(n-k, k)
\end{aligned}
$$

Proof. Fix a genuine irreducible representation $\mu$ of $\widetilde{K}$. Then $\mu \otimes \tilde{\delta}_{ \pm}$factors to an irreducible representation of $K$, and

$$
\operatorname{Hom}_{\widetilde{M}}\left(\mu, \tilde{\delta}_{ \pm}\right) \simeq \operatorname{Hom}_{M}\left(\mu \otimes \tilde{\delta}_{\mp}, \tilde{\delta}_{ \pm} \otimes \tilde{\delta}_{\mp}\right) \simeq V_{\mu \otimes \tilde{\delta} \mp}[\mathbf{1}] .
$$

The result follows immediately from the preceding lemma.

Remark 12.6. In spite of the simplicity of this proof, note that the pseudospherical unitary dual of $\widetilde{\operatorname{Sp}}(2 n, \mathbb{R})$ is related to the spherical unitary dual of $\operatorname{Spin}(n+1, n)$, and not to that of $\operatorname{Sp}(2 n, \mathbb{R})$. See Section 14.

12.3. Petite $\widetilde{K}$-types for Spin and $\widetilde{\operatorname{Spin}}$. Let $\widetilde{G}=\widetilde{\operatorname{Spin}}(n, n)$, so $\widetilde{K} \simeq \operatorname{Spin}(n) \times$ $\operatorname{Spin}(n)$. See Section 12.1 for a discussion of highest weights for $\operatorname{Spin}(n)$.

A $\widetilde{K}$-type is given by a highest weight $\left(a_{1}, \ldots, a_{r}\right) \otimes\left(b_{1}, \ldots, b_{r}\right)$, with $r=[n / 2]$. This is genuine if $a_{i}-b_{j} \in \mathbb{Z}+\frac{1}{2}$ for all $i, j$.

Let $\mu(R, \pm)=\mathbf{1} \otimes \operatorname{Spin}_{ \pm}$, and $\mu(L, \pm)=\operatorname{Spin}_{ \pm} \otimes \mathbf{1}$; if $n$ is odd, write $\mu(R, \pm)=$ $\mathbf{1} \otimes$ Spin. (The letters $R$ and $L$ stand for "right" and "left".) Let $\tilde{\delta}(R, \pm)$ and $\tilde{\delta}(L, \pm)$ be the restrictions of $\mu(R, \pm)$ and $\mu(L, \pm)$ to $\widetilde{M}$. These are the genuine pseudospherical representations of $\widetilde{K}$ and $\widetilde{M}$.

Lemma 12.7 (Ba1, Ba2]). Retain the notation outlined in Remark 12.3. The $K$-types for $\operatorname{Spin}(n, n)$ listed in Table 4 are petite and detect spherical unitarity.

TABle 4. Petite $K$-types for $\operatorname{Spin}(n, n)$

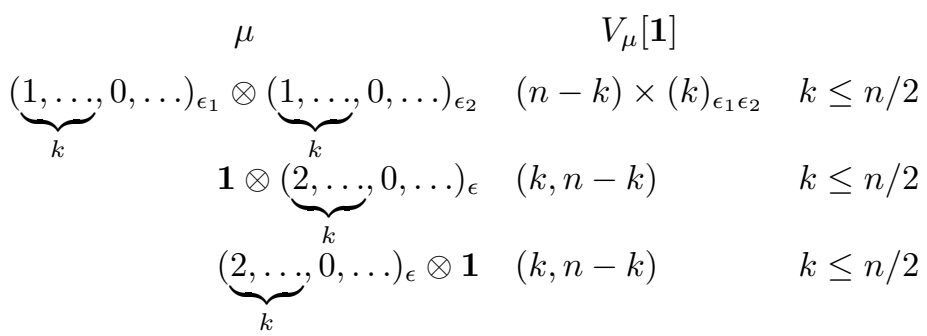


TABLE 5. Genuine petite $\widetilde{K}$-types for $\widetilde{\operatorname{Spin}}(n, n)$

$$
\begin{array}{cclc}
\mu & \tilde{\delta} & V_{\mu}[\tilde{\delta}] & \\
(\underbrace{1, \ldots}_{k}, 0, \ldots)_{\sigma} \otimes \operatorname{Spin}_{\epsilon} & \tilde{\delta}\left(R,(-1)^{k} \epsilon\right) & (k) \times(n-k)_{\epsilon \sigma} & k \leq n / 2 \\
\operatorname{Spin}_{\epsilon} \otimes(\underbrace{1, \ldots}_{k}, 0, \ldots)_{\sigma} & \tilde{\delta}\left(L,(-1)^{k} \epsilon\right) & (k) \times(n-k)_{\epsilon \sigma} & k \leq n / 2 \\
\mathbf{1} \otimes(\underbrace{3 / 2, \ldots,}_{k}, 1 / 2, \ldots)_{\epsilon} & \tilde{\delta}\left(R,(-1)^{k} \epsilon\right) & (n-k, k) & k \leq n / 2 \\
(\underbrace{3 / 2, \ldots, 1 / 2, \ldots)_{\epsilon} \otimes \mathbf{1}}_{k} & \tilde{\delta}\left(L,(-1)^{k} \epsilon\right) & (n-k, k) & k \leq n / 2
\end{array}
$$

Lemma 12.8. Retain the notation outlined in Remark 12.3 , Let $\widetilde{G}=\widetilde{\operatorname{Spin}}(n, n)$. The genuine petite $\widetilde{K}$-types and the associated Weyl group representations are as listed in Table 5 .

Proof. See the proof of Lemma 12.2.

Lemma 12.9 ([Ba1], $\mathrm{Ba} 2])$. Retain the notation outlined in Remark 12.3. The following $K$-types for $\operatorname{Spin}(n+1, n)$ are petite and detect spherical unitarity:

$$
\begin{aligned}
& \mu \quad V_{\mu}[\mathbf{1}] \\
& (\underbrace{1, \ldots, 1}_{k}, 0, \ldots, 0) \otimes(\underbrace{1, \ldots, 1}_{k}, 0, \ldots, 0)_{\epsilon} \quad(n-k) \times(k) \quad k \leq n / 2 \\
& (\underbrace{1, \ldots, 1}_{n+1-k}, 0, \ldots, 0)_{\epsilon} \otimes(\underbrace{1, \ldots, 1}_{n-k}, 0, \ldots, 0) \quad(n-k) \times(k) \quad(n+1) / 2 \leq k \leq n \\
& \mathbf{1} \otimes(\underbrace{2, \ldots, 2}_{k}, 0, \ldots, 0)_{\epsilon} \quad(n-k, k) \quad k \leq n / 2
\end{aligned}
$$

Now let $\widetilde{G}=\widetilde{\operatorname{Spin}}(n+1, n)$, so $\widetilde{K}=\operatorname{Spin}(n+1) \times \operatorname{Spin}(n)$. If $n$ is even, let $\mu_{ \pm}=\mathbf{1} \otimes \operatorname{Spin}_{ \pm}$and let $\tilde{\delta}_{ \pm}$be the restriction of $\mu_{ \pm}$to $\widetilde{M}$. If $n$ is odd, let $\mu=\mathbf{1} \otimes$ Spin and let $\tilde{\delta}$ be the restriction of $\mu$ to $\widetilde{M}$. These are the genuine pseudospherical representations of $\widetilde{K}$ and $\widetilde{M}$ (Table 2).

Lemma 12.10. Retain the notation outlined in Remark 12.3 , Let $\widetilde{G}=\widetilde{\operatorname{Spin}}(n+$ $1, n)$. The genuine petite $\widetilde{K}$-types and the associated Weyl group representations are as listed in Table 6 .

Proof. If $n$ is odd, the restriction of any genuine $\widetilde{K}$-type to $\widetilde{M}$ is a multiple of $\tilde{\delta}$ by Proposition [5.6. If $n$ is even, $\tilde{\delta}_{ \pm}$have the same central character, so $\mu$ is not necessarily isotypic for $\widetilde{M}$. We have the following cases.

(1) $\mu=\mathbf{1} \otimes(\underbrace{3 / 2, \ldots, 1 / 2}_{k}, \ldots)_{\epsilon}, n$ even. In this case $\mu$ restricts irreducibly to $\operatorname{Spin}(n) \times \operatorname{Spin}(n)$. By Lemma 12.8, $V_{\mu}[\tilde{\delta}]$ is an irreducible representation of $W\left(B_{n}\right)$ whose restriction to $W\left(D_{n}\right)$ is $(n-k, k)$. The fact that $\tilde{\delta}$ is as stated can be deduced by tensoring $\mu$ with the pseudospherical representation $\mu_{ \pm}$and comparing the result with the list of petite representations of $\operatorname{Spin}(n+1, n)$ in Lemma 12.9, the details are as in case (4) below. 
TABLE 6. Genuine petite $\widetilde{K}$-types for $\widetilde{\operatorname{Spin}}(n+1, n)$

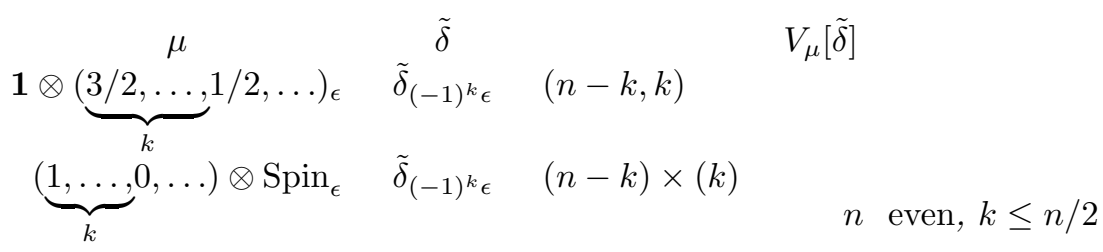

$$
\begin{aligned}
& (\underbrace{1, \ldots, 0}_{k}, \ldots) \otimes \operatorname{Spin}_{\epsilon} \quad \tilde{\delta}_{(-1)^{k+1} \epsilon} \quad(k-1) \times(n-k+1) \quad n \text { even, } k \leq n / 2 \\
& \begin{array}{r}
(\underbrace{1, \ldots,}_{k}, \ldots) \otimes \operatorname{Spin} \quad \tilde{\delta} \quad(n-k) \times(k) \oplus(k-1) \times(n-k+1) \\
n \text { odd, } k \leq(n-1) / 2
\end{array} \\
& (1, \ldots, \pm 1) \otimes \operatorname{Spin} \quad \tilde{\delta} \quad((n-1) / 2) \times((n+1) / 2) \quad n \text { odd }
\end{aligned}
$$

(2) $\mu=(\underbrace{1, \ldots,}_{k} 0, \ldots) \otimes$ Spin, $n$ odd, $k \leq(n-1) / 2$. In order to compute $V_{\mu}[\tilde{\delta}]$ as a $W$ representation, write $\mu_{L}=(1, \ldots, 0, \ldots)$ (with $k$ ones), and use the isomorphism

$$
\operatorname{Hom}_{\widetilde{M}}\left(\tilde{\delta}, \mu_{L} \otimes \operatorname{Spin}\right) \simeq \operatorname{Hom}_{M}\left(\mathbf{1}, \mu_{L} \otimes\left(\operatorname{Spin} \otimes \operatorname{Spin}^{*}\right)\right) .
$$

It is well known and easy to check that

$$
\text { Spin } \otimes \text { Spin }=\sum_{0 \leq m<n / 2} \bigwedge^{m}\left(\mathbb{C}^{n}\right)
$$

The highest weights of these representations are

$$
\bigwedge^{m}\left(\mathbb{C}^{n}\right) \leftrightarrow(\underbrace{1, \ldots,}_{m} 0, \ldots) \quad(m<n / 2) .
$$

The conclusion from these calculations is that

$$
V_{\mu}[\tilde{\delta}] \simeq \sum_{m<n / 2}(\underbrace{1, \ldots,}_{k} 0, \ldots) \otimes(\underbrace{1, \ldots,}_{m} 0, \ldots)[\mathbf{1}] .
$$

The summand on the right side has been computed in Lemma 12.9 in case $m=$ $k$ or $m=k-1$; these give the Weyl group representations $(n-k) \times(k)$ and $(k-1) \times(n-k+1)$. The dimensions of these two representations add up to $\left(\begin{array}{c}n+1 \\ k\end{array}\right)$, which is easily computed to be the dimension of $V_{\mu}[\tilde{\delta}]$; so the other summands must be zero.

(3) $(1, \ldots, \pm 1) \otimes$ Spin, $n$ odd. This is almost identical to case (2).

(4) $(\underbrace{1, \ldots,}_{k}, \ldots) \otimes \operatorname{Spin}_{\epsilon}, n$ even. We begin as in (2), computing

$$
\operatorname{Hom}_{\widetilde{M}}\left(\tilde{\delta}_{\gamma}, \mu_{L} \otimes \operatorname{Spin}_{\epsilon}\right) \simeq \operatorname{Hom}_{M}\left(\mathbf{1}, \mu_{L} \otimes \operatorname{Spin}_{\epsilon} \otimes \operatorname{Spin}_{\gamma}^{*}\right) .
$$

It is straightforward to see

$$
\operatorname{Spin}_{\epsilon} \otimes \operatorname{Spin}_{\gamma}^{*} \simeq E \oplus \sum \bigwedge^{k}\left(\mathbb{C}^{n}\right),
$$


where the sum is taken over $0 \leq m<n / 2, k \equiv \epsilon \gamma(\bmod 2)$. The "error term" $E$ appears if and only if $n / 2 \equiv \epsilon \gamma(\bmod 2)$; in that case it is one of the two summands of $\bigwedge^{n / 2} \mathbb{C}^{n}$ and has highest weight of the form $(1, \ldots, 1)_{\phi}$. The argument from this point is as in (2), using Lemma 12.9.

This completes the proof.

From Proposition 11.1 and the tables we conclude:

Proposition 12.11. Suppose $G$ is a classical group or is simply laced, and $\tilde{\delta}$ is an irreducible pseudospherical representation of $\widetilde{M}$. Then $\Sigma_{W}(\widetilde{G}, \tilde{\delta})$ (Definition 10.7) is the set of all petite representations of $W$.

\section{INTERTWINING OPERATOR METHODS}

We begin with a formal construction. Recall that $\Delta$ is the root system of $G, R$ the root lattice, and $\Delta^{+}$the set of positive roots corresponding to $B$. Write $S$ for the set of simple roots.

Recall Definition 4.4. We define a map $\tau: \Delta \rightarrow\{1,2\}$ by

$$
\tau(\alpha)= \begin{cases}2 & \text { if } \alpha \text { is metaplectic in } \widetilde{G}, \\ 1 & \text { if } \alpha \text { is not metaplectic in } \widetilde{G}\end{cases}
$$

We now fix

$$
\nu \in \mathfrak{a}_{\mathbb{C}}^{*}, \quad \nu^{\ell}=d \Psi^{t}(\nu) \in\left(\mathfrak{a}^{\ell}\right)_{\mathbb{C}}^{*}
$$

The characteristic property 6.2 of $\Psi$ implies that

$$
\tau(\alpha)\left\langle\nu, \alpha^{\vee}\right\rangle=\left\langle\nu^{\ell},\left(\alpha^{\ell}\right)^{\vee}\right\rangle \quad(\alpha \in \Delta) .
$$

We now introduce a series of linear operators related to intertwining operators. For the first, suppose $(\sigma, E)$ is a representation of the Weyl group $W$, and $\alpha \in \Delta$. The reflection $s_{\alpha}$ has order 2 , so it acts in $E$ with eigenvalues +1 and -1 . We write

$$
E=E(+, \alpha) \oplus E(-, \alpha)
$$

accordingly. We now define

$$
A\left(s_{\alpha}, \sigma, \nu^{\ell}\right)=\left(1+\left\langle\nu^{\ell}, \alpha^{\vee}\right\rangle\right)^{-1}\left(I+\left\langle\nu^{\ell}, \alpha^{\vee}\right\rangle \sigma\left(s_{\alpha}\right)\right) .
$$

This makes sense as long as $\left\langle\nu^{\ell}, \alpha^{\vee}\right\rangle \neq-1$. A little more explicitly,

$$
A\left(s_{\alpha}, \sigma, \nu^{\ell}\right) v=\frac{1+\epsilon\left\langle\nu, \alpha^{\vee}\right\rangle}{1+\left\langle\nu, \alpha^{\vee}\right\rangle} v \quad(v \in E(\epsilon, \alpha)) .
$$

Similarly, define

$$
\widetilde{A}\left(s_{\alpha}, \sigma, \nu\right)=\left(1+\tau(\alpha)\left\langle\nu, \alpha^{\vee}\right\rangle\right)^{-1}\left(I+\tau(\alpha)\left\langle\nu, \alpha^{\vee}\right\rangle \sigma\left(s_{\alpha}\right)\right) .
$$

This makes sense as long as $\tau(\alpha)\left\langle\nu, \alpha^{\vee}\right\rangle \neq-1$. Explicitly,

$$
\widetilde{A}\left(s_{\alpha}, \sigma, \nu\right) v=\frac{1+\epsilon \tau(\alpha)\left\langle\nu, \alpha^{\vee}\right\rangle}{1+\tau(\alpha)\left\langle\nu, \alpha^{\vee}\right\rangle} v \quad(v \in E(\epsilon, \alpha)) .
$$

Suppose now that $w \in W$ is any element. Choose a reduced decomposition

$$
w=s_{\alpha_{n}} s_{\alpha_{n-1}} \cdots s_{\alpha_{1}},
$$

where each $\alpha_{i} \in S$. For $1 \leq i \leq n$ define $w^{(i)}=s_{\alpha_{i}} s_{\alpha_{i-1}} \cdots s_{\alpha_{1}}$, and set $w^{(0)}=1$. 
Define

$$
A\left(w, \sigma, \nu^{\ell}\right)=\prod_{i=1}^{n} A\left(s_{\alpha_{i}}, \sigma, w^{(i-1)} \nu^{\ell}\right)
$$

and

$$
\widetilde{A}(w, \sigma, \nu)=\prod_{i=1}^{n} \widetilde{A}\left(s_{\alpha_{i}}, \sigma, w^{(i-1)} \nu\right) .
$$

These linear operators are defined for $\nu^{\ell}$ and $\nu$ not belonging to $n$ hyperplanes. Explicitly, write

$$
\Delta^{+}(w)=\left\{\beta \in \Delta^{+} \mid w^{-1}(\beta) \in \Delta^{-}\right\} .
$$

This is a set of $n$ positive roots, and it turns out that

$$
\Delta^{+}(w)=\left\{\left(w^{(i-1)}\right)^{-1}\left(\alpha_{i}\right) \mid 1 \leq i \leq n\right\}
$$

It follows that $A\left(w, \sigma, \nu^{\ell}\right)$ is defined as long as

$$
\left\langle\nu^{\ell}, \beta^{\vee}\right\rangle \neq-1, \quad \beta \in \Delta^{+}(w) .
$$

Similarly, $\widetilde{A}(w, \sigma, \nu)$ is defined as long as

$$
\tau(\beta)\left\langle\nu, \beta^{\vee}\right\rangle \neq-1, \quad \beta \in \Delta^{+}(w) .
$$

In particular, the operators are defined whenever $\nu^{\ell}$ and $\nu$ have dominant real part. If $w_{0}$ is the long element of $W$, we will be particularly interested in the operators

$$
A\left(\sigma, \nu^{\ell}\right)=A\left(w_{0}, \sigma, \nu^{\ell}\right), \quad \widetilde{A}(\sigma, \nu)=\widetilde{A}\left(w_{0}, \sigma, \nu\right)
$$

Our notation implies that $A\left(w, \sigma, \nu^{\ell}\right)$ is independent of the choice of reduced decomposition of $w$ in $(13.3)(\mathrm{a})$. This is true and not very difficult to prove, but since we will make no use of the fact, we omit the argument. We are interested in these operators because of their relationship to intertwining operators, and we are interested in intertwining operators because they can be used to construct invariant Hermitian forms on representations. A matrix like $A\left(\sigma, \nu^{\ell}\right)$ will turn out to be part of the matrix of an invariant Hermitian form. To see that this makes sense, we need to know that $A\left(\sigma, \nu^{\ell}\right)$ is (sometimes) a self-adjoint operator. (Because $E$ is the space of the unitary Weyl group representation $\sigma$, it carries a natural Hilbert space structure; this is what defines adjoints for operators on E.) Because

$$
\sigma\left(s_{\alpha}\right)^{*}=\sigma\left(s_{\alpha}\right)^{-1}=\sigma\left(s_{\alpha}\right)
$$

we get immediately

$$
A\left(s_{\alpha}, \sigma, \nu^{\ell}\right)^{*}=A\left(s_{\alpha}, \sigma, \bar{\nu}^{\ell}\right)=A\left(s_{\alpha}, \sigma,-s_{\alpha}\left(\bar{\nu}^{\ell}\right)\right),
$$

and similarly for $\widetilde{A}$. An easy calculation with (13.3) gives

$$
A\left(w, \sigma, \nu^{\ell}\right)^{*}=A\left(w^{-1}, \sigma,-w\left(\bar{\nu}^{\ell}\right)\right), \quad \widetilde{A}(w, \sigma, \nu)^{*}=\widetilde{A}\left(w^{-1}, \sigma,-w(\bar{\nu})\right) .
$$

In particular, $A\left(\sigma, \nu^{\ell}\right)$ is self-adjoint if $w_{0}\left(\nu^{\ell}\right)=-\bar{\nu}^{\ell}$.

Lemma 13.7. Suppose $\nu \in \mathfrak{a}_{\mathbb{C}}^{*}$ and $\nu^{\ell}=d \Psi^{t}(\nu) \in\left(\mathfrak{a}^{\ell}\right)_{\mathbb{C}}^{*}$. Then for every representation $\sigma$ of $W$, and every element $w \in W$, we have

$$
\widetilde{A}(w, \sigma, \nu)=A\left(w, \sigma, \nu^{\ell}\right) \text {. }
$$

Proof. This is immediate from (13.1), (13.2), and (13.3). 
We turn now to a description of standard intertwining operators for pseudospherical principal series representations. We need these both for the linear group $G^{\ell}$ and for the nonlinear group $\widetilde{G}$. It is therefore convenient to generalize for a moment to the setting of Definition 4.1] so that $\widetilde{G}$ is a (possibly trivial) two-fold cover of a linear group $G$. Fix a pseudospherical representation $\tilde{\delta}$ of $\widetilde{M}$, and a weight $\nu \in \mathfrak{a}_{\mathbb{C}}^{*}$.

For each $w \in W$ there is a meromorphic family of standard intertwining operators

$$
T(w, \tilde{\delta}, \nu): I(\tilde{\delta}, \nu) \rightarrow I(w \tilde{\delta}, w \nu),
$$

which we normalize by requiring that they act as the identity on the unique pseudospherical $\widetilde{K}$-type $\mu_{0}$. Because $\tilde{\delta}$ is pseudospherical, $w \tilde{\delta}$ is equivalent to $\tilde{\delta}$ (Lemma 4.11); but writing the codomain in this form is convenient for thinking about generalizations to non-pseudospherical principal series. These operators are analytic at least for $\nu$ having dominant real part; the precise location of the poles will be clear from Lemma 13.13. We will be particularly interested in the long intertwining operator

$$
T(\tilde{\delta}, \nu)=T\left(w_{0}, \tilde{\delta}, \nu\right),
$$

whose image (when the real part of $\nu$ is dominant) is $J(\tilde{\delta}, \nu)$. Fix a $\widetilde{K}$-type $\mu$. By 10.4)(a), the intertwining operators induce maps

$$
T(w, \mu, \tilde{\delta}, \nu) \in \operatorname{Hom}\left(V_{\mu}[\tilde{\delta}], V_{\mu}[w \tilde{\delta}]\right) .
$$

Since we are realizing $\tilde{\delta}$ on the space of the pseudospherical $\widetilde{K}$-type $\mu_{0}$, we get natural identifications

$$
V_{\mu}[\tilde{\delta}]=\operatorname{Hom}_{\widetilde{M}}\left(V_{\mu}, V_{\mu_{0}}\right)=V_{\mu}[w \tilde{\delta}] .
$$

These identifications give

$$
T(w, \mu, \tilde{\delta}, \nu) \in \operatorname{End}\left(V_{\mu}[\tilde{\delta}]\right) .
$$

This space inherits a Hilbert space structure from $V_{\mu}$ and $V_{\mu_{0}}$. In analogy with (13.6), one can calculate easily that

$$
T(w, \mu, \tilde{\delta}, \nu)^{*}=T\left(w^{-1}, \mu, w \tilde{\delta},-\bar{\nu}\right) .
$$

If $w_{0} \nu=-\bar{\nu}$, it follows that $T(\mu, \tilde{\delta}, \nu)$ is self-adjoint. These self-adjoint operators define the invariant Hermitian forms on the spherical representations, giving the following classical and fundamental result.

Theorem 13.10. Suppose that $\nu \in \mathfrak{a}^{*}$ is real and dominant. Then $J(\tilde{\delta}, \nu)$ admits a nonzero invariant Hermitian form if and only if $w_{0} \nu=-\nu$. When the form exists, it is definite if and only if $T(\mu, \tilde{\delta}, \nu)$ is positive semi-definite for all $\widetilde{K}$-types $\mu$.

If $G$ is a linear group, this is standard; see for example [Kn, Chapter VII] or Ba1. The same proof holds in the nonlinear case with minor modifications.

Suppose now that $\Sigma$ is a collection of irreducible representations of $\widetilde{K}$.

Definition 13.11. Suppose that $J(\delta, \nu)$ is an irreducible Hermitian pseudospherical representation of $\widetilde{G}$; normalize its invariant Hermitian form to be positive on the pseudospherical $\widetilde{K}$-type $\mu_{0}$. We say $J(\tilde{\delta}, \nu)$ is unitary on $\Sigma$ if its invariant form is positive semi-definite on the $\mu$-isotypic component of $J(\tilde{\delta}, \nu)$ for all $\mu \in \Sigma$.

We say $\Sigma$ detects unitarity for $\tilde{\delta}$ if each Hermitian pseudospherical representation $J(\tilde{\delta}, \nu)$ is unitary if and only if it is unitary on $\Sigma$. We say $\Sigma$ detects spherical unitarity if it detects unitarity for the trivial character $\tilde{\delta}=\mathbf{1}$. 
To say that $\Sigma$ detects unitarity for $\tilde{\delta}$ means that for each nonunitary but Hermitian representation $J(\tilde{\delta}, \nu)$ (with $\nu$ dominant real), there is a $\mu \in \Sigma$ so that the operator $T(\mu, \tilde{\delta}, \nu)$ has a strictly negative eigenvalue.

The operator $T(w, \tilde{\delta}, \nu)$ has a factorization analogous to 13.3$)(\mathrm{b})$ :

$$
T(w, \tilde{\delta}, \nu)=\prod_{i=1}^{n} T\left(s_{\alpha_{i}}, w^{(i-1)} \tilde{\delta}, w^{(i-1)} \nu\right) ;
$$

here we are using a reduced decomposition of $w$ exactly as in 13.3$)(\mathrm{b})$. This induces a factorization

$$
T(w, \tilde{\delta}, \mu, \nu)=\prod_{i=1}^{n} T\left(s_{\alpha_{i}}, \mu, w^{(i-1)} \tilde{\delta}, w^{(i-1)} \nu\right) .
$$

Each factor $T\left(s_{\alpha_{i}}, w^{(i-1)} \tilde{\delta}, w^{(i-1)} \nu\right)$ is induced from an operator on a principal series representation of (essentially) $\mathrm{SL}(2, \mathbb{R})$ or $\widetilde{\mathrm{SL}}(2, \mathbb{R})$; so we calculate that operator first.

Recall from (4.12) that

$$
\operatorname{Ad}(\widetilde{m}) Z_{\alpha}= \pm Z_{\alpha} \text { for all } \widetilde{m} \in \widetilde{M} \text {. }
$$

For $k \in \frac{1}{2} \mathbb{Z}$, define

$$
V_{\mu}(k)=\left\{v \in V_{\mu} \mid d \mu\left(i Z_{\alpha}\right)(v)=k v\right\} .
$$

For $k \geq 0$, we will also use

$$
V_{\mu}( \pm k)=V_{\mu}(k)+V_{\mu}(-k) .
$$

Recall $\widetilde{m}_{\alpha}=\exp \left(\pi Z_{\alpha}\right)$. Its square $\widetilde{m}_{\alpha}^{2}$ is equal to the central element -1 if $\alpha$ is metaplectic, and to 1 if $\alpha$ is not metaplectic (see the discussion after (4.6)). It follows that $V_{\mu}( \pm k)$ is zero unless $k \in \mathbb{Z}+\frac{1}{2}$ if $\alpha$ is metaplectic, or $k \in \mathbb{Z}$ otherwise. Thus (writing $\frac{1}{2} \mathbb{Z}_{+}=\{0,1 / 2,1, \ldots\}$ ) we have

$$
V_{\mu}=\sum_{k \in \frac{1}{2} \mathbb{Z}_{+}} V_{\mu}( \pm k)
$$

an $\widetilde{M}$-invariant decomposition of $V_{\mu}$. Recalling that we are realizing $\tilde{\delta}$ on the pseudospherical $\widetilde{K}$-type $\mu_{0}$, this gives

$$
V_{\mu}[\delta] \simeq \bigoplus_{k \in \frac{1}{2} \mathbb{Z}_{+}} \operatorname{Hom}_{\widetilde{M}}\left(V_{\mu}( \pm k), V_{\mu_{0}}\right) .
$$

The summands satisfy

$$
\operatorname{Hom}_{\widetilde{M}}\left(V_{\mu}( \pm k), V_{\mu_{0}}\right)=0\left\{\begin{array}{l}
\text { if } \alpha \text { is metaplectic and } k \notin \mathbb{Z}+\frac{1}{2}, \text { or } \\
\text { if } \alpha \text { is not metaplectic and } k \notin 2 \mathbb{Z} .
\end{array}\right.
$$

Lemma 13.13. The intertwining operator $T\left(s_{\alpha}, \mu, \tilde{\delta}, \nu\right)$ acts on

$$
\operatorname{Hom}_{\widetilde{M}}\left(V_{\mu}( \pm k), V_{\mu_{0}}\right) \quad(k \geq 0)
$$

by 1 if $k=0$ or $k=1 / 2$, and for $k \geq 3 / 2$ by the scalar:

$$
\prod_{0 \leq j \leq[(k-1) / 2]} \frac{(k-1-2 j)-\left\langle\nu, \alpha^{\vee}\right\rangle}{(k-1-2 j)+\left\langle\nu, \alpha^{\vee}\right\rangle},
$$


with the product running over integer values of $j$. In more detail,

$$
\frac{1-\left\langle\nu, \alpha^{\vee}\right\rangle}{1+\left\langle\nu, \alpha^{\vee}\right\rangle} \cdot \frac{3-\left\langle\nu, \alpha^{\vee}\right\rangle}{3+\left\langle\nu, \alpha^{\vee}\right\rangle} \cdots \frac{(k-1)-\left\langle\nu, \alpha^{\vee}\right\rangle}{(k-1)+\left\langle\nu, \alpha^{\vee}\right\rangle} \quad k \geq 2 \text { even, }
$$

$$
\begin{array}{ll}
\frac{1 / 2-\left\langle\nu, \alpha^{\vee}\right\rangle}{1 / 2+\left\langle\nu, \alpha^{\vee}\right\rangle} \cdot \frac{5 / 2-\left\langle\nu, \alpha^{\vee}\right\rangle}{5 / 2+\left\langle\nu, \alpha^{\vee}\right\rangle} \cdots \frac{(k-1)-\left\langle\nu, \alpha^{\vee}\right\rangle}{(k-1)+\left\langle\nu, \alpha^{\vee}\right\rangle} & k \in \frac{3}{2}+2 \mathbb{Z}_{+}, \\
\frac{3 / 2-\left\langle\nu, \alpha^{\vee}\right\rangle}{3 / 2+\left\langle\nu, \alpha^{\vee}\right\rangle} \cdot \frac{7 / 2-\left\langle\nu, \alpha^{\vee}\right\rangle}{7 / 2+\left\langle\nu, \alpha^{\vee}\right\rangle} \cdots \frac{(k-1)-\left\langle\nu, \alpha^{\vee}\right\rangle}{(k-1)+\left\langle\nu, \alpha^{\vee}\right\rangle} & k \in \frac{5}{2}+2 \mathbb{Z}_{+} .
\end{array}
$$

The lemma is proved by a calculation in $\operatorname{SL}(2)$, which we omit.

We can decompose $V_{\mu_{0}}$ according to eigenspaces of $\mathbb{Z}_{\alpha}$ just as we did $V_{\mu}$. The assumption that $V_{\mu_{0}}$ is pseudospherical means that only $V_{\mu_{0}}(0)$ and $V_{\mu_{0}}( \pm 1 / 2)$ can be nonzero. Then

$$
V_{\mu}[\delta]=\sum_{k, \ell \in \frac{1}{2} \mathbb{Z}_{+}} \operatorname{Hom}_{\widetilde{M}}\left(V_{\mu}( \pm k), V_{\mu_{0}}( \pm \ell)\right)
$$

The element $m_{\alpha}$ acts by the scalar $i^{2 k}$ on $V_{\mu}(k)$ and on $V_{\mu_{0}}(k)$. Therefore an element $\phi$ of $\operatorname{Hom}_{\widetilde{M}}\left(V_{\mu}, V_{\mu_{0}}\right)$ must satisfy

$$
\phi\left(V_{\mu}(k)\right) \subset \sum_{k-\ell \in 2 \mathbb{Z}} V_{\mu_{0}}(\ell) .
$$

Since $\exp \left(\pi Z_{\alpha} / 2\right)$ is a representative of $s_{\alpha} \in W$, we can now calculate that in the representation $\sigma$ of $W$ on $V_{\mu}[\delta], \sigma\left(s_{\alpha}\right)$ acts on $\operatorname{Hom}_{\widetilde{M}}\left(V_{\mu}( \pm k), V_{\mu_{0}}\right)$ by the scalar

$$
\sigma\left(s_{\alpha}\right)= \begin{cases}(-1)^{k / 2} & (k \in 2 \mathbb{Z}), \\ (-1)^{(k-1 / 2) / 2} & \left(k \in \frac{1}{2}+2 \mathbb{Z}_{+}\right), \\ (-1)^{(k+1 / 2) / 2} & \left(k \in \frac{3}{2}+2 \mathbb{Z}_{+}\right) .\end{cases}
$$

More explicitly,

$$
\sigma\left(s_{\alpha}\right)= \begin{cases}+1 & \text { on } \operatorname{Hom}_{\widetilde{M}}\left(V_{\mu}(0), V_{\mu_{0}}\right), \\ -1 & \text { on } \operatorname{Hom}_{\widetilde{M}}\left(V_{\mu}( \pm 2), V_{\mu_{0}}\right), \\ +1 & \text { on } \operatorname{Hom}_{\widetilde{M}}\left(V_{\mu}( \pm 1 / 2), V_{\mu_{0}}\right), \\ -1 & \text { on } \operatorname{Hom}_{\widetilde{M}}\left(V_{\mu}( \pm 3 / 2), V_{\mu_{0}}\right) .\end{cases}
$$

Proposition 13.18. Suppose $\tilde{\delta}$ is pseudospherical, $\mu$ is petite, and

$$
\phi \in \operatorname{Hom}_{\widetilde{M}}\left(V_{\mu}, V_{\mu_{0}}\right)
$$

is an eigenvector of $\sigma\left(s_{\alpha}\right)$. Then

$$
T\left(s_{\alpha}, \mu, \tilde{\delta}, \nu\right) \phi= \begin{cases}\phi & \sigma\left(s_{\alpha}\right)(\phi)=\phi, \\ \frac{1-\left\langle\nu, \alpha^{\vee}\right\rangle}{1+\left\langle\nu, \alpha^{\vee}\right\rangle} \phi & \sigma\left(s_{\alpha}\right)(\phi)=-\phi, \alpha \text { not metaplectic, } \\ \frac{1 / 2-\left\langle\nu, \alpha^{\vee}\right\rangle}{1 / 2+\left\langle\nu, \alpha^{\vee}\right\rangle} \phi & \sigma\left(s_{\alpha}\right)(\phi)=-\phi, \alpha \text { metaplectic. }\end{cases}
$$

In particular, the action of $T\left(s_{\alpha}, \mu, \tilde{\delta}, \nu\right)$ on $V_{\mu}[\delta]$ is determined by the Weyl group representation $\sigma$.

Proof. This follows from 13.17)(b and Lemma 13.13 
Corollary 13.19. Suppose $\tilde{\delta}$ is a pseudospherical representation of $\widetilde{M}$, and $\mu$ is a petite representation of $\widetilde{K}$. Let $\sigma$ be the representation of $W$ on $V_{\mu}[\tilde{\delta}]$, and suppose $w \in W$. If $\widetilde{G}$ is the trivial cover of the linear group $G$, then

$$
T(w, \mu, \tilde{\delta}, \nu)=A(w, \sigma, \nu) .
$$

If $\widetilde{G}$ is the nonlinear double cover of a simply connected group, and $\tilde{\delta}$ is a genuine pseudospherical representation of $\widetilde{M}$, then

$$
T(w, \mu, \tilde{\delta}, \nu)=\widetilde{A}(w, \sigma, \nu) .
$$

Proof. This follows from corresponding identities for $T\left(s_{\alpha}, \mu, \tilde{\delta}, \nu\right)$ and $A\left(s_{\alpha}, \sigma, \nu\right)$ (cf. (13.2)(a) and Proposition 13.18) and the factorizations (13.3)(b) and (13.12).

Now suppose we are in the setting of Conjecture 7.1. In particular we have fixed a pseudospherical representation $\tilde{\delta}$ for $\widetilde{G}$. We write $K^{\ell}, A^{\ell}$, etc. for objects associated to $G^{\ell}$. We restrict consideration to real infinitesimal characters, so fix $\nu \in \mathfrak{a}_{0}^{*}$ and let $\nu^{\ell}=d \Psi^{t}(\nu)$.

Proposition 13.21. Suppose $\Sigma_{W}(\widetilde{G}, \tilde{\delta})=\Sigma_{W}\left(G^{\ell}, \mathbf{1}\right)$ (Definition 10.7). Then $J(\tilde{\delta}, \nu)$ is unitary on $\Sigma_{\widetilde{K}}(\widetilde{G}, \tilde{\delta})$ (Definition 13.11) if and only if $J\left(\nu^{\ell}\right)$ is unitary on $\Sigma_{K^{\ell}}\left(G^{\ell}, \mathbf{1}\right)$.

Proof. Suppose $J(\tilde{\delta}, \nu)$ is unitary on $\Sigma_{\widetilde{K}}(\widetilde{G}, \tilde{\delta})$. Then $\widetilde{A}(\sigma, \nu)$ is positive semidefinite for all $\sigma \in \Sigma_{W}(\widetilde{G})$ by 13.20$)(\mathrm{a})$. By Lemma 13.7. $A\left(\sigma, \nu^{\ell}\right)$ is positive semi-definite for all $\sigma \in \Sigma_{W}(\widetilde{G})=\Sigma_{W}\left(G^{\ell}\right)$. By 13.20$)\left(\mathrm{b}, J\left(\nu^{\ell}\right)\right.$ is unitary on $\Sigma_{K^{\ell}}\left(G^{\ell}, \mathbf{1}\right)$. The reverse direction is identical.

Corollary 13.22. Recall the notation of Conjecture 7.1. Assume that $\Sigma_{W}(\widetilde{G}, \tilde{\delta})=$ $\Sigma_{W}\left(G^{\ell}\right)$ (Definition 10.7).

If $\Sigma_{K^{\ell}}\left(G^{\ell}, \mathbf{1}\right)$ detects spherical unitarity for $G^{\ell}$ (Definition 13.11), then

$$
d \Psi^{t}(\mathbf{C S}(\tilde{\delta}, \widetilde{G})) \subset \mathbf{C S}\left(G^{\ell}\right) .
$$

If $\Sigma_{\widetilde{K}}(\widetilde{G}, \tilde{\delta})$ detects unitarity for $\tilde{\delta}$, then

$$
\mathbf{C S}\left(G^{\ell}\right) \subset d \Psi^{t}(\mathbf{C S}(\delta, \widetilde{G})) .
$$

Proof. Assume $\Sigma_{K^{\ell}}\left(G^{\ell}, \mathbf{1}\right)$ detects spherical unitarity for $G^{\ell}$, and suppose $J(\tilde{\delta}, \nu)$ is unitary. by the proposition, $J\left(\nu^{\ell}\right)$ is unitary on $\Sigma_{K^{\ell}}\left(G^{\ell}, \mathbf{1}\right)$, and therefore unitary since $\Sigma_{K^{\ell}}\left(G^{\ell}, \mathbf{1}\right)$ detects spherical unitarity. The other direction is identical.

Here is the deep result that gives substance to these elementary calculations.

Theorem 13.23 ([Ba1]). If $G$ is a classical linear group, then $\Sigma_{K}(G, \mathbf{1})$ detects spherical unitarity.

By Lemma 10.9 and Proposition 12.11, $\Sigma_{W}(\widetilde{G}, \tilde{\delta})=\Sigma_{W}\left(G^{\ell}, \mathbf{1}\right)$, so by Corollary 13.22 and Theorem 13.23 we conclude:

Theorem 13.24. Retain the notation of Conjecture 7.1 and assume $G$ is a classical group. Then

$$
d \Psi^{t}(\mathbf{C S}(\tilde{\delta}, \widetilde{G})) \subset \mathbf{C S}\left(G^{\ell}\right) .
$$

If $G=\operatorname{Sp}(2 n)$, we will prove equality in the next section. 


$$
\text { 14. } \mathbf{C S}(\operatorname{Spin}(n, n+1))=\mathbf{C S}(\tilde{\delta}, \widetilde{\operatorname{Sp}}(2 n, \mathbb{R}))
$$

In this section we use the theta correspondence to study the pseudospherical complementary series of $\widetilde{\operatorname{Sp}}(2 n, \mathbb{R})$. To better understand the limitations of this technique, first suppose that $\left(G_{1}, G_{1}^{\prime}\right)$ is an irreducible reductive dual pair in $\operatorname{Sp}(2 N, \mathbb{R})$. Write $\widetilde{G}_{1}$ and $\widetilde{G}_{1}^{\prime}$ for the respective preimages in $\widetilde{\operatorname{Sp}}(2 N, \mathbb{R})$. Then $\widetilde{G}_{1}$ and $\widetilde{G}_{1}^{\prime}$ are both linear except when (up to reordering) $G_{1}=\mathrm{O}(p, q)$ with $p+q$ odd and $G_{1}^{\prime}=\operatorname{Sp}(2 n, \mathbb{R})$. In this case $\widetilde{G}_{1}=\widetilde{\mathrm{O}}(p, q)$ is a linear cover of $\mathrm{O}(p, q)$, but $\widetilde{G}_{1}^{\prime}=\widetilde{\operatorname{Sp}}(2 n, \mathbb{R})$. Unfortunately, as mentioned above, $\widetilde{\operatorname{Sp}}(2 n, \mathbb{R})$ is the only nonlinear group arising this way, and the theta correspondence cannot be used to make conclusions about the other nonlinear groups in Conjecture 7.1.

With this in mind, fix a choice $\omega$ of one of the two oscillators for $\widetilde{\operatorname{Sp}}(2 N, \mathbb{R})$, and let $\operatorname{Irr}(\widetilde{\mathrm{O}}(p, q))$ and $\operatorname{Irr}(\widetilde{\mathrm{Sp}}(2 n, \mathbb{R}))$ denote the set of irreducible genuine representations of the indicated groups. The theta correspondence for the dual pair $(\mathrm{O}(p, q), \mathrm{Sp}(2 n, \mathbb{R}))$ may be interpreted as defining maps (depending on the choice of $\omega)$

$$
\begin{aligned}
& \theta_{n}^{(p, q)}: \operatorname{Irr}(\widetilde{\mathrm{Sp}}(2 n, \mathbb{R})) \longrightarrow \operatorname{Irr}(\widetilde{\mathrm{O}}(p, q)) \cup\{0\}, \\
& \theta_{(p, q)}^{n}: \operatorname{Irr}(\widetilde{\mathrm{O}}(p, q)) \longrightarrow \operatorname{Irr}(\widetilde{\mathrm{Sp}}(2 n, \mathbb{R})) \cup\{0\} .
\end{aligned}
$$

Here $\theta_{n}^{(p, q)}(\pi)=0$ if $\pi$ does not occur in the correspondence, and likewise for $\theta_{(p, q)}^{n}$. When restricted to those representations that do occur, these maps are inverses of each other. The basic reference for these facts is $[\mathrm{H} 2]$.

For our purposes, it will be convenient to work directly with $\mathrm{O}(p, q)$ instead of $\widetilde{\mathrm{O}}(p, q)$. Fix a genuine character $\xi$ of $\widetilde{\mathrm{O}}(p, q)$ as in $(1.2 \mathrm{~g})$ of $[\mathrm{AB}$. Then $\pi \mapsto \pi \otimes \xi$ is a bijection from the irreducible representations of $\mathrm{O}(p, q)$ to the irreducible genuine representations of $\widetilde{\mathrm{O}}(p, q)$. The inverse is given by tensoring with $\xi^{-1}$ : if $\pi$ is a genuine irreducible representation of $\widetilde{\mathrm{O}}(p, q)$, then $\pi \otimes \xi^{-1}$ is irreducible and factors to $\mathrm{O}(p, q)$. Define

$$
\bar{\theta}_{n}^{(p, q)}: \operatorname{Irr}(\widetilde{\operatorname{Sp}}(2 n, \mathbb{R})) \longrightarrow \operatorname{Irr}(\mathrm{O}(p, q)) \cup\{0\}
$$

by

Likewise define

$$
\bar{\theta}_{n}^{(p, q)}(\pi)=\theta_{n}^{(p, q)} \otimes \xi^{-1}
$$

$$
\bar{\theta}_{(p, q)}^{n}: \operatorname{Irr}(\mathrm{O}(p, q)) \longrightarrow \operatorname{Irr}(\widetilde{\mathrm{Sp}}(2 n, \mathbb{R})) \cup\{0\}
$$

by

$$
\bar{\theta}_{(p, q)}^{n}(\pi)=\theta_{(p, q)}^{n}(\pi \otimes \xi) .
$$

Now fix a pseudospherical representation $\tilde{\delta}$ for $\widetilde{\operatorname{Sp}}\left(2 n, \mathbb{R}\right.$ ). (The notation $\tilde{\delta}_{n}$ would be better, but we suppress the subscript.) If follows easily from the correspondence of joint harmonics that for any $M$ the oscillator defining $\bar{\theta}_{n}^{(M+1, M)}$ may be chosen so that $\bar{\theta}_{n}^{(M+1, M)}$ takes $\tilde{\delta}$-pseudospherical representations of $\widetilde{\operatorname{Sp}}(2 n, \mathbb{R})$ to spherical representations of $\mathrm{O}(M+1,1)$, and likewise that $\bar{\theta}_{(M+1, M)}^{n}$ takes spherical representations to pseudospherical ones. (The relevant calculation, together with an appropriate reference to the theory of joint harmonics, is recalled in Section 2, and especially Proposition 2.1, of $\mathrm{AB}$.) In the setting of Definition 1.1. a pseudospherical representation of $\widetilde{G}$ is characterized by its pseudospherical $K$-type and 
its infinitesimal character (see the comments after Definition 5.5). The following result is now a consequence of Theorem 5.1 of $[\mathrm{AB}]$.

Lemma 14.1. Retain the notation as above. Then

$$
\bar{\theta}_{(n+1, n)}^{n}\left(J_{\mathrm{O}(n+1, n)}(\nu)\right)=J_{\widetilde{\mathrm{Sp}}(2 n, \mathbb{R})}(\tilde{\delta}, \nu) ;
$$

here $\nu$ may be interpreted as a parameter for both $\mathrm{O}(n+1, n)$ and $\widetilde{\mathrm{Sp}}(2 n, \mathbb{R})$ by identifying the dual Cartan subspaces $\mathfrak{a}^{*}$ by the map $d \Psi^{t}$ as in Conjecture 7.1.

Theorem 14.2. The theta correspondence restricts to a bijection

$$
\bar{\theta}_{(n+1, n)}^{n}: \mathbf{C S}(\mathrm{O}(n+1, n)) \longrightarrow \mathbf{C S}(\tilde{\delta}, \widetilde{\mathrm{Sp}}(2 n, \mathbb{R})) .
$$

Since Lemma 4.20 shows that $\mathbf{C S}(\mathrm{O}(n+1,1))=\mathbf{C S}(\operatorname{Spin}(n+1, n))$, this establishes Conjecture 7.1 for $G=\operatorname{Sp}(2 n, \mathbb{R})$.

Proof. We need to introduce some auxiliary notation. Given an infinitesimal character $\nu$ for $\mathrm{O}(n+1, n)$, let $\left(\nu \mid \rho_{m}\right)$ denote the infinitesimal character for $\mathrm{O}(m+n+$ $1, m+n)$ obtained by augmenting the coordinates of $\nu$ by $(1 / 2,3 / 2, \ldots,(2 m-1) / 2)$. Let $J_{\mathrm{O}(m+n+1, m+n)}\left(\nu \mid \rho_{m}\right)$ denote the corresponding spherical representation of $\mathrm{O}(m+n+1, m+n)$. The following facts are crucial.

Fact $\mathbf{0}(\mathbf{a})$. If $J_{\mathrm{O}(n+1, n)}(\nu)$ is unitary, then each real coordinate of $\nu$ (in absolute value) is less than or equal to $(2 n-1) / 2$.

Fact $\mathbf{0}(\mathbf{b})$. If $J_{\widetilde{\mathrm{Sp}}(2 n, \mathbb{R})}(\tilde{\delta}, \nu)$ is unitary, then each real coordinate of $\nu$ (in absolute value) is less than or equal to $(2 n-1) / 2$.

Fact 1. If $m \geq n$, then $J_{\mathrm{O}(m+n+1, m+n)}\left(\nu \mid \rho_{m}\right)$ is unitary if and only if $J_{\mathrm{O}(n+1, n)}(\nu)$ is unitary.

Fact 2. If $m \geq n+2$, then $J_{\mathrm{O}(m+n+1, m+n)}\left(\nu \mid \rho_{m}\right)$ is a low-rank representation in the sense of [H1] (see also [Li2]). For the moment accept these facts; the proofs will be given below.

Consider the iterated lift

$$
\bar{\theta}_{n}^{(n+m+1, n+m)}\left(\bar{\theta}_{(n+1, n)}^{n}\left(J_{\mathrm{O}(n+1, n)}(\nu)\right)\right),
$$

a representation of the group $\mathrm{O}(n+m+1, n+m)$. Theorem 8.4 of $\mathrm{AB}$ ] (the induction principle) and the correspondence of joint harmonics imply

$$
\bar{\theta}_{n}^{(n+m+1, n+m)}\left(\bar{\theta}_{(n+1, n)}^{n}\left(J_{\mathrm{O}(n+1, n)}(\nu)\right)\right)=J_{\mathrm{O}(m+n+1, m+n)}\left(\nu \mid \rho_{m}\right) .
$$

Fix $\nu \in \mathbf{C S}(\mathrm{O}(n+1, n))$. We now show that $J_{\widetilde{\mathrm{Sp}}(2 n, \mathbb{R})}(\tilde{\delta}, \nu)$ is unitary, and thus establish one half of the theorem. Choose $m \geq n+2$. Consider $\pi:=$ $J_{\mathrm{O}(m+n+1, m+n)}\left(\nu \mid \rho_{m}\right)$. By Facts 1 and $2, \pi$ is a low-rank unitary representation of $\mathrm{O}(n+m+1, n+m)$. The main theorem of [Li2] thus asserts that there exists a character $\chi$ of $\mathrm{O}(n+m+1, n+m)$ and an irreducible unitary representation $\tau$ of some $\widetilde{\operatorname{Sp}}(2 l, \mathbb{R})$ with $l \leq(m+n) / 2$ such that

$$
\bar{\theta}_{l}^{(n+m+1, n+m)}(\tau)=\pi \otimes \chi .
$$

Our aim is to show that $l=n$ and that $\tau$ equals $J_{\widetilde{\mathrm{Sp}}(2 n, \mathbb{R})}(\tilde{\delta}, \nu)$. Since $\tau$ is unitary, this gives the desired unitarity of $J_{\widetilde{\mathrm{Sp}}(2 n, \mathbb{R})}(\tilde{\delta}, \nu)$.

Since $\pi$ is spherical, the correspondence of joint harmonics implies that the character $\chi$ in (14.4) must be trivial, and $\tau$ must be $\tilde{\delta}$-pseudospherical. 
Next we argue that the infinitesimal character correspondence dictates that $l=$ $n$. To see this note that if $\tau$ has infinitesimal character $\lambda$, then (see, for example, $[\mathrm{Pr}) \bar{\theta}_{l}^{(n+m+1, n+m)}(\tau)$ has infinitesimal character $\left(\lambda \mid \rho_{n+m-l}\right)$. (Here, as above, $\lambda$ is playing a dual role as an infinitesimal character for $\mathrm{O}$ and $\widetilde{\mathrm{Sp}}$; we will no longer remark on this explicitly.) So, as infinitesimal characters,

$$
\left(\lambda \mid \rho_{n+m-l}\right)=\left(\nu \mid \rho_{m}\right) .
$$

Using the Weyl group, we may assume each real coordinate above is positive, and we may also view the equality in (14.5) as an equality of $(n+m)$-tuples of complex numbers. First we prove that $l \geq n$. Suppose instead that $l<n$; so $n+m-l>m$, and the largest coordinate $\rho_{n+m-l}$ is strictly larger than the largest coordinate of $\rho_{m}$. Equation (14.5) thus implies that the largest coordinate of $\rho_{n+m-l}$ must be a coordinate of $\nu$. Since we have supposed $l<n$ and are assuming $m \geq n+2$ (in particular, $m \geq n$ ), the largest coordinate of $\rho_{n+m-l}$ is strictly larger than the largest coordinate of $\rho_{n}$, i.e., $(2 n-1) / 2$. But $\nu \in \mathbf{C S}(\mathrm{O}(n+1, n))$, and thus Fact 0 (a) gives a contradiction. So indeed $l \geq n$.

Next we suppose $l>n$ and derive a contradiction. If $l>n$, the largest coordinate of $\rho_{m}$ is greater than the largest coordinate of $\rho_{n+m-l}$ and Equation (14.5) implies that $\lambda$ must thus contain the largest coordinate of $\rho_{m}$. Recall that the theorem of [Li2 implies that $l \leq(m+n) / 2$; so the assumption $l>n$ implies $l<m$, and the largest coordinate of $\rho_{m}$ is strictly larger than the largest coordinate of $\rho_{l}$. So $\lambda$ contains a coordinate strictly larger than $(2 l-1) / 2$. But $\tau$ is pseudospherical, contradicting Fact $0(\mathrm{~b})$. Thus $l \leq n$.

The previous two paragraphs imply $l=n$ and that the infinitesimal character of $\tau$ equals $\nu$. Already we have seen that $\tau$ is pseudospherical, and hence (see after Definition 5.5) is characterized by its infinitesimal character. We conclude

$$
\tau=J_{\widetilde{\mathrm{Sp}}(2 n, \mathbb{R})}(\tilde{\delta}, \nu)
$$

as claimed. This proves one half of the theorem.

The other half of the theorem is easier and does not rely on Facts 0 or 2 . Fix $\nu \notin \mathbf{C S}(\mathrm{O}(n+1, n))$; we show that $\bar{\theta}_{(n+1, n)}^{n}\left(J_{\mathrm{O}(n+1, n)}(\nu)\right.$ is nonunitary. By Fact 1 ,

$$
\bar{\theta}_{n}^{(n+m+1, n+m)}\left(\bar{\theta}_{(n+1, n)}^{n}\left(J_{\mathrm{O}(n+1, n)}(\nu)\right)\right)=J_{\mathrm{O}(m+n+1, m+n)}\left(\nu \mid \rho_{m}\right)
$$

is nonunitary for $m \geq n$. But since $m \geq n$ (the so-called stable range case [Li1]), $\bar{\theta}_{n}^{(n+m+1, n+m)}$ takes unitary representations to unitary representations. So, indeed,

$$
\bar{\theta}_{(n+1, n)}^{n}\left(J_{\mathrm{O}(n+1, n)}(\nu)\right)=J_{\widetilde{\mathrm{Sp}}(2 n, \mathbb{R})}(\tilde{\delta}, \nu)
$$

cannot be unitary.

This completes the proof of Theorem 14.2 up to the proofs of Facts 0, 1, and 2. Fact 0 is standard and we begin there. After acting on $\nu$ by the Weyl group, we may suppose that the real coordinates of $\nu$ are the first $m$ (for some $m$ with $0 \leq m \leq n)$, and that

$$
\nu_{1} \geq \nu_{2} \geq \cdots \geq \nu_{m} \geq 0 .
$$

We will prove the contrapositive of Fact 0 : that if $\nu_{1} \geq n-1 / 2$, then the corresponding pseudospherical representation is not unitary. In fact we will prove nonunitarity under an even weaker condition. Here is the strengthened version of Fact $0(a)$. 
Lemma 14.6. Under the assumptions above on $\nu$, suppose that either

(1) $\nu_{m}>1 / 2$, or

(2) for some a with $1 \leq a \leq m-1$, we have $\nu_{a}-\nu_{a+1}>1$.

Then the spherical principal series representation $J_{\mathrm{O}(n+1, n)}(\nu)$ is not unitary.

Clearly the assumption that $\nu_{1}>n-1 / 2$ implies that at least one of the two conditions in the lemma is satisfied.

Proof. In the first case, set $a$ equal to $m$. Define

$$
\nu_{t}=(t, \ldots, t, 0, \ldots, 0)+\nu,
$$

with the first $a$ coordinates equal to $t$. We consider the family of spherical representations $J_{\mathrm{O}(n+1, n)}\left(\nu_{t}\right)$ for $t \geq 0$. What we are trying to show is that $J_{\mathrm{O}(n+1, n)}\left(\nu_{0}\right)=$ $J_{\mathrm{O}(n+1, n)}(\nu)$ is not unitary. If this representation admits no nonzero invariant Hermitian form, we are done; so we may assume that such a form exists and normalize it to be positive on the $K$-fixed vector. Knapp's "formal symmetry condition" for the existence of such a form is inherited by all $\nu_{t}$; so we may endow all the representations $J_{\mathrm{O}(n+1, n)}\left(\nu_{t}\right)$ (for $t \geq 0$ ) with nondegenerate invariant Hermitian forms positive on the spherical vectors. We will investigate this family of forms on the "p $K$-type": the representation of $\mathrm{O}(n+1) \times \mathrm{O}(n)$ on $\mathbb{C}^{n+1} \otimes \mathbb{C}^{n}$, which we will call $\mu_{1}$. This is a petite $K$-type, corresponding to $k=1$ in the first case of Lemma 12.7 It is the adjoint representation of $K$ on $\mathfrak{p}_{\mathbb{C}}$ in the Cartan decomposition $\mathfrak{g}=\mathfrak{k}+\mathfrak{p}$. According to Lemma 12.7, the representation of $W$ on the space $V_{\mu_{1}}(\mathbf{1})$ of $M$-fixed vectors is $\sigma_{1}=(n-1) \times(1)$, which is the reflection representation of $W$. According to Corollary 13.19, the Hermitian form for $J_{\mathrm{O}(n+1, n)}\left(\nu_{t}\right)$ on the $K$-type $\mu_{1}$ is given by the matrix $A\left(\sigma_{1}, \nu_{t}\right)$. What we will show is that for all $t \geq 0, A\left(\sigma_{1}, \nu_{t}\right)$ fails to be positive definite; this will prove in particular that $J_{\mathrm{O}(n+1, n)}(\nu)$ is not unitary.

The proof that $A\left(\sigma_{1}, \nu_{t}\right)$ fails to be positive definite has two steps. First, we will show that the signature of $A\left(\sigma, \nu_{t}\right)$ is constant for all $t \geq 0$, for any Weyl group representation $\sigma$. It therefore suffices to prove that $A\left(\sigma_{1}, \nu_{t}\right)$ is indefinite for large $t$. This we will do by inspection of the Casimir operator for $G$.

For the first step, let

$$
L=\operatorname{GL}(a, \mathbb{R}) \times O(n-a+1, n-a)
$$

be the maximal Levi subgroup of $G$ defined by the weight $(1, \ldots, 1,0, \ldots, 0)$ (with $a$ terms equal to one). Let $P=L U$ be the corresponding standard parabolic subgroup. The roots in $U$ are

$$
e_{i} \pm e_{j}, \quad e_{i} \quad(1 \leq i \leq a, a+1 \leq j \leq n) .
$$

What follows from the hypothesis on $a$ in the lemma is that

$$
\left\langle\nu, \alpha^{\vee}\right\rangle \text { is strictly greater than } 1 \text { or nonreal }
$$

for all roots $\alpha$ in $U$. (The first possibility occurs if $j$ is less than or equal to $m$ or absent, and the second if $j$ is greater than $m$.)

In the factorization 13.3$)(\mathrm{b})$, each factor is an invertible operator $\left\langle\nu, \alpha^{\vee}\right\rangle= \pm 1$. This is a consequence of the definition of the factors in Proposition $(13.2)$ (a). Now it follows from (14.7) that the rank of $A(\sigma, t)$ is independent of $t$ (for $t \geq 0$ ); in fact it is equal to the rank of the corresponding operator for the Weyl group of $L$. Because the positive and negative parts of the signature are semicontinuous in 
$t$, the constancy of the rank (equal to the sum of the positive and negative parts) implies that the signature is constant as well.

It remains to show that $A\left(\sigma_{1}, t\right)$ is not positive definite for large positive $t$. For this we can invoke [Sal, Lemma 6.3]; since the argument in our case is very simple, we will give a proof. It is most convenient to work with the group representation. Let $v_{0}(t)$ be a nonzero $K$-fixed unit vector in $J_{\mathrm{O}(n+1, n)}\left(\nu_{t}\right)$. We use the invariant bilinear form on $\mathfrak{g}$ extending our chosen inner product on $\mathfrak{a}$, and let $\Omega$ be the corresponding Casimir operator. In a representation $\pi$ with infinitesimal character $\lambda$, we have

$$
\pi(\Omega)=\langle\lambda, \lambda\rangle-\langle\rho, \rho\rangle
$$

(with $\rho$ the half sum of a set of positive roots). In particular, $\Omega$ must act on $J\left(\nu_{t}\right)$ by the scalar

$$
\left\langle\nu_{t}, \nu_{t}\right\rangle-\langle\rho, \rho\rangle \text {. }
$$

This is a quadratic polynomial in $t$ with leading term $a t^{2}$, so it certainly has positive real part for $t$ sufficiently large.

Now assume that the invariant Hermitian form $\langle\cdot, \cdot\rangle$ is positive on the $\mathfrak{p} K$ type $\mu_{1}$. We will compute the Casimir eigenvalue in a different way and get a contradiction. Choose an orthonormal basis $\left\{X_{i}\right\}$ for $\mathfrak{p}$, and an orthogonal basis $\left\{Y_{j}\right\}$ of $\mathfrak{k}$ consisting of vectors of length -1 . This is possible since the invariant form on $\mathfrak{g}$ is positive on $\mathfrak{p}$ and negative on $\mathfrak{k}$. Then

$$
\Omega=\sum_{i} X_{i}^{2}-\sum_{j} Y_{j}^{2} .
$$

Since $v_{0}(t)$ is a unit vector in $J\left(\nu_{t}\right)$, the Casimir eigenvalue is

$$
\left\langle\Omega \cdot v_{0}(t), v_{0}(t)\right\rangle_{t}=\sum_{i}\left\langle X_{i}^{2} \cdot v_{0}(t), v_{0}(t)\right\rangle_{t}+\sum_{j}\left\langle Y_{j}^{2} \cdot v_{0}(t), v_{0}(t)\right\rangle_{t} .
$$

Since $v_{0}(t)$ is $K$-fixed, all terms in the second sum are zero. In the first sum, we can use the invariance of the inner product to move one $X_{i}$ to the right with a sign change, getting

$$
\sum_{i}-\left\langle X_{i} \cdot v_{0}(t), X_{i} \cdot v_{0}(t)\right\rangle_{t}
$$

Now $v_{0}(t)$ is $K$-fixed and the $X_{i}$ transform by the representation $\mu_{1}$; so all of the vectors $X_{i} \cdot v_{0}(t)$ must belong to the $\mu_{1} K$-type of $J\left(\nu_{t}\right)$. By hypothesis the invariant Hermitian form is positive on this $K$-type, so each vector $X_{i} \cdot v_{0}(t)$ has nonnegative length. The conclusion is that the eigenvalue of the Casimir must be nonpositive, contradicting our earlier calculation that the eigenvalue has positive real part for large $t$. This contradiction proves that the invariant form must be nonpositive on $\mu_{1}$ for large $t$.

Here is the strengthened version of Fact $0(b)$.

Lemma 14.8. Suppose that the real coordinates of $\nu$ are the first $m$ and that

$$
\nu_{1} \geq \nu_{2} \geq \cdots \geq \nu_{m} \geq 0 \text {. }
$$

Assume that either

(1) $\nu_{m}>1 / 2$, or

(2) for some a with $1 \leq a \leq m-1$, we have $\nu_{a}-\nu_{a+1}>1$.

Then the pseudospherical principal series representation $J_{\widetilde{\mathrm{Sp}}(2 n, \mathbb{R})}(\nu)$ is not unitary. 
Proof. We follow as closely as possible the proof of Lemma 14.6. We define $\nu_{t}$ exactly as in the preceding proof and consider the family of pseudospherical representations $J_{\widetilde{\mathrm{Sp}}(2 n, \mathbb{R})}\left(\nu_{t}\right)$. As before we may assume that $J_{\widetilde{\mathrm{Sp}}(2 n, \mathbb{R})}\left(\nu_{0}\right)$ admits an invariant Hermitian form. Suppose for definiteness that $\tilde{\delta}=\tilde{\delta}_{+}$, so that all these representations contain the $\widetilde{K}$-type $\operatorname{det}^{1 / 2}$. We normalize the forms $\langle\cdot, \cdot\rangle_{t}$ to be positive on the pseudospherical $\widetilde{K}$-type $\operatorname{det}^{1 / 2}$. We investigate the forms on the $\widetilde{K}$-type $\widetilde{\mu}_{1}$ of highest weight

$$
(1 / 2, \ldots, 1 / 2,-3 / 2) \text {. }
$$

This is a petite $\widetilde{K}$-type, corresponding to $k=1$ in the first case of Lemma 12.5. The corresponding representation of $W$ is again $\sigma_{1}=(n-1) \times(1)$, the reflection representation. According to Corollary 13.19, the Hermitian form for $J_{\widetilde{\mathrm{Sp}}(2 n, \mathbb{R})}\left(\nu_{t}\right)$ on the $\widetilde{K}$-type $\widetilde{\mu}_{1}$ is given by the matrix $\widetilde{A}\left(\sigma_{1}, \nu_{t}\right)$. According to Lemma 13.7 this is equal to $A\left(\sigma_{1}, \nu_{t}\right)$. The fact that this matrix is not positive definite was already proved in Lemma 14.6, so we are done.

There is a small subtlety here. The proof given for failure of positivity of the matrix $A\left(\sigma_{1}, \nu_{t}\right)$ in Lemma 14.6 does not trivially work in the setting of Lemma 14.8. The main difficulty is that the tensor product of $\operatorname{det}^{1 / 2}$ with the representation $\mathfrak{p}_{\mathbb{C}}$ of $\widetilde{K}$ contains not only $\widetilde{\mu}_{1}$, but also the nonpetite representation of $\widetilde{K}$ of highest weight $(5 / 2,1 / 2, \ldots, 1 / 2)$. (One can deal with this difficulty with a little more care in the argument.) But the point is that we do not need a new representationtheoretic argument to prove that $A\left(\sigma_{1}, \nu_{t}\right)$ is not positive, since it is exactly the same matrix appearing in the linear case.

Facts 1 and 2 are consequences of the shape of the main results of [Ba1]; here we simply supply a few details.

We begin with a brief summary of Ba1]. For notational ease, we assume $G=$ $\mathrm{O}(n+1, n), \mathrm{O}(n, n)$ or $\mathrm{Sp}(2 n, R)$. (Of course, the first case is the one relevant for Facts 1 and 2, but it is conceptually easier to work in this slightly more general setting.) It is convenient also to use a possibly disconnected version of the complex dual group, writing

$$
G_{\mathbb{C}}^{\vee}= \begin{cases}\mathrm{Sp}(2 n, \mathbb{C}) & \text { if } G=\mathrm{O}(n+1, n), \\ \mathrm{O}(2 n, \mathbb{C}) & \text { if } G=\mathrm{O}(n, n), \text { and } \\ \mathrm{O}(2 n+1, \mathbb{C}) & \text { if } G=\mathrm{Sp}(2 n, \mathbb{R}) .\end{cases}
$$

As usual, the duality relationship between $\mathfrak{g}$ and $\mathfrak{g}_{\mathbb{C}}^{\vee}$ identifies $\mathfrak{a}_{\mathbb{C}}^{*}$ as a Cartan subalgebra of $\mathfrak{g}_{\mathbb{C}}^{\vee}$, in a way that is canonical up to the action of $W$. In the usual coordinates for these classical groups, the identification comes from the identification of each space with $\mathbb{C}^{n}$.

To each spherical representation $J(\nu)$ of $G$, Ba1 first attaches a nilpotent orbit $\mathcal{O}^{\vee}$ in the complex dual Lie algebra $\mathfrak{g}_{\mathbb{C}}^{\vee}$. Explicitly, $\mathcal{O}^{\vee}$ is the unique dense orbit in the $G_{\mathbb{C}}^{\vee}$ saturation of the 1-eigenspace of $\operatorname{ad}(\nu)$ on $\mathfrak{g}_{\mathbb{C}}^{\vee}$. Let $p^{\vee}=p\left(\mathcal{O}^{\vee}\right)=\left\{p_{1}^{\vee} \geq\right.$ $\left.p_{2}^{\vee} \geq \cdots\right\}$ denote the partition obtained from the Jordan normal form of an element of $\mathcal{O}^{\vee}$. We break the partition into two pieces $p^{\vee}=p_{\text {unip }}^{\vee} \cup p_{\mathrm{GL}}^{\vee}$ as follows:

$$
p_{\text {unip }}^{\vee}=\text { one copy of each part of } p^{\vee} \text { having odd multiplicity, }
$$

and $p_{\mathrm{GL}}^{\vee}$ consists of the remaining parts (each therefore appearing with even multiplicity). The partition $p_{\text {unip }}^{\vee}$ parametrizes a distinguished nilpotent orbit $\mathcal{O}_{\text {unip }}^{\vee}$ in 
a classical Lie algebra $\mathfrak{g}(k)_{\mathbb{C}}^{\vee}$ of a complex Lie algebra $\mathfrak{g}(k)_{\mathbb{C}}$ of rank $k$ and the same type as $\mathfrak{g}_{\mathbb{C}}^{\vee}$. (For classical groups outside of Type $A$, an orbit is distinguished if and only if the partition corresponding to its Jordan normal form has no repeated entries.) The partition $p_{\mathrm{GL}}^{\vee}$ may be written

$$
p_{\mathrm{GL}}^{\vee}=\left\{n_{1}, n_{1} \geq n_{2}, n_{2} \geq \cdots \geq n_{r}, n_{r}\right\} .
$$

We now define a Levi subgroup

$$
L=G(k) \times \mathrm{GL}\left(n_{1}, \mathbb{R}\right) \times \cdots \times \mathrm{GL}\left(n_{r}, \mathbb{R}\right) \subset G ;
$$

here $G(k)$ is a split classical group of rank $k$ and the same type as $G$. Let $\pi_{\text {unip }}$ denote the spherical representation of $G(k)$ whose infinitesimal character $\nu_{\text {unip }}$ is one-half the semisimple element of a Jacobson-Morozov triple for $\mathcal{O}_{\text {unip }}^{\vee}$. (A distinguished nilpotent orbit is even, and $\nu_{\text {unip }}$ is therefore integral.) In the course of describing how to calculate the partition $p^{\vee}$ from $\nu$, Ba1 defines characters $\chi_{i}$ of each $\operatorname{GL}\left(n_{i}\right)$ factor of $L$. These characters are chosen so that $J(\nu)$ is a composition factor of $\operatorname{Ind}_{L}^{G}\left(\pi_{\text {unip }} \otimes \chi_{1} \otimes \cdots \otimes \chi_{r}\right)$. (Here we really mean parabolic induction, of course, but we are being a little sloppy and not specifying the nilradical.)

Write $Z\left(\mathcal{O}^{\vee}\right)_{\mathbb{C}}^{\vee}$ for the centralizer in $G_{\mathbb{C}}^{\vee}$ of a Jacobson-Morozov triple for $\mathcal{O}^{\vee}$. Because $G^{\vee}$ is a symplectic or orthogonal group, this centralizer is a product of symplectic and orthogonal groups $(\underline{\mathrm{CMc}})$ :

$$
Z\left(\mathcal{O}^{\vee}\right)_{\mathbb{C}}^{\vee}=\prod_{i} \mathrm{Sp}\left(2 a_{i}, \mathbb{C}\right) \times \prod_{j} O\left(2 b_{j}, \mathbb{C}\right) \times \prod_{k} \mathrm{O}\left(2 c_{k}+1, \mathbb{C}\right) .
$$

(The numbers $2 a_{i}, 2 b_{j}$, and $2 c_{k}+1$ are the multiplicities of parts of $p^{\vee}$.) Define $Z\left(\mathcal{O}^{\vee}\right)$ to be the split real form of the dual group,

$$
Z\left(\mathcal{O}^{\vee}\right)=\prod_{i} \mathrm{O}\left(a_{i}+1, a_{i}\right) \times \prod_{j} O\left(b_{j}, b_{j}\right) \times \prod_{k} \mathrm{Sp}\left(2 c_{k}, \mathbb{R}\right) .
$$

From the characters $\chi_{i}$, Ba1] defines an infinitesimal character $\nu_{Z}$ for $Z\left(\mathcal{O}^{\vee}\right)$. Since $Z\left(\mathcal{O}^{\vee}\right)$ is a product of orthogonal and symplectic groups, one may run the algorithms just described and extract from $\nu_{Z}$ a Levi subgroup, an orbit in the dual, et cetera, with $Z\left(\mathcal{O}^{\vee}\right)$ now playing the role of $G$ in the previous paragraph. Let $\mathcal{O}_{Z}^{\vee}$ denote the nilpotent orbit in the dual $\mathfrak{z}\left(\mathcal{O}^{\vee}\right)_{\mathbb{C}}^{\vee}$ described by the procedure above. One version of the main theorem of [Ba1] is that

$J_{G}(\nu)$ is unitary if and only if $J_{Z\left(\mathcal{O}^{\vee}\right)}\left(\nu_{Z}\right)$ is unitary and $\mathcal{O}_{Z}^{\vee}$ is the zero orbit.

We will deduce Facts 1 and 2 by examining how the data described above change when we pass from $J_{\mathrm{O}(n+1, n)}(\nu)$ to $J_{\mathrm{O}(n+m+1, n+m)}\left(\nu \mid \rho_{m}\right)$ for $m \geq n$. We retain the notation above for the data attached to $\nu$ and add primes for the data attached to $\nu^{\prime}=\left(\nu \mid \rho_{m}\right)$. It is not difficult to show that

$$
\left(p^{\vee}\right)^{\prime}=p^{\vee} \text { augmented by the part } 2 m \text {. }
$$

Since $m \geq n$ by assumption (and this assumption is crucial), the part $2 m$ is larger than any in $p_{\text {unip }}^{\vee}$ and the partition $\left(p_{\text {unip }}^{\vee}\right)^{\prime}$ consists of distinct parts and thus parametrizes a distinguished orbit unless both $m=n$ and $p^{\vee}=p_{\text {unip }}^{\vee}=\{2 n\}$. We return to this situation below; for now assume we are not in this case. It follows that if $L=G(k) \times \operatorname{GL}\left(n_{1}\right) \times \cdots \times \mathrm{GL}\left(n_{r}\right)$ is the Levi factor for $\nu$, the $L^{\prime}$ for $\nu^{\prime}$ is simply $G(k+m) \times \mathrm{GL}\left(n_{1}\right) \times \cdots \times \mathrm{GL}\left(n_{r}\right)$. The construction in Ba1 shows that the characters $\chi_{i}^{\prime}$ coincide with the $\chi_{i}$. Because there is a single distinct extra part 
in $\left(p^{\vee}\right)^{\prime}$, the centralizer subgroup $Z^{\prime}\left(\left(\mathcal{O}^{\vee}\right)^{\prime}\right)$ is $\mathrm{O}(1,0) \times Z\left(\mathcal{O}^{\vee}\right)$; the infinitesimal character $\nu_{Z}^{\prime}$ coincides with $\nu_{Z}$. Thus determining the unitarity of $J_{\mathrm{O}(n+1, n)}(\nu)$ and determining the unitarity of $J_{\mathrm{O}(n+m+1, n+m)}\left(\nu \mid \rho_{m}\right)$ reduces via Equation (14.10) to determining the unitarity of the same representation of $Z\left(\mathcal{O}^{\vee}\right)$. (The extra factor of $\mathrm{O}(1,0)=\mathbb{Z} / 2 \mathbb{Z}$ has no effect on unitarity.) This establishes Fact 1.

Return to the case where $m=n$ and $p^{\vee}=p_{\text {unip }}^{\vee}=\{2 n\}$. Then $J_{\mathrm{O}(n+1, n)}(\nu)$ is the trivial representation and of course unitary. On the other hand, $J_{\mathrm{O}(2 n+1,2 n)}\left(\nu \mid \rho_{n}\right)$ is clearly contained in the representation unitarily induced from the trivial character on the Siegel $\operatorname{GL}(2 n, \mathbb{R})$ Levi factor, and hence is unitary. So Fact 1 holds in this case as well.

We now turn to Fact 2, beginning with some preliminaries about low rank representations. Given a representation $\pi$, let $\operatorname{WF}(\pi)$ denote Howe's wavefront set of $\pi$; for a definition and some basic properties, see [H0] and [BV0]. Define the rank of a set $S$ of nilpotent matrices in a classical Lie algebra to be $\max \{\operatorname{rank}(X) \mid X \in S\}$; here $\operatorname{rank}(X)$ is the rank of $X$ as a matrix. Set

$$
r(N+1, N)= \begin{cases}N-1 & \text { if } N \text { is odd } \\ N & \text { if } N \text { is even }\end{cases}
$$

A representation $\pi$ of $\mathrm{O}(N+1, N)$ is said to be of low rank if

$$
\operatorname{rank}(\mathrm{WF}(\pi))<r(N+1, N) .
$$

(This is not the original definition of rank given by Howe [H1]. That this definition coincides with Howe's is an unpublished theorem of Howe (one direction) and Li (the other); see [Li3]. The corresponding theorem for the symplectic group is stated and proved in [Li4, where it is stated that analogous theorems hold for other classical groups. That the formulation given here is the right one for orthogonal groups follows from [Li2, (14)].) In terms of the partition classification of nilpotent orbits, the rank of a nilpotent matrix is equal to the dimension of the vector space minus the number of parts of the partition; that is, the dimension minus the length of the first column. We conclude that a representation $\pi$ of $\mathrm{O}(N+1, N)$ has low rank if and only if

$$
\text { there is a column in a partition for } \operatorname{WF}(\pi) \text { of length } \geq \begin{cases}N+3 & N \text { odd; } \\ N+2 & N \text { even. }\end{cases}
$$

In order to establish Fact 2, we must therefore show that

there is a column in $\mathrm{WF}\left(J_{\mathrm{O}(m+n+1, m+n)}\left(\nu \mid \rho_{m}\right)\right)$ of length at least $m+n+3$.

Consider the Levi subgroup

$$
L^{\prime}=\mathrm{O}(m+1, m) \times \mathrm{GL}(1, \mathbb{R})^{n} \subset \mathrm{O}(m+n+1, m+n) .
$$

Because $\rho_{m}$ is the infinitesimal character of the trivial representation of $\mathrm{O}(m+1, m)$, we have

$$
J_{\mathrm{O}(m+n+1, m+n)}\left(\nu \mid \rho_{m}\right) \text { is a comp. factor of } \operatorname{Ind}_{L^{\prime}}^{\mathrm{O}(m+n+1, m+n)} \text { (one-diml. char.). }
$$

It follows that

$$
\mathrm{WF}\left(J_{\mathrm{O}(m+n+1, m+n)}\left(\nu \mid \rho_{m}\right)\right) \subset \text { closure of Richardson orbit for } L^{\prime} .
$$


Because the length of the longest column in the partition can only increase in an orbit closure, what we want to show comes down to

there is a column in the Richardson orbit for $L^{\prime}$ of length at least $m+n+3$.

This Richardson orbit is easy to compute, and we find that the corresponding partition is $(2 n+1)(1)^{2 m}$. Its longest column has length $2 m+1$, which is at least $m+n+3$ since $m \geq n+2$. This establishes Fact 2 .

Corollary 14.12. The $\widetilde{K}$ types appearing in the proof of Theorem 13.24 detect pseudospherical unitarity for $\widetilde{\mathrm{Sp}}(2 n, \mathbb{R})$ (Definition 13.11).

Proof. This follows from Theorem 14.2 and Proposition 13.21 .

Remark 14.13. Half of Theorem 14.2 follows from Theorem 13.24 this corresponds to the part of the argument that we gave second, which used only Fact 1 . Because the spherical unitary dual of a split real group is the "same" as the spherical unitary dual of split $p$-adic groups ([Ba1]) and since the results of [Li2] are valid over any local field of characteristic not 2, the proof of Theorem 14.2 (with suitable but essentially obvious modifications) is valid for $p$-adic orthogonal and symplectic groups (of characteristic not 2). The resulting correspondence of unitary representations should be a special case of the conjecture discussed in Remark 7.3 .

\section{REFERENCES}

[A] J. Adams, Nonlinear covers of real groups, Int. Math. Res. Not. 75 (2004), 4031-4047. MR2112326 (2006c:20096)

[AB] J. Adams, D. Barbasch, Genuine representations of the metaplectic group, Compositio Math. 113 (1998), 23-66. MR1638210 (99h:22013)

[BV0] D. Barbasch, D. A. Vogan, Jr., The local structure of characters, J. Func. Anal. 37 (1980), 27-55. MR0576644 (82e:22024)

[Ba1] D. Barbasch, Spherical unitary dual of split classical groups, preprint. Available at http://www. math. cornell. edu/ barbasch/.

[Ba2] D. Barbasch, Relevant and petite $K$-types for split groups, Functional analysis VIII, 35-71, Various Publ. Ser. (Aarhus), 47, Aarhus Univ., Aarhus, 2004. MR2127163 (2006d:22020)

[Bo] N. Bourbaki, Groupes et Algèbres de Lie, Chapitres 4, 5, et 6, Masson, Paris, 1981. MR0647314 (83g:17001)

[CMc] D. Collingwood, W. M. McGovern, Nilpotent orbits in semisimple Lie algebras, Van Nostrand Reinhold Mathematics Series, New York, 1993. MR.1251060 (94j:17001)

[Ci] D. Ciubotaru, The unitary $\mathbb{I}$-spherical dual for split $p$-adic groups of type $F_{4}$, Represent. Theory 9 (2005), 94-137. MR2123126 (2005k:22022)

[CR] C. W. Curtis and I. Reiner, Methods of representation theory. Vol. I, John Wiley \& Sons Inc., New York, 1981. MR0632548 (82i:20001)

[H0] R. Howe, Wave front sets of representations of Lie groups, in Automorphic Forms, Representation Theory, and Arithmetic, Tata Inst. Fund. Res. Studies in Math., volume 10. Tata Institute for Fundamental Research, Bombay, 1981. MR0633659 (83c:22014)

[H1] R. Howe, On a notion of rank for unitary representations of the classical groups, in Harmonic analysis and group representations, 223-331, Liguori (Naples) 1982. MR0777342 (86j:22016)

[H2] R. Howe, Transcending classical invariant theory, J. Amer. Math. Soc. 2 (1989), 535-552. MR $0985172(90 \mathrm{k}: 22016)$

[Hu1] J.-S. Huang, The unitary dual of the universal covering group of GL(n, R $)$, Duke Math. J. 61 (1990), 705-745. MR:1084456 (92f:22026)

[Hu2] J.-S. Huang, Metaplectic correspondences and unitary representations, Compositio Math. 80 (1991), 309-322. MR1134258 (93d:22021)

[Kn] A. W. Knapp, Representation Theory of Semisimple Groups: An Overview Based on Examples, Princeton Mathematical Series 36, Princeton University Press, Princeton, New Jersey, 1986. MR0855239 (87j:22022) 
[Li1] J.-S. Li, Singular unitary representations of classical groups, Invent. Math. 97 (1989), 237-255. MR 1001840 (90h:22021)

[Li2] J.-S. Li, On the classification of irreducible low rank unitary representations of classical groups. Compositio Math. 71 (1989), 29-48. MR1008803 (90k:22027)

[Li3] J.-S. Li, Unipotent representations attached to small nilpotent orbits, unpublished lecture notes from a 1997 AMS conference in Seattle, available at http://www.math.umd. edu/ jda/seattle_proceedings/.

[Li4] J.-S. Li, On the singular rank of a representation, Proc. Amer. Math. Soc. 106 (1989), 567-571. MR0961413 (89k:22029)

[Ma] H. Matsumoto, Sur les sous-groupes arithmétiques des groupes semi-simples déployés, Ann. Sci. Ecole Norm. Sup. 2 (1969), 1-62. MR0240214 (39:1566)

[Pr] T. Przebinda, The duality correspondence of infinitesimal characters, Colloq. Math. 70 (1996), 93-102. MR:1373285 (96m:22034)

[Sal] S. Salamanca, The unitary dual of some classical Lie groups, Compositio Math. 68 (1988), 251-303. MR0971329 (89m:22018)

[Sa] G. Savin, Local Shimura correspondence, Math. Ann. 280 (1988), 185-190. MR0929534 (89h:22018)

[Sa2] G. Savin, On unramified representations of covering groups, J. Reine Angew. Math. 566 (2004), 111-134. MR2039325 (2005a:22014)

[Sc] W. Schmid, "On the characters of the discrete series (the Hermitian symmetric case)", Invent. Math. 30 (1975), 47-144. MR0396854 (53:714)

[St] R. Steinberg, Générateurs, relations, et revêtements de groupes algébriques, 113-127 in Colloq. Théorie des Groupes Algébriques (Bruxelles, 1962). Librairie Universitaire, Louvain, 1962. MR0153677 (27:3638)

[V0] D. A. Vogan, Jr., The algebraic structure of the representations of semisimple Lie groups I, Ann. Math. 109 (1979), 1-60. MR.0519352 (81j:22020)

[V2] D. A. Vogan, Jr., The unitary dual of GL $(n)$ over an Archimedean field, Invent. Math. 83 (1986), 449-505. MR0827363 (87i:22042)

[V3] D. A. Vogan, Jr., The unitary dual of $\mathrm{G}_{2}$, Invent. Math. 116 (1994), 677-791. MR.1253210 (95b:22037)

[V4] D. A. Vogan, Jr., Representations of Real Reductive Lie Groups, Progress in Mathematics 15, Birkhäuser, Boston, 1981. MR0632407 (83c:22022)

[W] N. R. Wallach, Real Reductive Groups II, Academic Press, San Diego, 1992. MR.1170566 (93m:22018)

Department of Mathematics, University of Maryland, College Park, Maryland 20742

E-mail address: jda@math.umd.edu

Department of Mathematics, Cornell University, Ithaca, New York 14853

E-mail address: barbasch@math.cornell.edu

Department of Mathematics, Western Michigan University, Kalamazoo, Michigan 49008

E-mail address: paula@wmich.edu

Department of Mathematics, University of Utah, Salt lake City, Utah 84112

E-mail address: ptrapa@math.utah.edu

Department of Mathematics, Massachusetts institute of Technology, Cambridge, MassachusetTs, 02138

E-mail address: dav@math.mit.edu 